\title{
ESTUDO DO ÍNDICE DE REFRAÇÃO NÃO-LINEAR EM CRISTAIS E VIDROS FLUORETOS DOPADOS
}

Acácio A. de Castro Andrade

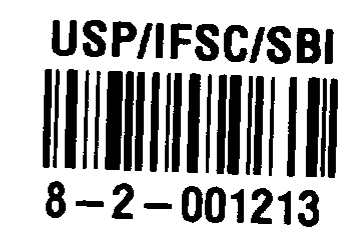

Dissertação apresentada ao Instituto de Física de são Carlos, da Universidade de São Paulo, para obtenção do título de Mestre em Ciências: Física Aplicada.

Orientador: Prof. Dr. Tomaz Catunda

São Carlos

1998.

$10 \ldots$ SEFVCO DE PINIOTECA E 


Andrade, Acácio A. de Castro
Estudo do índice de refração não-linear em cristais e
vidros fluoretos dopados / Acácio A. de Castro
Andrade - São Carlos, 1998.
$99 \mathrm{p}$
Dissertação (Mestrado) - Instituto de Física de São
Carlos, 1998.
Orientador: Prof: Dr: Tomaz Catunda
1. Índice de refração não-linear. 2. Sólidos Dopados.
I. Título


MEMBROS DA COMISSÃO JULGADORA DA DISSERTAÇÃO DE MESTRADO DE ACÁCIO APARECIDO DE CASTRO ANDRADE APRESENTADA AO INSTITUTO DE FÍSICA DE SÃO CARLOS, DA UNIVERSIDADE DE SÃO PAULO, EM 08 DE MAIO DE 1998.

COMISSÃO JULGADORA:

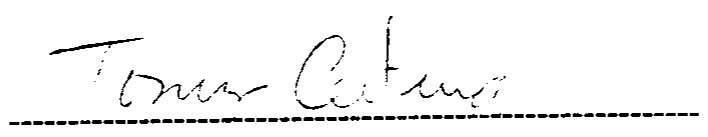

Prof. Dr. Tomaz Catunda/IFSC-USP

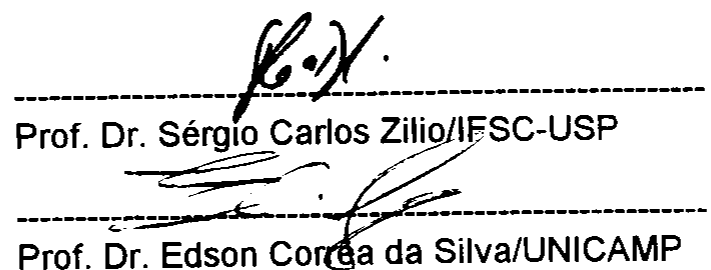


A minha esposa Rosimeire pela compreensão e carinho nas horas dificeis. 


\section{AGRADECIMENTOS}

- Ao Prof. Tomaz Catunda pela orientação, apoio, incentivo e amizade durante o desenvolvimento deste trabalho.

- A minha família, em especial a minha mãe Cláire e a minha madrinha Leonora, que me apoiaram desde o início de minha carreira.

- Ao Prof. Mauro L. Baesso pela ajuda nas medidas de Lente Térmica e pela amizade.

- Aos colegas Eik Tenório, Sandro M. Lima e Juraci Sampaio pelas discussões a cerca dos resultados.

- Ao técnico do laboratório André Romero pela ajuda na parte computacional.

- E a todos os funcionário do Instituto de Física, que de uma maneira ou outra contribuíram para a realização deste trabalho.

- A FAPESP pelo suporte financeiro. 
SUMÁRIO

CAPÍTULO 1 INTRODUÇÃo 1

CAPÍTULO 2 ESPECTROSCOPIA EM SÓLIDOS DOPADOS . .4

2.1. SÍNTESE DOS VIDROS 5

2.2. CARACTERÍSTICAS ESPECTROSCÓPICAS DE SÓLIDOS DOPADOS COM ND ${ }^{+3} \ldots \ldots .7$

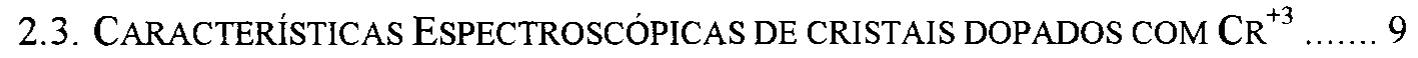

2.4. INVESTIGAÇÃO DA ORIGEM DA NÃO-LINEARIDADE EM SÓLIDOS DOPADOS ..... 10

2.5. EQUAÇÕES DE TAXA 12

CAPÍTULO 3 TÉCNICAS DE Z-SCAN E LENTE TÉRMICA 19

3.1. TÉCNICA DE Z-SCAN 20

3.2. A INTEGRAL DE DIFRAÇÃO DE KIRCHHOFF 23

3.3. TRATAMENTO TEÓRICO DA TÉCNICA Z-SCAN 29

3.4. ABSORÇÃO NÃO LINEAR . 34

3.5. Z-SCAN NO CASO DE NÃO-LINEARIDADE SATURȦVEL 38

3.6. TÉCNICA DE LENTE TÉRMICA 40

CAPÍTULO 4 RESULTADOS EXPERIMENTAIS .46

4. 1. MEdidAs DE LENTE TÉRMICA 47

4.1.1. Montagem experimental. 47

4.1.2. Resultados . 51

4.2. MEDIDAS DE Z-SCAN.... 53

4.2.1. Montagem Experimental. 53

4.2.2. Resultados em cristais dopados com $\mathrm{Cr}^{-3}$ 56 


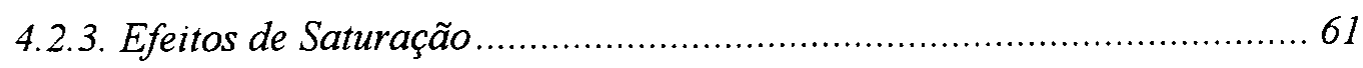

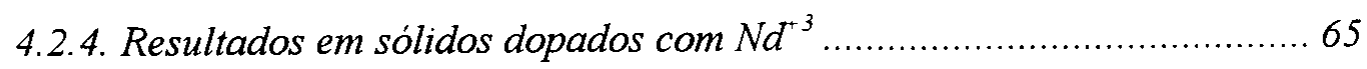

CAPÍTULO 5 ESTUdO DO EFEITO TÉRMICO E ELETRÔNICO ................ 70

5.1. EFEITO TÉRMICO E ELETRÔNICO SIMULTÂNEOS ................................... 71

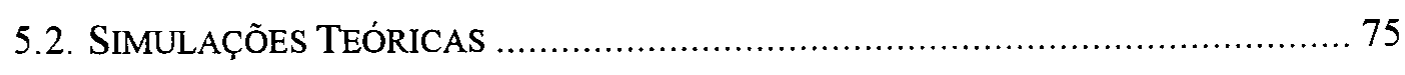

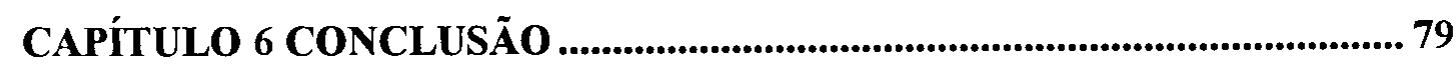

APÊNDICE A

A. 1. Tratamento Teórico da Técnica de Z-SCAN COM DOIS FEIXES ..............83 83

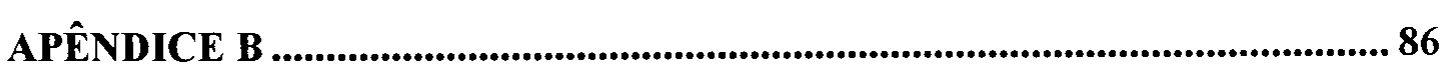

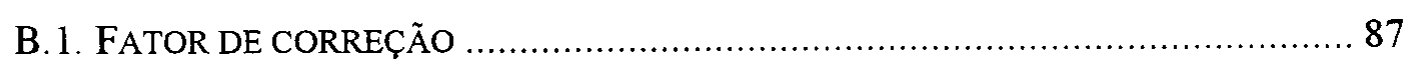

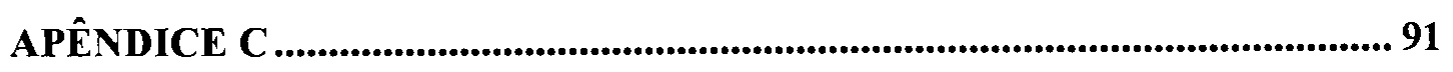

C. 1. CÁlCULO DA EXPRESSÃo QUE CONSIDERA O EFEITO DE LT E LP................. 92

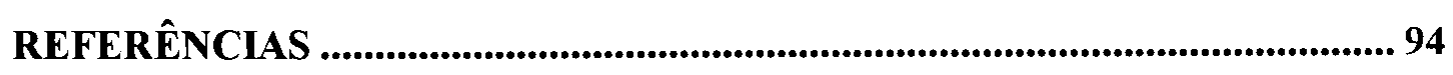

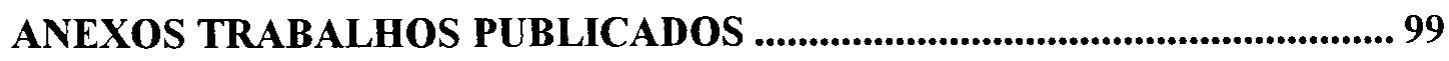




\section{LISTA DE FIGURAS}

Figura 2.1- Espectro de absorção do InSBZnGdN com $1 \%$ de $\mathrm{Nd}^{+3}$, espessura de (L

$=2.7 \mathrm{~mm}$ ) e $\mathrm{N}_{0}=1.97 \times 10^{20}$ ions $/ \mathrm{cm}^{3}$ 6

Figura 2.2- Diagrama de níveis de energia do $\mathrm{Nd}^{+3}[7]$ 8

Figura 2.3- Espectro de absorção e emissão do $\mathrm{SrAlF}_{5}: \mathrm{Cr}^{+3}$. A linha sólida representa a direção E ||$c$ e a linha pontilhada representa $E \| \mid a[8]$ 9

Figura 2.4- $\Delta \alpha$ em cristais dopados com $\mathrm{Cr}^{+3}$, versus a separação entre os níveis ${ }^{4} \mathrm{~T}_{2} \mathrm{e}$

${ }^{2} E[12]$

Figura 2.5- $\Delta \alpha$ do $\mathrm{Nd}^{+3}$ em diversos materiais[12]

Figura 2.6- Diagrama de Energia do Sistema de 4 Níveis

Figura 2.7- Diagrama simplificado dos níveis de energia do rubi [16] 14

Figura 3.1- Esquema experimental da técnica de varredura-z na qual a transmitância normalizada ao sinal de entrada é registrada como função da posição da amostra

Figura 3.2- Efeito de lente na técnica Z-scan para o caso em que $n_{2}>0$. Em (a) temos o caso em que a amostra está situada antes do foco $(z<0)$ e o feixe aparece expandido na posição da íris. Em (b) a amostra se encontra após o foco $(z>0)$ e o feixe tende a ser colimado. Em (c) temos a curva característica de Z-scan, onde $\mathrm{n}_{2}>0$. A linha pontilhada representa o feixe quando o efeito não linear é nulo ( $\mathrm{n}_{2}$ $=0$ ).

Figura 3.3- A Integral de Difração de Kirchhoff 24

Figura 3.4- Feixe gaussiano e técnica Z-scan $(d>>z)$ 26 
Figura 3.5- Curva característica da técnica de Z-scan $\operatorname{com} \mathrm{n}_{2}>0$ linha cheia e $\mathrm{n}_{2}<0$ linha pontilhada

Figura 3.6- Transmitância normalizada em função da posição da amostra, nos casos puramente refrativo e no caso puramente absorptivo. 36

Figura 3.7- Simulação teórica da varredura $Z$ para o caso de amostras que apresentam absorção de dois fótons. (a) medida com abertura da íris $\mathrm{S}<1$, (b) medida com a íris totalmente aberta $(\mathrm{S}=1)$ e $(\mathrm{c})$ razão entre as curvas a / c. 38

Figura 3.8- Distribuição de temperatura $\Delta \mathrm{T}(\mathrm{r})$ na Lente Térmica em função do tempo[20] 40

Figura 3.9- Arranjo dos feixes de excitação e prova na configuração do experimento de Lente Térmica com feixe duplo de modo descasado, onde $L_{1}$ e $L_{2}$ são as lentes e $w_{0 e}$ e $w_{0 p} 0$ raios dos feixes de excitação e prova respectivamente....... 42

Figura 4.1- Aparato experimental do experimento de LT. M1, M2, M3, M4 e M5 são os espelhos. 48

Figura 4.2- Posição da cintura do laser de He-Ne, $\lambda=632.8 \mathrm{~nm}$. 49

Figura 4.3- Posição da cintura do laser $\mathrm{Ar}, \lambda=514.5 \mathrm{~nm}$ 50

Figura 4.4- Desenvolvimento do sinal do feixe de prova na amostra $\mathrm{SrAlF}_{5}: \mathrm{Cr}^{+3} \mathrm{com}$ potência do laser $(P=600 \mathrm{~mW})$. Com o ajuste teórico obtivemos $\theta=-0.089$ e $t_{c}$ $=1.8 \mathrm{~ms}$.

Figura 4.5- Difusividade térmica para várias concentrações de $\mathrm{Nd}_{2} \mathrm{O}_{3}$ em vidros aluminato de cálcio com pouca sílica. 53

Figura 4.6- Aparato experimental utilizado para as medidas de varredura z. Onde as lentes 1,2 e 3 , tem distâncias focais $f=10 \mathrm{~cm}$ e as lentes 4 e 5 tem $f=4 \mathrm{~cm} . \ldots 54$ 
Figura 4.7- Evolução temporal do sinal de Z-scan da amostra de $\mathrm{GdAlO}_{3}: \mathrm{Cr}^{+3}\left(\mathrm{n}_{2}>\right.$ 0 ), colocada em (a) $z=0.85 z_{0}$, (b) $z=-0.85 z_{0}$ e (c) $z=7 z_{0}[10]$

Figura 4.8- Transmitância normalizada obtida na amostra $\mathrm{SrAlF}_{5}: \mathrm{Cr}^{+3}$ com potência do laser $(P=90 \mathrm{~mW})$, freqüência do chopper $(f=822 \mathrm{~Hz})$. Onde $S_{1}=100 \%$ abertura ( o ), $S_{2}=50 \%$ abertura $(\square)$ e razão $S_{2} / S_{1}(\bullet)$

Figura 4.9- Transmitância normalizada obtida na amostra $\mathrm{SrAlF}_{5}: \mathrm{Cr}^{+3}$ com potência do laser de $P=175 \mathrm{~mW}$ e freqüência do chopper $f=186 \mathrm{~Hz}$, onde $S_{1}=100 \%$ $(\mathrm{O})$ e $\mathrm{S}_{2}=50 \%(\bullet)$. O ajuste teórico com a expressão de LT forneceu $\mathrm{z}_{0}=2.6$ $\mathrm{mm}$. 59

Figura 4.10- Transmitância normalizada obtida no LiSAF com potência do laser de P $=95 \mathrm{~mW}$ ) e freqüência do chopper $\mathrm{f}=822 \mathrm{~Hz}$. A curva a) é o resultado da medida feita com $S_{1}=100 \%$ e a curva $b$ ) é o resultado da divisão da medida feita $\operatorname{com} S_{2}=50 \%$ por $S_{1}=100 \%$. 60

Figura 4.11- Medidas de Z-scan realizadas em várias potências do laser. O valor de $\Delta \Phi_{0}$ foi obtido através do ajuste de Z-scan realizado na curva $S_{2}=50 \%$ normalizado pela curva $S_{1}=100 \%$.

Figura 4.12- Medidas de absorção não-linear por Z-scan com abertura $S_{1}=100 \% \ldots 62$

Figura 4.13- Dependência do sinal com a amostra $\mathrm{SrAlF}_{5}: \mathrm{Cr}^{+3}$ na posição de vale $(\mathrm{z}=$ $-0.85 \mathrm{Z}_{0}$ ), com potência do laser $P=335 \mathrm{~mW}$ e freqüência do chopper $f=822$ Hz. A parte (a) mostra a medida com a íris aberta $\mathrm{S}_{1}=100 \%(\mathrm{O})$ e com a íris em $\mathrm{S}_{2}=50 \%(\square)$. A linha sólida representa um simples decaimento exponencial com $\tau=80 \mu$ s para $(\mathrm{O})$ 63

Figura 4.14- Razão das curvas $S_{2} / S_{1}(\bullet)$. O ajuste exponencial forneceu $\tau=67 \mu$ s. .. 64 
Figura 4.15- Medidas transiente no ponto de vale. Abertura da íris $S_{1}=100 \%$

Figura 4.16- Medidas de Z-scan resolvido no tempo do vidro fluorindato dopado com $\mathrm{Nd}^{+3}$, (a) com freqüência $(\mathrm{f}=840 \mathrm{~Hz})$ e potência $(\mathrm{P}=0.187 \mathrm{~W})$ e (b) com freqüência do chopper $(f=186 \mathrm{~Hz})$ e potência $(P=0.23 \mathrm{~W})$.

Figura 4.17- Medidas de Z-scan resolvido realizada com abertura $S_{1}=100 \%$, com freqüência $(f=840 \mathrm{~Hz})$ e potência $(P=0.187 \mathrm{~W})$. A linha cheia é o ajuste teórico.

Figura 4.18- Medida realizada no ZBLAN $1 \% \mathrm{Nd}^{+3}$. A curva com círculos fechados, curva (a), representa a medida com freqüência baixa $(f=186 \mathrm{~Hz})$, a curva $(b)$, círculo abertos, representa a medida com freqüência alta $(\mathrm{f}=824 \mathrm{~Hz})$. 68

Figura 4.19- Medida de Z-scan no YAG. Abertura da íris $\left(S_{2}=50 \%\right)$, potência do laser $(P=190 \mathrm{~mW})$ e freqüência do chopper $(f=824 \mathrm{~Hz})$. Características da amostra: $\mathrm{L}=1.4 \mathrm{~mm}, \tau_{0}=230 \mu \mathrm{s} \mathrm{e} \mathrm{I}_{\mathrm{s}}=78.3 \mathrm{KW} / \mathrm{cm}^{2}$

Figura 4.20- Medida de Z-scan no YAG. Abertura da íris $\left(S_{2}=50 \%\right)$, potência do laser $(P=190 \mathrm{~mW})$ e freqüência do chopper $(f=186 \mathrm{~Hz})$

Figura 5.1 - Simulações teóricas para o YAG, com $\Delta \Phi_{0}=0.06, \theta=0.013, \mathrm{t}_{\mathrm{c}}=12 \mu \mathrm{s}$ e $t=350 \mu$ s. A curva (a) representa a simulação onde é levado em conta os dois efeitos. A curva (b) contribuição do efeito de $L P(\theta=0)$ e a curva (c) o efeito de $\operatorname{LT}\left(\Delta \Phi_{0}=0\right)$ 77

Figura 5.2 - Simulações teórica. Em (a) temos a curva teórica de Z-scan e (b) a curva de Lente Térmica. .77

Figura 5.3 - Simulações do efeito de LT na Técnica Z-scan. .78 
Figura A.1- Simulações teórica para a expressão de Z-scan com diferentes valores do parâmetro $\mathrm{m}$. 85

Figura B.1 - A Figura (a) e (c) onde há a deformação na forma do sinal. A Figura (b) e (d) apresenta um caso ideal, sem deformação do sinal. .87

Figura B.2 - Forma do sinal com uma deformação devido ao tempo de abertura do chopper. 89

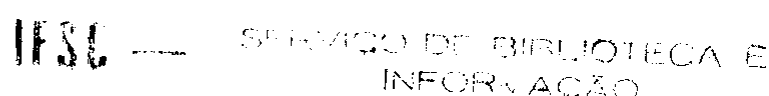
vii 


\section{LISTA DE TABELAS}

Tabela 4.1- Tabela com a composição das amostras utilizadas neste trabalho. 47

Tabela 4.2- Valores dos parâmetros obtidos.

Tabela 4.3- Valores de difusividade térmica obtidos pelo método de lente térmica no modo descasado 52

Tabela 5.1- Parâmetros usados para o cálculo da relação entre as diferenças de efeito de Lente de População e Lente Térmica, $\Delta \Phi_{0} / \Delta \phi_{\mathrm{th}}$ (Eq. 5.2) em diversos materiais. Os dados para o rubi foram retirados de [7]; para o YAG, YLF [25]; para o $\mathrm{LiCaAlF}_{6}: \mathrm{Cr}^{+3}$ de [12] e para o ZBLAN de[26]. 73

Tabela 5.2- Parâmetros usados para o cálculo da relação entre as diferenças de efeito de LP e LT, $\Delta \Phi_{0} / \Delta \phi_{\text {th }}$ (Eq. 5.2) em diversos materiais. $\mathrm{F}_{\mathrm{th}}=\mathrm{K}^{-1} \mathrm{ds} / \mathrm{dT}$ é um fator proporcional a $\Delta \phi_{\text {th }}$ e $F_{0}=\tau_{0} \Delta \alpha \mathrm{f}_{\mathrm{L}}{ }^{2} / \mathrm{n}$ é um fator proporcional a LP. Para estes cálculo foi utilizado $\mathrm{w}_{0}=20 \mu \mathrm{m}$. Os dados para o rubi foram retirados de [7]; para o YAG e YLF [25]; para o $\mathrm{LiCaAIF}_{6}: \mathrm{Cr}^{+3}$ de [12] e para o ZBLAN de [26].74 


\section{RESUMO}

Neste trabalho investigamos as duas contribuições mais importantes ao índice de refração não-linear, $\mathbf{n}_{2}$, em sólidos dopados: o efeito de Lente Térmica (LT) e o efeito de Lente de População (LP). Nós estudamos cristais fluoretos dopados com $\mathrm{Cr}^{+3}$ e vidros fluoretos dopados com $\mathrm{Nd}^{+3}$. As medidas de $\mathrm{n}_{2}$ foram feitas com a técnica de Z-scan resolvida no tempo, que é uma técnica simples mas muito sensível. Também realizamos medidas usando a técnica de Lente Térmica de modo descasado onde usou-se dois feixes de laser (um de excitação e outro de prova). Nós mostramos que pode-se separar temporalmente as contribuições a $\mathrm{n}_{2}$ devido a LT e LP, através da variação da freqüência do chopper. 


\begin{abstract}
In this work we investigated the two more important contributions to the index of no-lineal refraction, $\mathrm{n}_{2}$, in ion doped solid: the effects of Thermal Lens (LT) and Population Lens (LP). We studied $\mathrm{Cr}^{+3}$ doped fluoride crystals and $\mathrm{Nd}^{+3}$ doped fluoride glasses. The $n_{2}$ measurements were made with the time-resolved Z-scan technique, that is a simple but very sensitive technique. We also performed timeresolved Mode-mismatched Thermal Lens measurements, where two laser beams were used (one excitation beam and another probe beam). We showed that it is possible to temporally separate the contributions of LT and LP for $\mathbf{n}_{2}$, through the variation of the chopper frequency..
\end{abstract}




\section{CAPÍTULO 1}

INTRODUÇÃO 
$\mathrm{O}$ índice de refração não-linear, $\mathrm{n}_{2}$, de sólidos dopados têm sido estudadas por várias técnicas: medidas interferométricas, misturas de 4 ondas (degeneradas ou quase degeneradas), automodulação espacial de fase e biestabilidade ótica. Os sólidos dopados com $\mathrm{Nd}^{+3}$ e $\mathrm{Cr}^{+3}$ tem sido bastante estudados devido suas aplicações em lasers $[1,2]$. Nestes materiais as propriedades não-lineares ocorrem devido à população do estado excitado (metaestável) de íons dopantes, a qual tem uma susceptibilidade diferente da do estado fundamental. A parte real de $n_{2}, n_{2}$, é proporcional a diferença de polarizabilidade, $\Delta \alpha$, entre o estado excitado e o estado fundamental. A parte imaginária de $\mathrm{n}_{2}, \mathrm{n}_{2}$ ", é proporcional a diferença entre as seções de choque de absorção, $\Delta \sigma$, entre o estado excitado e o estado fundamental. Denominamos este processo de efeito de Lente de População (LP). Na maior parte dos sólidos dopados com $\mathrm{Cr}^{+3}$ ou $\mathrm{Nd}^{+3} \mathbf{n}_{2}$ ' é uma ordem de magnitude maior do que $\mathrm{n}_{2}$ ". Geralmente, parte da população do estado excitado decai via um processo nãoradiativo, de maneira que o laser aquece a amostra e portanto uma variação no caminho óptico é estabelecido devido ao coeficiente de temperatura do caminho óptico, ds/dT. Este efeito chamamos de efeito de Lente Térmica (LT)[3].

Neste trabalho apresentamos medidas do efeito de LT usando a técnica de Lente Térmica de modo descasado (feixe duplo[3]), uma técnica simples nãodestrutiva e muito sensível. Apresentamos também medidas do efeito de LP usando a técnica de Z-scan resolvida no tempo[4], que se mostrou muito superior a técnica de mistura de ondas. Obtivemos os primeiros resultados de $\Delta \alpha$ de $\mathrm{Nd}^{+3}$ em vidros fluoretos e cristais fluoretos dopados com $\mathrm{Cr}^{+3}$.

No caso de óxidos (cristais e vidros) geralmente a contribuição do efeito de LP é muito maior que a devido a LT. Entretanto, os materiais fluoretos (cristais e 
vidros) geralmente apresentam $\Delta \alpha$ e condutividade térmica muito menores que os óxidos, o que diminui o efeito de LP e aumenta a LT, respectivamente. Consequentemente, a LP e LT podem dar contribuições de mesma magnitude ao sinal de Z-scan. Nestes casos, é preciso discriminar estes dois efeitos para determinar $\Delta \alpha$. Mostraremos neste trabalho que os efeitos de LP e LT podem ser discriminados temporalmente (através da variação da freqüência do chopper)[5, 6].

No Capítulo 2 apresetamos uma breve discussão sobre as características espectroscópicas dos sólidos dopados com $\mathrm{Nd}^{+3}$ e $\mathrm{Cr}^{+3}$ e com uma revisão sobre a origem da não-linearidade em sólidos dopados. No Capítulo 3 apresentamos as técnicas de Z-scan (com o formalismo da integral de difração de Kirchhoff) e de Lente Térmica de modo descasado. No Capítulo 4 apresentamos os resultados experimentais obtidos com a técnica de Lente Térmica e Z-scan em diversos sólidos dopados e mostramos que é possível discriminar temporalmente a contribuição do efeito de LT do efeito de LP. No Capítulo 5 analisamos a contribuição do efeito térmico e eletrônico simultâneos apresentando um modelo teórico geral para o método resolvido no tempo (Z-scan) que inclui as duas contribuições LT e LP. O Capítulo 6 é a conclusão do trabalho. 


\section{CAPÍTULO 2}

\section{ESPECTROSCOPIA EM SÓLIDOS DOPADOS}




\subsection{Sintese dos vidros}

Durante o desenvolvimento deste trabalho realizamos medidas em vidros fluorindatos e viảros aluminato de cálcio dopados com $\mathrm{Nd}^{+3}$.

Os vidros fluorindatos foram preparados pelo Prof. Dr. R. Lebullenger no laboratório de vidros fluoretos do grupo de materiais do IFSC. O processo de preparação do vidro, após a pesagım dos vários componentes utilizados, consiste em fundir o material em cadinho de platina e verter o vidro líquido em um molde préaquecido. Em seguida, o conjunto "molde-vidro" sofre um recozimento a uma temperatura de 15 graus Celsius abaixo da temperatura de transição vítrea ( $\mathrm{Tg})$ da amostra afim de eliminar as tensões internas geradas durante a sua solidificação. Entretanto, no caso dos fluoretos existem muitas condições para a vitrificação ocorrer resultando um vidro de boa qualidade óptica: a água dever ser removida em cada estágio, os materiais de partida devem ser extremamente puros e a contaminação posterior proveniente de cadinhos ou inclusões externas devem ser prevenidas. Para evitar a contaminação por partículas do cadinho utiliza-se cadinho de platina ou de carbono vítreo. Ainda assim, pode ocorrer absorção de espécies químicas indesejáveis que serão liberadas durante a fusão. A presença de água e de outras impurezas aniônicas, tais como oxigênio, sulfatos e carbonatos podem influenciar drasticamente a formação vitrea ou provocar absorções indesejáveis no infravermelho. A produção de vidros fluoretos de qualidade óptica requer assim materiais de partida de alto grau de pureza, com fundição realizada em atmosfera seca e limpa. Na Fig. 2.1, representamos o espectro de absorção do vidro InSBZnGdN dopado com $1 \%$ de $\mathrm{Nd}^{+3}$. 


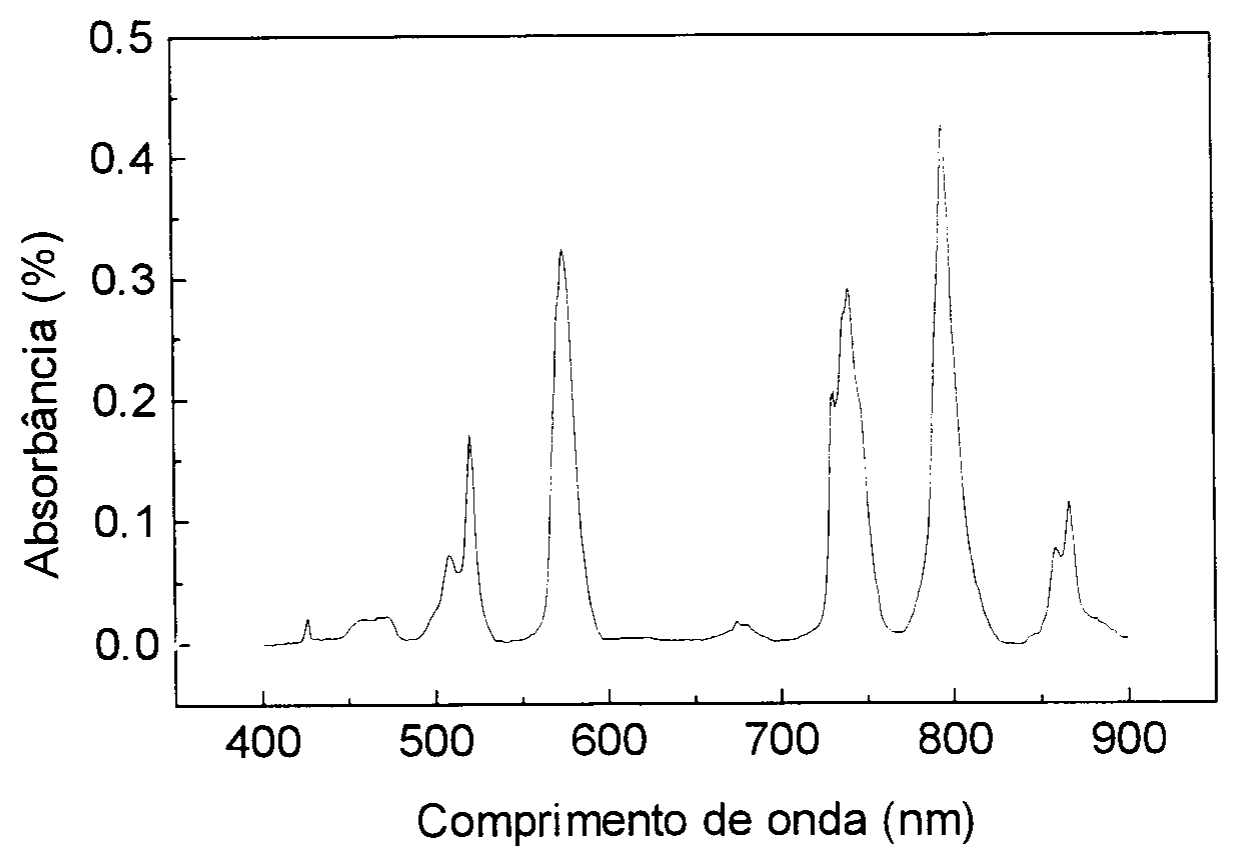

Figura 2.1- Espectro de absorção do InSBZnGdN com $1 \%$ de $\mathrm{Nd}^{+3}$, espessura de $(\mathrm{L}=2.7 \mathrm{~mm})$ e $\mathrm{N}_{0}=1.97 \times 10^{20}$ ions $/ \mathrm{cm}^{3}$.

Os vidros aluminato de cálcio foram preparados pelo estudante J. Sampaio, com a colaboração do Prof. M. Baesso e Prof. S. Gama nos laboratórios do IFGWUNICAMP. Estes vidros foram preparados à vácuo. Neste processo a diminuição da influência da água na estrutura do vidro obtido faz com que este material apresente maior transmissão na região do infravermelho. Este comportamento indica que este vidro é um candidato para aplicações em sistemas ópticos nesta região espectral, tais como janela óptica, filtro de absorção óptica e como meio ativo para lasers de estado sólido, este último, através da introdução de íons de terras raras em sua composição. No caso dos vidros dopados com terras raras é essencial que o vidro base apresente absorção óptica muito baixa na região de comprimentos de onda onde haverá a emissão do laser, obtendo-se uma melhor eficiência no processo de conversão de luz para luz reduzindo assim o efeito de lente térmica ou seja, a interferência dos efeitos térmicos durante a operação do laser. 


\subsection{Características Espectroscópicas de sólidos dopados com $\mathrm{Nd}^{+3}$}

A descoberta dos vidros fluoretos em 1975, desencadeou um grande interesse no estudo das propriedades óticas dos fluoretos de metais pesado dopados com terras raras. Já há alguns anos, observou-se que vidros baseados no $\mathrm{InF}_{3}$ são mais estáveis e apresentam uma maior janela de transparência na região do infravermelho $(\sim 8 \mu \mathrm{m})$.

Atualmente os lasers de estado sólido (não semicondutores) mais importantes são feitos com materiais dopados com $\mathrm{Nd}^{+3}$. Em relação ao rubi, eles apresentam a vantagem de funcionarem como laser de 4 níveis e por isso apresentam baixa condição de limiar ("threshold"). Além disso, apresentam alta seção de choque na transição laser por exemplo, o Nd ${ }^{+3}: \mathrm{YAG}\left(\mathrm{Y}_{3} \mathrm{Al}_{5} \mathrm{O}_{12}\right) \sigma=9 \times 10^{-19} \mathrm{~cm}^{2}(7$ vezes maior que a do rubi). Quando colocados numa estrutura vitrea o ín "sente" a variação do campo cristalino devido a estrutura amorfa do vidro. Por isso existe um grande alargamento inhomogêneo das linhas, ou seja, íons que "sentem" campos diferentes emitem em comprimentos de onda diferentes. No $\mathrm{Nd}^{+3}$ :YAG a largura de linha da transição laser é $6 \mathrm{~cm}^{-1}$ enquanto que num vidro este valor é da ordem de 50 vezes maior.

Entre os lasers de $\mathrm{Nd}^{+3}$, o YAG é o laser de estado-sólido mais utilizado. Ele possui uma combinação de propriedades unicamente favoráveis para a operação laser. O YAG, cristal hospedeiro é sólido, de boa qualidade ótica e possui uma alta condutividade térmica. Além disso, a sua estrutura cúbica favorece uma estreita largura de linha de fluorescência, a qual resulta em um alto ganho e um baixo limiar ("threshold") para operação laser. A estrutura[7] é estável desde baixas temperaturas até o ponto de fusão e transformações não tem sido verificadas na fase sólida. 
O YAG é um cristal opticamente isotrópico o qual possui uma estrutura cúbica característica de "garnetes". Em Nd:YAG cerca de $1 \%$ do $\mathrm{Y}^{+3}$ é substituído pelo $\mathrm{Nd}^{+3}$.

Na Fig. 2.2 temos a representação do diagrama dos níveis de energia para o $\mathrm{Nd}^{+3}$. Quando excitado em uma das bandas no visível o $\mathrm{Nd}^{+3}$ decai não-radiativamente para estado o metaestável ${ }^{4} \mathrm{~F}_{3 / 2}$ ( o nível laser superior) que tem um tempo de vida de $\sim 350 \mu \mathrm{s}$.

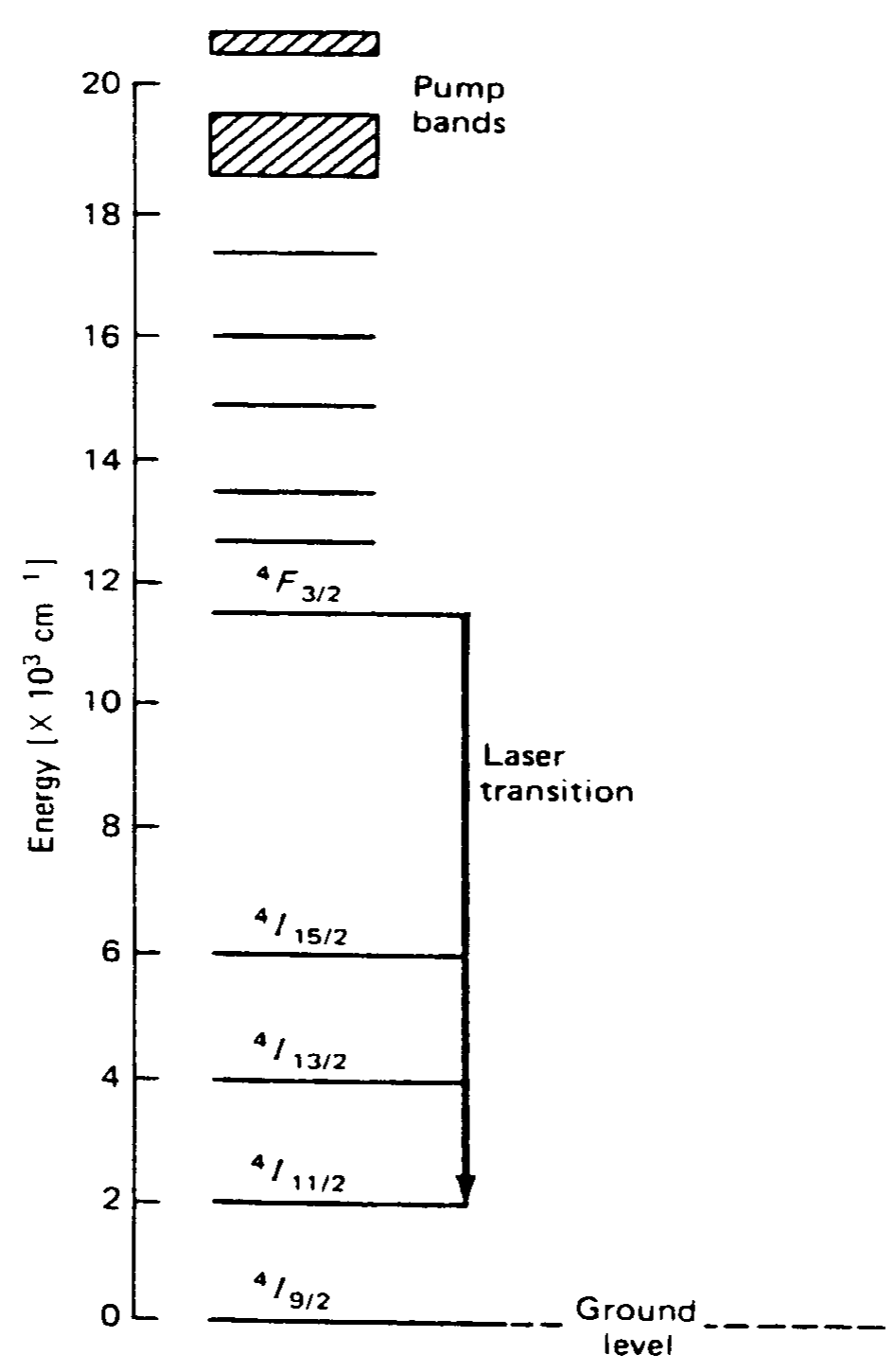

Figura 2.2- Diagrama de níveis de energia do $\mathrm{Nd}^{+3}$ [7] 


\subsection{Características Espectroscópicas de cristais dopados com $\mathrm{Cr}^{+3}$}

$\mathrm{O}$ íon $\mathrm{Cr}^{+3}$ possui a mesma estrutura eletrônica do $\mathrm{Ar}$ e mais três elétrons na camada de valência externa $3 \mathrm{~d}$. Os seus níveis de energia são bem conhecidos tanto no caso do íon livre como para o íon no campo cristalino.

No caso de cristais fluoretos dopados com $\mathrm{Cr}^{+3}$, existe um grande interesse em estudá-los devido ao fato de apresentarem bandas de absorção no vermelho, que podem ser excitadas por lasers de diodo em $670 \mathrm{~nm}$, e por apresentarem um largo intervalo de sintonia em torno de $800 \mathrm{~nm}$. Em geral, os fluoretos apresentam um campo cristalino fraco e por isso emitem em comprimentos de onda mais longos. $\mathrm{Na}$ Fig. 2.3 temos o espectro de absorção do $\mathrm{SrAlF}_{5}: \mathrm{Cr}^{+3}$. Este cristal apresenta um tempo de vida de $93 \mu$ s a $300 \mathrm{~K}$ e foi crescido pela equipe do Dr. H. Jenssen[8]

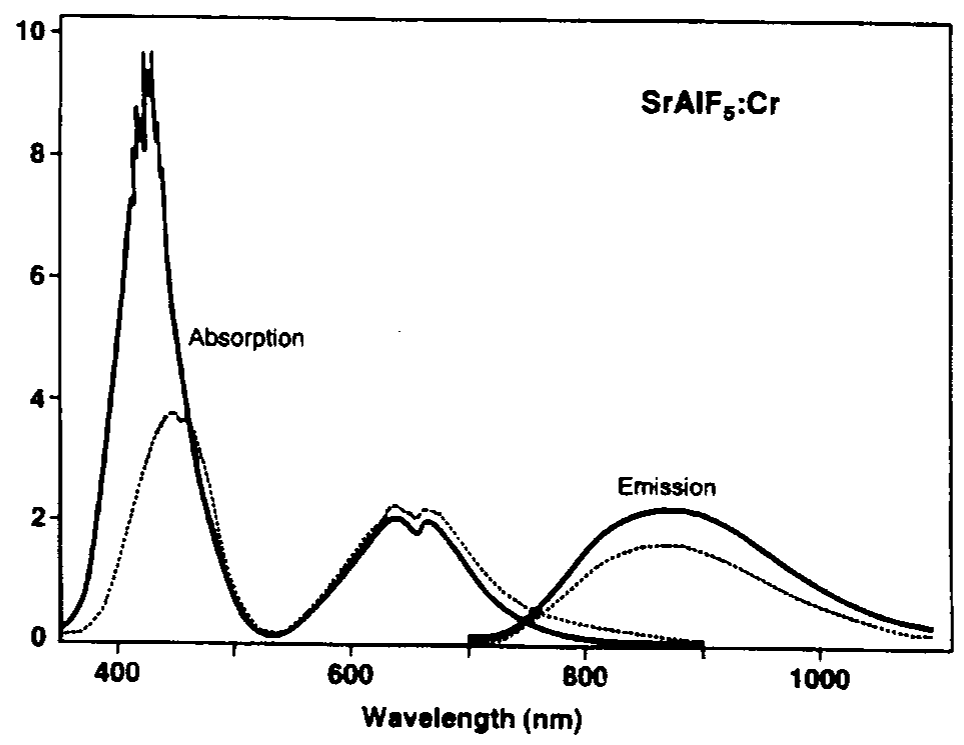

Figura 2.3- Espectro de absorção e emissão do $\mathrm{SrAlF}_{5}: \mathrm{Cr}^{+3}$. A linha sólida representa a direção $\mathrm{E} \| \mathrm{c}$ e a linha pontilhada representa $\mathrm{E} \| \mathrm{a}$ [8] 


\subsection{Investigação da origem da não-linearidade em Sólidos dopados}

Em sólidos dopados com $\mathrm{Nd}^{+3}$ e $\mathrm{Cr}^{+3}$, o efeito não-linear ocorre devido a diferença de polarizabilidade $\Delta \alpha$ entre o estado excitado e o estado fundamental. Veremos no capítulo 3, que fazendo uma medida de $\mathrm{n}_{2}$, , podemos encontrar o valor de $\Delta \alpha$. Apesar de ter sido bastante abordado por diversos grupos de pesquisas, a origem fisica de $\mathrm{n}_{2}$ 'a ainda continua sem uma total compreensão.

As propriedades não-lineares em sólidos dopados têm sido estudadas por várias técnicas. Como em outro materiais, a técnica Z-scan[4] tem se mostrado muito eficiente no estudo de sólidos dopados com íons $[1,9,10]$. Os sólidos dopados com íns $\mathrm{Cr}^{+3}$ e $\mathrm{Nd}^{+3}$ têm sido bastante estudados devido suas aplicações em lasers [1, 2] Nestes materiais, as propriedades não-lineares originam-se da população no estado metaestável do ín dopante, a qual apresenta uma susceptibilidade diferente do estado fundamental. A parte real do índice de refração não-linear é proporcional à diferença de polarizabilidade $\Delta \alpha$ entre o estado excitado e o estado fundamental. A parte imaginária do índice de refração é proporcional à diferença entre as sessões de choque de absorção do estado excitado e o estado fundamental. A este processo denominamos de efeito de Lente População (LP).

Geralmente, parte da população do estado excitado decai via um processo não-radiativo, de maneira que o laser aquece a amostra e portanto uma variação no caminho óptico é estabelecido devido ao coeficiente de temperatura do caminho óptico, ds/dT. A isto chamamos de efeito de Lente Térmica (LT)[3]. Os materiais fluoretos geralmente apresentam diferença de polarizabilidade $\Delta \alpha$ muito pequena e 
isto faz com que o efeito Lente População (LP) seja menor que o efeito de Lente Térmica (LT) $[2,11,12]$.

Os sólidos dopados com $\mathrm{Cr}^{+3}[9,10,12-15]$ e $\mathrm{Nd}^{+3}[2,11]$ foram estudados em diferentes matrizes. Nestes sólidos observou-se que $\Delta \alpha$ varia num intervalo de $10^{-26}-10^{-25} \mathrm{~cm}^{3}$ e sendo maior nos óxidos do que nos fluoretos (cristais e vidros). Os pesquisadores Weaver e Payne[12, 15] usaram a técnica de mistura de quatro ondas e mediram a diferença de polarizabilidade $\iota . \alpha$ em diferentes cristais dopados com $\mathrm{Cr}^{+3}$. Eles também estudaram cristais fluoretos, mas seus experimentos não apresentava sensibilidade o suficiente para a determinação dos valores obtidos de $\Delta \alpha$, eles obtiveram apenas um limite superior para $\Delta \alpha$. As Figs. (2.4) e (2.5) apresentam os resultados de $\Delta \alpha$ obtidos em cristais dopados com $\mathrm{Cr}^{+3}$ e vidros dopados com $\mathrm{Nd}^{+3}$, respectivamente.

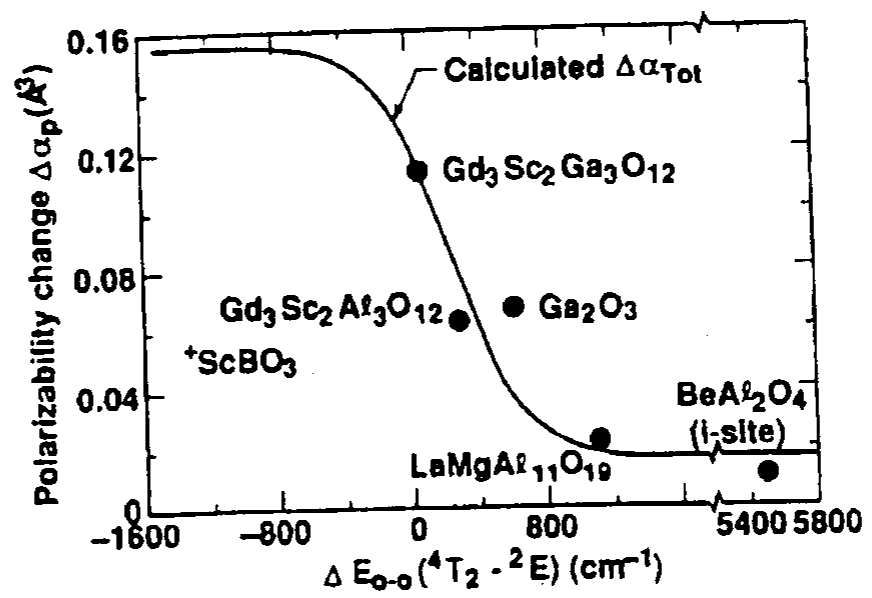

Figura 2.4- $\Delta \alpha$ em cristais dopados com $\mathrm{Cr}^{+3}$, versus a separação entre os niveis ${ }^{4} \mathrm{~T}_{2} \mathrm{e}{ }^{2} \mathrm{E}[12]$ 


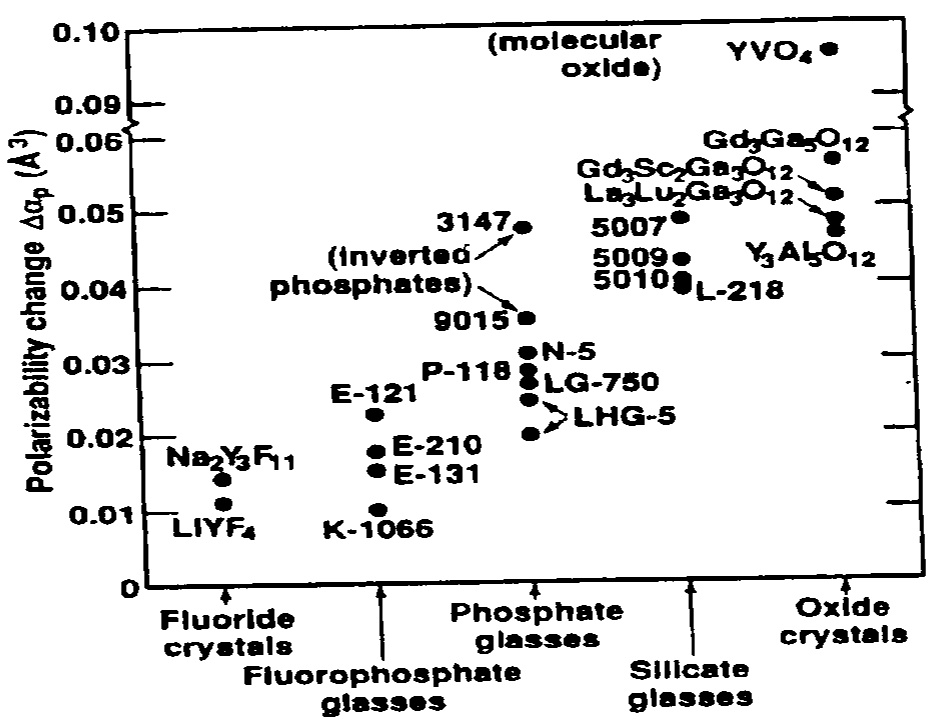

Figura 2.5- $\Delta \alpha$ do $\mathrm{Nd}^{+3}$ em diversos materiais[12]

\subsection{Equações de taxa}

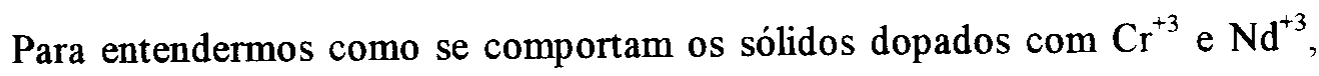
consideremos um sistema de 4 níveis de energia como mostra a Fig. 2.6, onde supomos que o sistema é excitado a partir do estado fundamental $G$ para um nível intermediário $\mathrm{I}_{1} \mathrm{o}$ qual decai rapidamente $\left(\sim \mathrm{fs}\right.$ ) para um estado metaestável $\mathrm{E}_{\mathrm{x}}$ (com tempo de vida $\tau_{0}$ da ordem de milisegundos). 


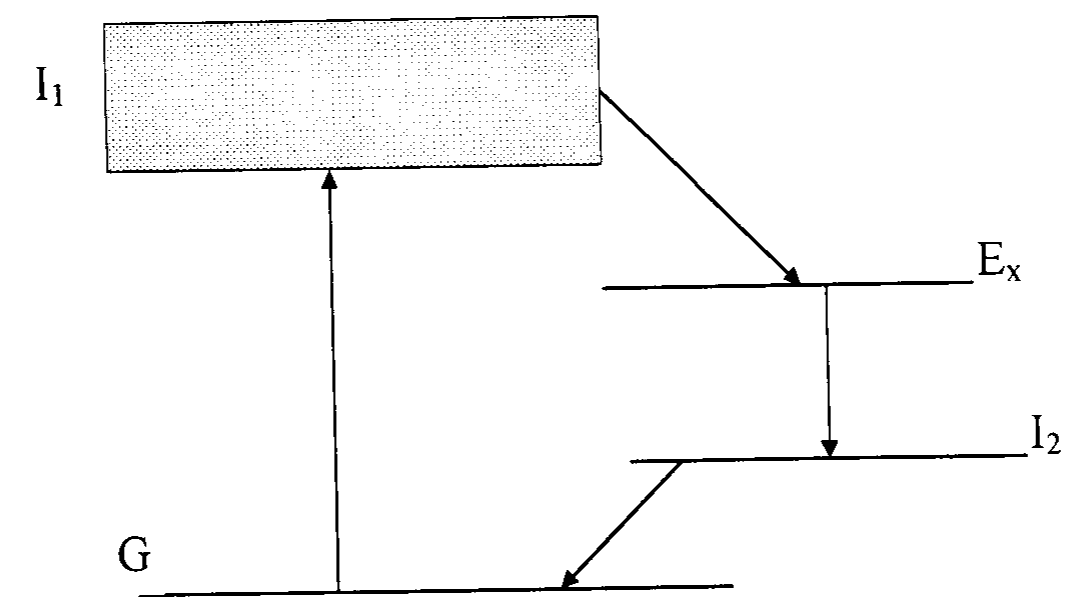

Figura 2.6- Diagrama de Energia do Sistema de 4 Níveis

No caso dos cristais dopados com $\mathrm{Cr}^{+3}$ o estado fundamental é o nivel ${ }^{4} \mathrm{~A}_{2}, \mathrm{o}$ estado intermediário $\mathrm{I}_{1}$ é a banda ${ }^{4} \mathrm{~T}_{2}$ e o estado metaestável $\mathrm{E}_{\mathrm{x}}$ é o nível ${ }^{2} \mathrm{E}$ (veja Fig.2.7). $O$ elétron é bombeado do nível metaestável $E_{x}$, como esta transição é muito rápida a população do nível $\mathrm{I}_{1}$ é desprezivel. No caso do $\mathrm{Cr}^{+3}$ o sistema pode se comportar como um sistema de 3 ou 4 níveis. Por exemplo o rubi se comporta como um sistema de 3 níveis onde no diagrama da Fig. 2.6 o estado $E_{x}$ decai diretamente para o estado fundamental $G$ (vide Fig. 2.7). Neste caso não existe o nível $I_{2}$. Já no caso dos fluoretos, $\mathrm{o}^{+3}$ se comporta como um sistema de 4 níveis.

Para os sólidos dopados com $\mathrm{Nd}^{+3}$ temos $\mathrm{E}_{\mathrm{x}}={ }^{4} \mathrm{~F}_{3 / 2}$, o qual pode decair diretamente para o estado fundamental $\mathrm{G}$ ou para outro estado intermediário $\left(\mathrm{I}_{2}\right)$ que corresponde aos niveis ${ }^{4} I_{15 / 2},{ }^{4} I_{13 / 2}$ e ${ }^{4} I_{11 / 2}$ (veja Fig. 2.2). Entretanto, uma vez que a diferença de energia $\Delta E=I_{2}-G$ é pequena $\left(\sim 2000 \mathrm{~cm}^{-1}\right)$, o sistema relaxa rapidamente para o estado fundamental com a emissão de fónons. 


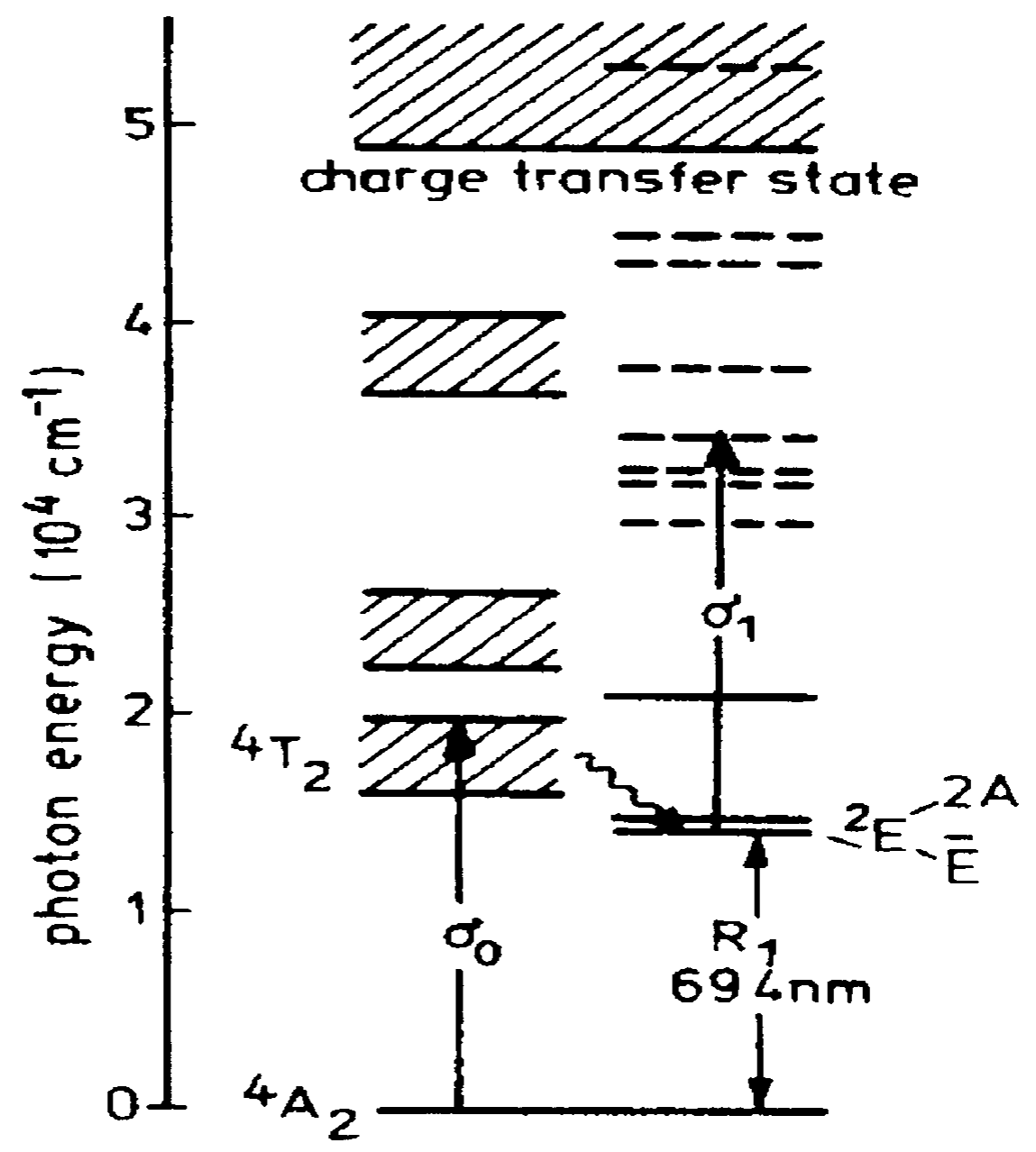

Figura 2.7- Diagrama simplificado dos níveis de energia do nubi [16]

Por meio das equações de taxa, podemos calcular as populações $\mathrm{N}_{\mathrm{G}}$ e $\mathrm{N}_{\mathrm{Ex}}$ correspondentes aos níveis $G$ e $E_{x}$. Uma vez que o tempo de vida dos níveis intermediários I é muito pequeno $(\sim \mathrm{ns}$, muito menor que o tempo de vida do estado Ex), podemos considerar $\mathrm{N}_{\mathrm{I} 1} \sim \mathrm{N}_{\mathrm{I} 2} \sim 0$. Portanto, trataremos apenas as populações dos estados excitado metaestável $\mathrm{N}_{\mathrm{Ex}}$ e fundamental $\mathrm{N}_{\mathrm{G}}$. Além disso desprezamos o decaimento direto do nível $I_{1}$ para o nível fundamental $G$, pois a taxa de transição do nível $\mathrm{I}_{1}$ para o nível $\mathrm{E}_{\mathrm{x}}$ é muito maior, desta forma podemos aproximar a 1 a eficiência quântica de bombeio do nível $\mathrm{E}_{\mathrm{x}}$. Desta maneira temos: 


$$
\begin{aligned}
\frac{\mathrm{dN}_{\mathrm{G}}}{\mathrm{dt}} & =-\frac{\sigma \mathrm{I}}{\hbar \omega} \mathrm{N}_{\mathrm{G}}+\frac{\mathrm{N}_{\mathrm{Ex}}}{\tau_{0}} \\
\frac{\mathrm{dN}_{\mathrm{Ex}}}{\mathrm{dt}} & =\frac{\sigma \mathrm{I}}{\hbar \omega} \mathrm{N}_{\mathrm{G}}-\frac{\mathrm{N}_{\mathrm{Ex}}}{\tau_{0}} \\
\mathrm{~N}_{0} & =\mathrm{N}_{\mathrm{G}}+\mathrm{N}_{\mathrm{Ex}}
\end{aligned}
$$

onde I é a intensidade de luz de freqüência $\omega$ na transição entre os níveis $G$ e $I_{1}, \sigma$ a seção de choque desta transição e $\tau_{0}$ o tempo de vida espontâneo no estado metaestável $E_{x}$. Como a população total $N_{0}$ é constante devemos ter

$$
\frac{\mathrm{dN}_{\mathrm{G}}}{\mathrm{dt}}=-\frac{\mathrm{dN}_{\mathrm{Ex}}}{\mathrm{dt}}
$$

como pode ser observado nas equações (2.1) e (2.3). Das Eqs. (2.3) e (2.2) obtemos:

$$
\frac{d N_{\mathrm{Ex}}}{\mathrm{dt}}=\frac{I}{\mathrm{I}_{\mathrm{s}}} \frac{\mathrm{N}_{0}}{\tau_{0}}-\frac{\left(1+\frac{\mathrm{I}}{\mathrm{I}_{\mathrm{s}}}\right)}{\tau_{0}} \mathrm{~N}_{\mathrm{Ex}}
$$

onde a intensidade de saturação $I_{s}$ é dada por:

$$
I_{s}=\hbar \omega / \sigma \tau_{0}
$$

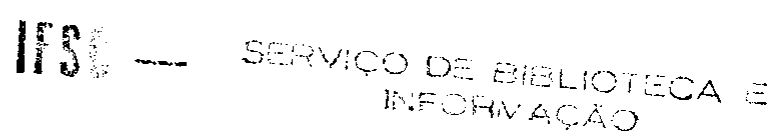


Supondo-se que em $\mathrm{t}=0$ o sistema está no estado fundamental, ou seja, $\mathrm{N}_{\mathrm{G}}=\mathrm{N}_{0}$ e $\mathrm{N}_{\mathrm{ex}}=0$, e neste instante é ligado o laser com intensidade I, das Eqs. (2.5) e (2.2) obtemos:

$$
\begin{aligned}
& N_{E x}(t)=N_{0} \frac{1 / I_{s}}{\left(1+I_{I}\right)}\left(1-e^{-t / \tau}\right) \\
& N_{G}(t)=N_{0} \frac{1 / I_{s}}{\left(1+\frac{I}{\left.I_{s}\right)}\left(1-e^{-t / \tau}\right)\right.}
\end{aligned}
$$

com

$$
\tau=\frac{\tau_{0}}{1+\mathrm{I} / \mathrm{I}_{\mathrm{s}}}
$$

Devido a distribuição da população entre o estado excitado $E_{x}$ e $o$ fundamental $\mathrm{G}$ a susceptibilidade do meio fica dada por:

$$
\chi=\frac{\mathrm{N}_{\mathrm{G}} \chi_{\mathrm{G}}+\mathrm{N}_{\mathrm{Ex}} \chi_{\mathrm{Ex}}}{\mathrm{N}_{0}}+\chi_{\mathrm{n}}
$$

que pela Eq. (2.3) podemos rescrever como:

$$
\chi \sim \chi_{\mathrm{m}}+\chi_{\mathrm{G}}+\left(\chi_{\mathrm{Ex}}-\chi_{\mathrm{G}}\right) \frac{\mathrm{N}_{\mathrm{Ex}}}{\mathrm{N}_{0}}
$$


onde $\chi_{\mathrm{j}}$ é a susceptibilidade do íon no estado $\mathrm{j}=\mathrm{G}$ ou $E_{\mathrm{x}}$. e $\chi_{\mathrm{m}}$ é a contribuição da matriz hospedeira (cristal ou vidro). O índice de refração complexo $\mathrm{n}$ pode ser calculado através da equação de Clausius-Mossotti (no sistema CGS):

$$
\frac{\mathrm{n}^{2}-1}{\mathrm{n}^{2}+2}=\frac{4 \pi}{3}\left(\frac{\mathrm{N}_{\mathrm{G}} \chi_{\mathrm{G}}+\mathrm{N}_{\mathrm{Ex}} \chi_{\mathrm{Ex}}}{\mathrm{N}_{0}}+\chi_{\mathrm{m}}\right)
$$

Sendo $\mathbf{n}_{0}$ o índice de refração linear, ou seja, o valor de $I=0\left(N_{G}=N_{0}\right.$ e $\left.N_{E x}=0\right)$, podemos escrever $\mathrm{n}=\mathrm{n}_{0}+\Delta \mathrm{n}$. Supondo $\Delta \mathrm{n}<<\mathrm{n}_{0}$, podemos expandir (2.10) e usando (2.6) e (2.7) obtemos:

$$
\begin{gathered}
\frac{\mathrm{n}_{0}^{2}-1}{\mathrm{n}_{0}^{2}+2}=\frac{4 \pi}{3}\left(\chi_{G}+\chi_{\mathrm{m}}\right) \\
\Delta \mathrm{n}=\frac{2 \pi}{9}\left(\frac{\mathrm{n}_{0}^{2}+2}{\mathrm{n}_{0}}\right)^{2}\left(\frac{\chi_{\mathrm{Ex}}-\chi_{\mathrm{G}}}{1+\mathrm{I}_{\mathrm{s}}}\right) \mathrm{I}(1-\exp [-t / \tau])
\end{gathered}
$$

No caso estacionário $(t>>\tau)$ podemos escrever:

$$
n=n_{0}+\frac{n_{2} I}{1+I_{s}}
$$

com

$$
\mathrm{n}_{2}=\frac{2 \pi}{9} \frac{\left(\mathrm{n}_{0}^{2}+2\right)^{2}}{\mathrm{n}_{0}} \frac{\left(\chi_{\mathrm{Ex}}-\chi_{\mathrm{G}}\right)}{\mathrm{I}_{\mathrm{s}}}
$$

e 


$$
\mathrm{f}_{\mathrm{L}}=\frac{n_{0}^{2}+2}{3}
$$

onde $\mathrm{f}_{\mathrm{L}}$ é conhecido como fator de Lorenz, que é responsável pela correção devido ao efeito do campo local no dielétrico.

A parte real de $\mathbf{n}_{2}=\mathbf{n}_{2}$ ' - in ${ }_{2}$ " é proporcional a diferença de polarizabilidade $\Delta \alpha$ entre os estados $E_{x}$ e G, de (2.14) e (2.15) obtemos:

$$
\mathrm{n}_{2}^{\prime}=\frac{2 \pi \mathrm{f}_{\mathrm{L}}^{2}}{\mathrm{n}_{0}} \mathrm{~N}_{0} \frac{\Delta \alpha}{\mathrm{I}_{\mathrm{s}}}
$$

A parte imaginária de $\mathbf{n}_{2}$ pode ser escrita em termo das seções de choque $\sigma_{E x} \mathrm{e}$ $\sigma_{\mathrm{G}}$ dos estados excitados e fundamental como:

$$
\mathrm{n}_{2}^{\prime \prime}=\frac{\lambda}{4 \pi} \mathrm{N}_{0} \frac{\Delta \sigma}{\mathrm{I}_{\mathrm{s}}}
$$

onde $\Delta \sigma=\sigma_{E x}-\sigma_{G}$. é a diferença entre as seções de choque de absorção dos dois estados. Portanto, a redistribuição das populações $N_{G}$ e $N_{E x}$ provoca uma alteração na parte real do índice de refração do meio.

As expressões acima são muito importantes neste trabalho, pois elas permitem a determinação de quantidades microscópicas como $\Delta \alpha$ e $\Delta \sigma$, através de medidas do parâmetro $\mathrm{n}_{2}$. Deve-se notar ainda a grande importância do fator de Lorenz $\mathrm{f}_{\mathrm{L}}$, principalmente nos cristais óxidos que apresentam um alto índice de refração $\left(\mathbf{n}_{0} \sim 2\right)$ e consequentemente um alto valor de $\mathrm{f}_{\mathrm{L}}$. 


\section{CAPÍTULO 3}

\section{TÉCNICAS DE Z-SCAN E LENTE TÉRMICA}




\subsection{Técnica de Z-scan}

Para a realização desta dissertação fizemos uso de duas importantes técnicas, técnica de Z-scan resolvida no tempo[17] e de Lente Térmica modo descasada[18]. A idéia básica de ambas as técnicas, consiste em relacionar a medida da variação da intensidade no centro do feixe no campo distante com a variação do índice de refração da amostra. Estas técnicas se destacam por sua simplicidade na montagem experimental e pela alta sensibilidade e por serem técnicas não contaminantes e não destrutivas.

A Fig.3.1 mostra o aparato experimental da técnica de varredura z. Um feixe laser que se propaga na direção $\mathrm{z}$ é focalizado no plano $\mathrm{z}=0$.

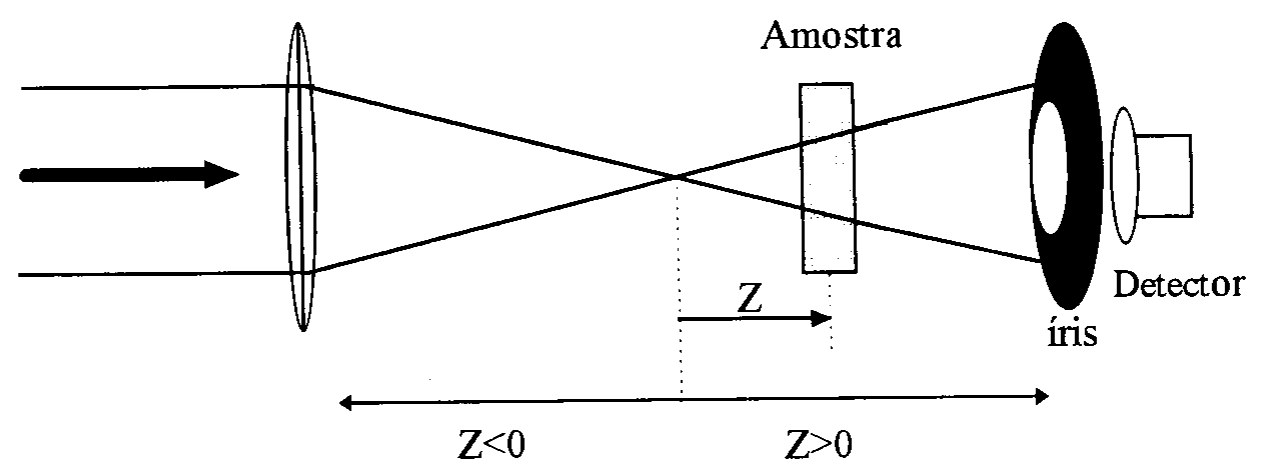

Figura 3.1- Esquema experimental da técnica de varredura-z na qual a transmitância normalizada ao sinal de entrada é registrada como função da posição da amostra $\mathbf{z}$.

A amostra é deslocada ao longo do eixo $\mathrm{z}$, varrendo a região focal do feixe. A amostra deve, em princípio, ser de espessura pequena comparada com o parâmetro 
confocal do feixe Gaussiano, $\mathrm{z}_{0}{ }^{1}$. Neste caso a lente induzida pode ser considerada, em primeira aproximação, como uma lente fina, cuja distância focal equivalente depende da posição da amostra em relação ao plano focal e cujo efeito é o de colimar ou focalizar ainda mais o feixe incidente, dependendo da posição da amostra, fazendo com que haja uma diminuição ou aumento na intensidade da luz que passar pela íris da Fig.3.1.

A variável que se mede em ıma varredura $\mathrm{z}$, é a potência transmitida através da íris que é posicionada em frente ao detector como função da posição $\mathrm{z}$ da amostra. O resultado de uma medida se expressa em termos da Transmitância Normalizada, $\mathrm{T}(\mathrm{z})$, definida como a razão entre $\mathrm{P}(\mathrm{z})$ e a potência transmitida com a amostra longe do foco, onde a intensidade é suficientemente pequena para que possamos desprezar qualquer efeito não linear:

$$
T(z)=\frac{P(z)}{P\left(|z|>>z_{0}\right)}
$$

A Fig. 3.2 permite o entendimento qualitativo do resultado de uma varredura$\mathrm{z}$ para o caso em que a amostra possui $\mathrm{n}_{2}>0$, onde a lente induzida é convergente. Começando a varredura longe do foco $\left(\mathrm{z}<<-\mathrm{z}_{0}\right)$ onde a intensidade do laser é fraca para que se possa observar efeitos não-lineares, a intensidade transmitida permanece relativamente constante $T(z)=1$. Ao aproximar a amostra do plano focal do feixe incidente (linha pontilhada vertical Fig. 3.2), o efeito de auto-focalização aumenta o índice de refração no centro do feixe, que chamamos de efeito de lente induzida na

\footnotetext{
${ }^{1} \mathrm{O}$ parâmetro $\mathrm{z}_{0}$ também é conhecido como intervalo de Rayleigh.
} 
amostra. A amostra se comporta como uma lente convergente, que colocada antes do plano focal $(z<0)$ faz com que o feixe incidente focalize antes do plano $z=0$, tornando-se mais expandido na posição da íris. Desta forma, a transmitância medida tende a diminuir. Por outro lado, uma lente convergente colocada na frente do foco $(z>0)$ colima o feixe aumentando a transmitância medida. Finalmente em $z>>z_{0} a$ transmitância novamente volta a tender ao valor 1. Esta análise mostra que a transmitância em função de $z$ possui um mínimo em $z<0$ e um máximo em $z>0$.
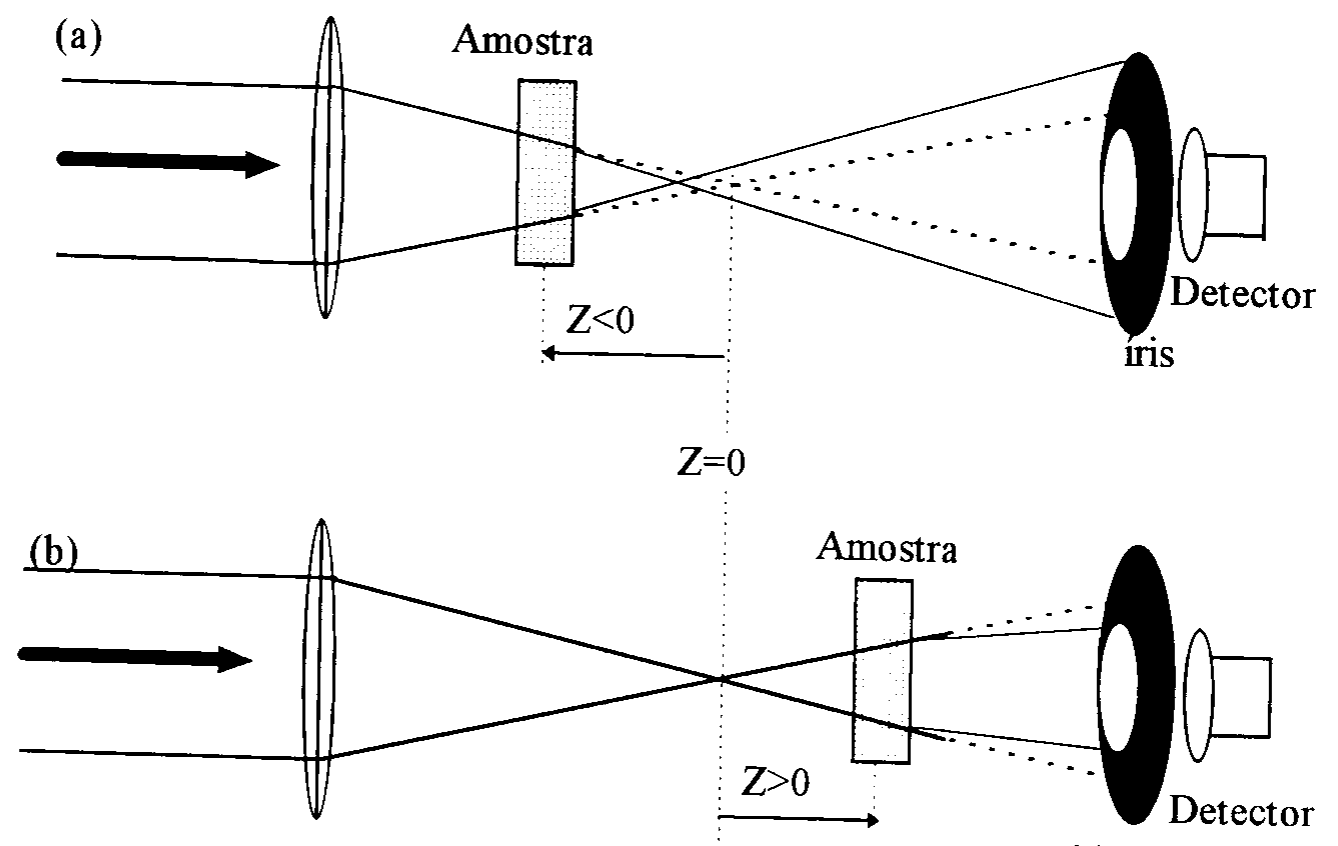

(c)

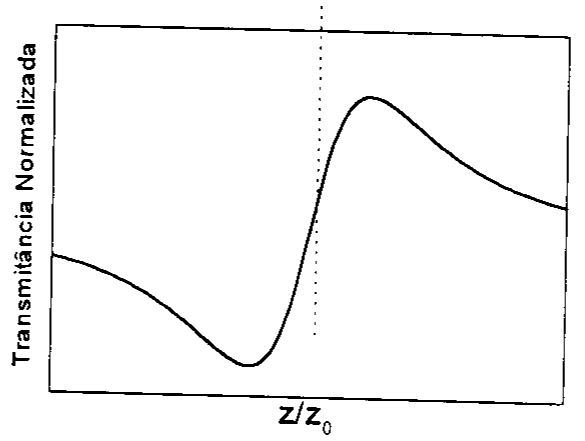

iris

Figura 3.2- Efeito de lente na técnica Z-scan para o caso em que $n_{2}>0$. Em (a) temos o caso em que a amostra está situada antes do foco $(z<0)$ e o feixe aparece expandido na posição da íris. Em (b) a amostra se encontra após o foco $(z>0)$ e o feixe tende a ser colimado. Em (c) temos a curva característica de $Z$-scan, onde $\mathrm{n}_{2}>0$. A linha pontilhada representa o feixe quando o efeito não linear é nulo $\left(\mathrm{n}_{2}=0\right)$ 
De maneira análoga, para o caso em que o meio apresenta $n_{2}<0$, podemos verificar que o comportamento será o oposto ao caso $n_{2}>0$, ou seja, o meio se comporta como uma lente divergente e as posições do máximo e do mínimo de transmitância estarão invertidâs em relação ao caso anterior $\left(\mathbf{n}_{2}>0\right)$.

É importante salientar que a técnica de Z-scan é um método simples de se medir uma mudança de fase do campo elétrico da luz. No campo afastado do feixe, a transmitância através da íris é uma medida direta da redistribuição de intensidade induzida pela não linearidade. Esta redistribuição é devida à mudança de fase induzida no campo próximo, isto é, na amostra que se encontra próxima do foco. Isto faz com que a técnica de Z-scan tenha limitações em relação a outras técnicas onde a amostra permanece fixa.

Como toda amostra exibe irregularidades nas suas superficies e inomogeneidades no seu volume, a fase das frentes de onda sofrem mudanças de forma independente da intensidade, a medida que a amostra se desloca ao longo do eixo z. Se estas mudanças forem pequenas é possível subtrair os seus efeitos das medidas. Porém, se forem grandes, podem alterar o perfil gaussiano da intensidade no interior da amostra e a interpretação dos resultados se torna inviável.

\subsection{A Integral de Difração de Kirchhoff}

A integral de difração de Kirchhoff é usada em ótica linear para o cálculo de figuras de difração e outras aplicações. Iremos mostrar que o cálculo da propagação de um feixe gaussiano pode ser feito, de maneira aproximada, para o campo distante usando a integral de difração de Kirchhoff. Além disso, ela pode ser usada para 
descrever os fenômenos de Modulação Transversal de Fase (que é usado no tratamento da técnica de Z-scan) e Lente Térmica.

Consideremos a difração de uma onda plana monocromática, que se propaga na direção dada pelo versor $\mathbf{n}$, e é difratada por um orificio finito $E$ no plano $A$, num plano opaco infinito B (Fig. 3.3). Supomos que o orificio seja plano, sejam ( $\left.\mathrm{x}_{0}, \mathrm{y}_{0}\right)$ as coordenadas do orificio no plano $A$ e $\left(x_{1}, y_{1}\right)$ as coordenadas no plano B de observação, a uma distância d do crificio. A amplitude do campo $\varepsilon_{B}$ num ponto $P_{1}$ do plano B de observação é dada pela soma dos campos $\varepsilon_{\mathrm{A}}$ devido a todos os pontos $\mathrm{P}_{0}$ do plano A do orificio.

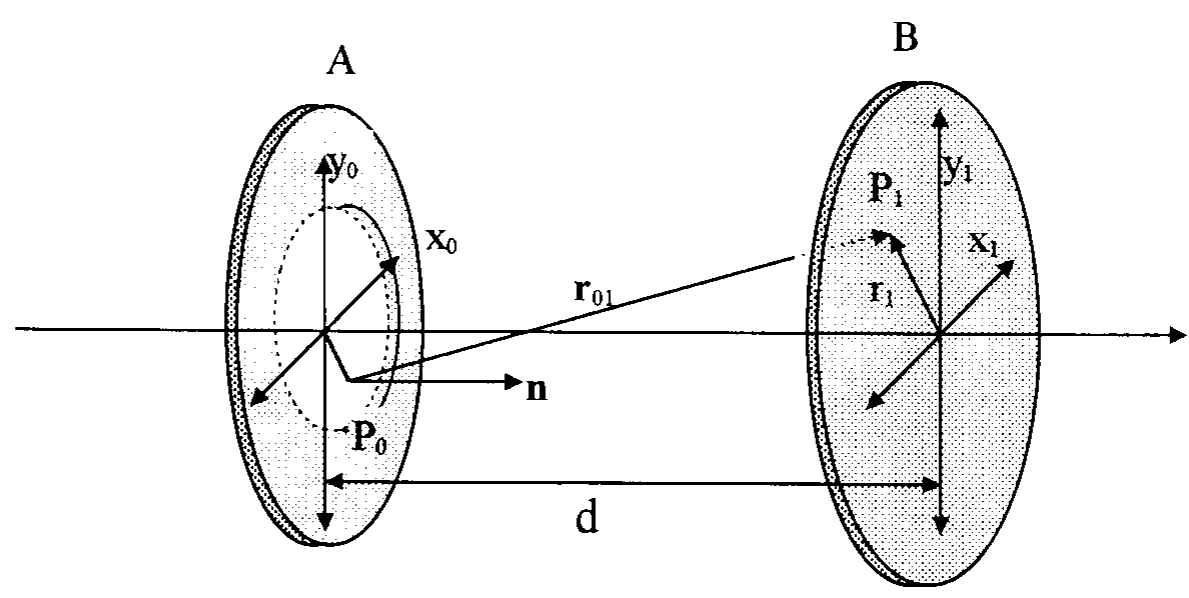

Figura 3.3- A Integral de Difração de Kirchhoff

Matematicamente esta idéia é expressa pela integral de Kirchhoff dada por:

$$
\varepsilon_{\mathrm{B}}\left(\mathrm{x}_{1}, \mathrm{y}_{1}\right)=\frac{\mathrm{i}}{\lambda} \iint_{\mathrm{E}} \frac{\exp \left[-\mathrm{ikr}_{01}\right]}{\mathrm{r}_{01}} \cos \left(n \cdot \mathrm{r}_{01}\right) \varepsilon_{\mathrm{A}}\left(\mathrm{x}_{0}, \mathrm{y}_{0}\right) \mathrm{dx}_{0} \mathrm{dy} \mathrm{y}_{0}
$$

onde $\lambda$ é o comprimento de onda da luz. Para resolver a integral acima faz-se várias aproximações que são válidas se a distância d for muito maior que a máxima 
dimensão linear da abertura $E$ ou seja $r_{0}<<d\left(r_{0}^{2}=x_{0}^{2}+y_{0}^{2}\right)$ implicando que as aproximações são válidas para pequenos ângulos do vetor $\mathbf{r}_{01}$. Nosso interesse é estudar problemas com simetria cilíndrica onde a amplitude do campo incidente é dada por $\varepsilon_{\mathrm{A}}\left(\mathrm{x}_{0}, \mathrm{y}_{0}\right)=\varepsilon_{\mathrm{A}}\left(\mathrm{r}_{0}\right)$ e a do campo no plano $\mathrm{B}$ de observação por $\varepsilon_{\mathrm{B}}\left(\mathrm{x}_{1}, \mathrm{y}_{1}\right)=$ $\varepsilon_{\mathrm{B}}\left(\mathrm{r}_{1}\right), \operatorname{com} \mathrm{r}_{1}^{2}=\mathrm{x}_{1}^{2}+\mathrm{y}_{1}^{2}$. Neste caso pode-se mostrar que a aproximação de Fresnel a expressão (3.2) fica:

$$
\varepsilon_{\mathrm{B}}\left(\mathrm{r}_{1}\right)=\frac{\mathrm{ik}}{\mathrm{d}} \int_{0}^{\infty} \exp \left[-\mathrm{ikr}_{0}^{2} / 2 \mathrm{~d}\right] \varepsilon_{\mathrm{A}}\left(\mathrm{r}_{0}\right) \mathrm{J}_{0}\left(\frac{\mathrm{kr}_{0} \mathrm{r}_{1}}{\mathrm{~d}}\right) \mathrm{r}_{0} \mathrm{dr}_{0}
$$

onde $k=2 \pi / \lambda$ é o módulo do vetor de onda

Consideremos agora o caso em que o campo incidente é um feixe gaussiano $\mathrm{TEM}_{\mathrm{oo}}$. Por conveniência, escrevemos a distância $\mathrm{z}$, entre a cintura do feixe e o plano $A$, em termos de uma nova variável adimensional $x=z / z_{0}$, onde $z_{0}=\pi w_{0}{ }^{2} / \lambda$ é a chamada distância confocal do feixe. A amplitude do campo fica dada por:

$$
\varepsilon_{\mathrm{A}}\left(r_{0}, x\right)=\varepsilon_{0} \frac{w_{0}}{w(\mathrm{x})} \exp \left[-\frac{r_{0}^{2}}{w^{2}(\mathrm{x})}-i \frac{k r_{0}^{2}}{2 R(\mathrm{x})}+i \tan ^{-1}(\mathrm{x})\right]
$$

onde w é chamado "waist", R o raio de curvatura. Os valores de w e R dependem de $\mathrm{z}$ de acordo com:

$$
\begin{aligned}
& w^{2}(x)=w_{0}^{2}\left(1+x^{2}\right) \\
& R(x)=\left(z_{0} / x\right)\left(1+x^{2}\right)
\end{aligned}
$$


onde $\mathrm{w}_{0}$ é o raio do feixe no ponto de máxima intensidade $(\mathrm{z}=0)$. Da mesma forma para a distância ao plano de observação B podemos definir a variável adimensional $x_{B}=\left(z^{+}+d\right) / z_{0}$ e teremos que $R(z)$ e $w(z)$ no plano $B$ são dados por:

$$
\begin{aligned}
& \mathrm{w}^{2}\left(\mathrm{x}_{\mathrm{B}}\right)=\mathrm{w}_{0}{ }^{2}\left(1+\mathrm{x}_{\mathrm{B}}{ }^{2}\right) \\
& \mathrm{R}\left(\mathrm{x}_{\mathrm{B}}\right)=\left(\mathrm{z}_{0} / \mathrm{x}_{\mathrm{B}}\right)\left(1+\mathrm{x}_{\mathrm{B}}{ }^{2}\right)
\end{aligned}
$$

Supomos que este feixe gaussiano incide numa amostra a uma distância $z$ da origem (o ponto $\mathrm{z}=0$ onde a intensidade axial do feixe é máxima) tal como mostra a Fig. 3.4. Supomos ainda que a amostra é opticamente fina de tal forma que, sendo $\varepsilon_{\mathrm{A}}(\mathrm{r})$ o campo incidente, na saída da amostra o campo é dado por:

$$
-\varepsilon_{A}^{\prime}\left(r_{0}\right)=\varepsilon_{A}\left(r_{0}\right) \exp [-i \Delta \phi]
$$

onde $\Delta \phi=\Delta \phi\left(\mathrm{r}_{0}\right)=\Delta \mathrm{n}\left(\mathrm{r}_{0}\right) \mathrm{kL}$ é uma variação de fase provocada pelo perfil de índice de refração $\Delta \mathrm{n}\left(\mathrm{r}_{0}\right)$ induzido pelo laser.

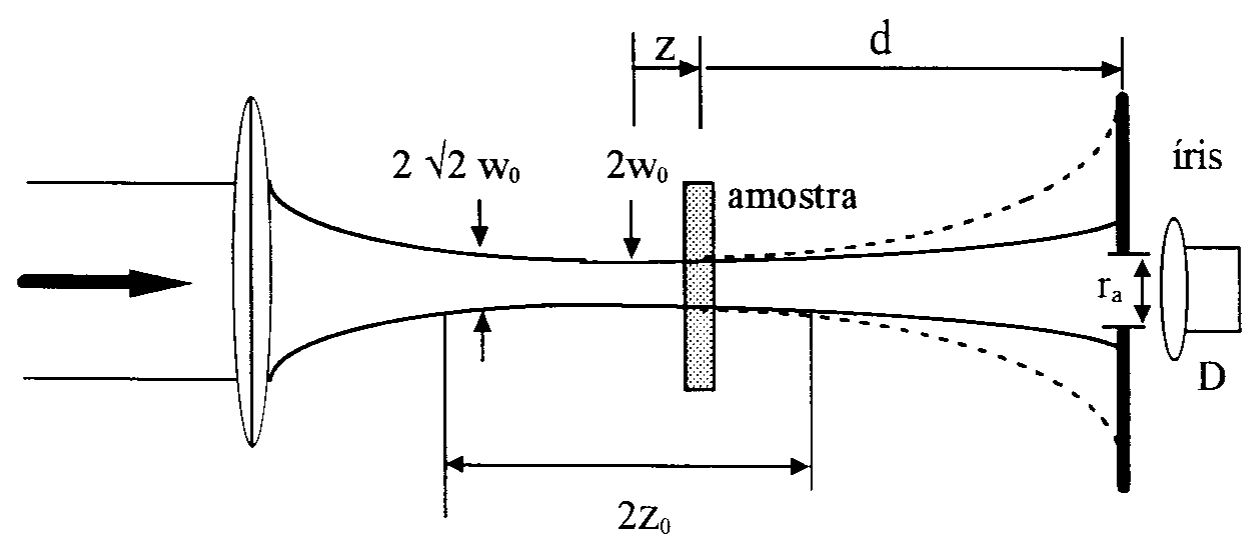

Figura 3.4- Feixe gaussiano e técnica Z-scan $(\mathrm{d}>>\mathrm{z})$ 
O cálculo que faremos agora é válido para qualquer perfil de índice de refração com simetria radial $\Delta \mathbf{n}(\mathrm{r})$, ou num caso mais geral qualquer perfil de fase $\Delta \phi\left(\mathrm{r}_{0}\right)$ (por exemplo devido ao efeito de LT ou LP). Colocando $\varepsilon_{\mathrm{A}}{ }^{\prime}\left(\mathrm{r}_{0}\right)$ no lugar de $\varepsilon_{\mathrm{A}}\left(\mathrm{r}_{0}\right)$ na Eq.(3.3) e considerando que o diâmetro do feixe não muda ao passar pela amostra, teremos:

$$
\varepsilon_{\mathrm{B}}\left(\mathrm{r}_{1}\right)=\frac{\mathrm{ik} \varepsilon_{0}}{\mathrm{~d}} \int_{0}^{\infty} \exp \left[-\mathrm{i}\left(\Phi_{\mathrm{D}}+\Delta \phi\left(\mathrm{r}_{0}\right)\right)\right] \exp \left[-r_{0}^{2} / w_{0}^{2}\right] \mathrm{J}_{0}\left(\frac{\mathrm{kr}_{0} \mathrm{r}_{1}}{\mathrm{~d}}\right) \mathrm{r}_{0} \mathrm{dr} \mathrm{r}_{0}
$$

onde

$$
\begin{gathered}
\Phi_{\mathrm{D}}=\mathrm{kr}_{0}^{2}\left(\frac{1}{2 \mathrm{~d}}+\frac{1}{2 \mathrm{R}_{\mathrm{A}}}\right) \\
\Delta \phi\left(\mathrm{r}_{0}\right)=\Delta \mathrm{n}\left(\mathrm{r}_{0}\right) \mathrm{kL}
\end{gathered}
$$

Veremos em seguida que a integral da Eq. (3.9) apresenta solução analítica para o caso em que $\Delta \phi\left(\mathrm{r}_{0}\right)=0$. Entretanto no caso onde $\Delta \phi\left(\mathrm{r}_{0}\right)=\mathrm{kL} \Delta \mathrm{n}\left(\mathrm{r}_{0}\right)=$ $\mathrm{kLn}_{2} \mathrm{I}_{0} \exp \left(-2 \mathrm{r}_{0}{ }^{2} / \mathrm{w}^{2}\right)$, a integral precisa ser feita numericamente. No problema do Z-scan em geral considera-se que o efeito não-linear é pequeno $\left(\Delta \phi\left(\mathrm{r}_{0}\right)<<\pi\right)$ de tal forma que podemos fazer a seguinte aproximação na Eq. (3.8):

$$
\exp \left[-\mathrm{i} \Delta \phi\left(\mathrm{r}_{0}\right)\right] \sim 1-\mathrm{i} \Delta \phi\left(\mathrm{r}_{0}\right)
$$

esta aproximação simplificará significativamente os cálculos e desta maneira podemos obter resultados analíticos. Consideremos agora o caso $\mathrm{kr}_{0}{ }^{2} / 2 \mathrm{~d} \sim 0$, o que equivale a difração de Fraunhofer. Neste caso substituindo (3.6) em (3.10) temos 
$\Phi_{\mathrm{D}}=\mathrm{r}_{0}{ }^{2} \mathrm{x} / \mathrm{w}^{2}(\mathrm{x})$. Usando as Eqs. (3.4), (3.5) e (3.6) e escrevendo $\Phi_{\mathrm{D}}$ em termos de uma nova variável $y=r_{0} / w(x)$, a Eq. (3.9) pode ser rescrita como:

$$
\varepsilon_{B}\left(r_{1}\right)=\frac{i k \varepsilon_{0} w^{2}(x)}{d} \int_{0}^{\infty} \exp \left[-i y^{2}(-i+x)\right][1-i \Delta \phi(y)] J_{0}(\beta y) y d y
$$

onde $\beta=\mathrm{kr}_{1} \mathrm{w}(\mathrm{x}) / \mathrm{d}$

A integral (3.13) pode ser separada em duas integrais:

$$
\varepsilon_{B}\left(r_{1}\right)=\frac{i k \varepsilon_{0} w^{2}(x)}{d}[H+\delta H]
$$

onde

$$
\begin{gathered}
H=\int_{0}^{\infty} \exp \left[-y^{2}(1+i x)\right]_{0}(\beta y) y d y \\
\delta H=-i \int_{0}^{\infty} \exp \left[-y^{2}(1+i x)\right] \Delta \phi(y) J_{0}(\beta y) y d y
\end{gathered}
$$

Resolvendo para o caso geral em que $\Delta \phi\left(r_{0}\right) \neq 0$, chegaremos a forma do perfil de intensidade no campo distante $\mathrm{I}_{\mathrm{B}} \propto\left|\varepsilon_{\mathrm{B}}\left(\mathrm{r}_{1}\right)\right|^{2}$ dada por:

$$
\left|\varepsilon_{\mathrm{B}}\left(\mathrm{r}_{1}\right)\right|^{2} \propto|\mathrm{H}+\delta \mathrm{H}|^{2} \sim|\mathrm{H}|^{2}+\mathrm{H} \delta \mathrm{H}^{*}+\mathrm{H}^{*} \delta \mathrm{H}
$$

onde a aproximação acima é feita considerando que $\Delta \phi\left(\mathrm{r}_{0}\right)$ é pequeno, logo $\delta \mathrm{H}<<\mathrm{H}$ e podemos desprezar o termo $|\delta \mathrm{H}|^{2}$. Além disso, experimentalmente é conveniente 
trabalhar com a transmitância normalizada ao caso $\Delta \phi\left(\mathrm{r}_{0}\right)=0$, que é dada por $\mathrm{T}=|\mathrm{H}+\delta \mathrm{H}|^{2} /|\mathbf{H}|^{2}$, a qual pode ser expressa como:

$$
\mathrm{T}=1+2 \operatorname{Re}\{\delta \mathrm{H} / \mathrm{H}\}
$$

onde $\operatorname{Re}\{\}$ significa a parte real do argumento.

\subsection{Tratamento Teórico da Técnica Z-scan}

Consideremos um meio não-linear onde o índice de refração é dado por $\mathbf{n}=\mathbf{n}_{0}$ $+\mathrm{n}_{2} \mathrm{I}$, onde $\mathrm{I}=\mathrm{I}\left(\mathrm{r}_{0}, \mathrm{z}\right)$ é o perfil de intensidade radial do feixe e $\mathrm{z}$ é a distância da amostra ao ponto de máxima intensidade axial. O perfil de intensidade gaussiano induz no meio não-linear um perfil de índice de refração também gaussiano $\Delta \mathbf{n}\left(\mathrm{r}_{0}, \mathrm{z}\right)=$ $\mathbf{n}_{2} \mathbf{I}\left(\mathrm{r}_{0}, \mathrm{z}\right) \propto\left|\varepsilon\left(\mathrm{r}_{0}, \mathrm{z}\right)\right|^{2}$ o qual é dado pelas Eqs. (3.4), (3.5) e (3.6). Tal como na seção (3.2), escreveremos as coordenadas $\mathrm{z}$ e $\mathrm{r}$ em termos das variáveis adimensionais $x=z / z_{0}$ e $y=r_{0} / w$. Logo o perfil de fase $\Delta \phi(y, x)$ fica dado por:

$$
\Delta \phi(\mathrm{y}, \mathrm{x})=\Delta \phi_{0}(\mathrm{x}) \exp \left[-2 \mathrm{y}^{2}\right]
$$

com

$$
\Delta \phi_{0}(\mathrm{x})=\frac{\Delta \Phi_{0}}{\left(1+\mathrm{x}^{2}\right)}
$$

e

$$
\Delta \Phi_{0}=\mathrm{kL}_{\mathrm{eff}} \mathrm{n}_{2}\left(\frac{2 \mathrm{P}}{\pi \mathrm{w}_{0}^{2}}\right)
$$


pois $\Delta \phi_{0}(\mathrm{x})=\mathrm{KL}_{\mathrm{eft}} \mathrm{n}_{2}\left(2 \mathrm{P} / \pi \mathrm{w}^{2}\right)$, onde o termo entre parêntesis representa a intensidade axial a qual depende de $x$, uma vez que $w$ depende de $x$ de acordo com a Eq. (3.5). Se a amostra tem um coeficiente de absorção A não desprezivel, o efeito da absorção linear deve ser levado em consideração na Eq. (3.11), de tal forma que introduzindo uma espessura efetiva $\left(L_{\text {eff }}\right)$ da amostra dada por[19]:

$$
\mathrm{L}_{\mathrm{eff}}=\frac{1-\mathrm{e}^{-\mathrm{AL}}}{\mathrm{A}}
$$

a Eq. (3.11) continua válida.

A integral da Eq. (3.15) é tabelada e tem como solução:

$$
H=\frac{\exp \left[-\beta^{2} 4(1+i x)\right]}{2(1+i x)}
$$

Substituindo a Eq. (3.19) em (3.16) e resolvendo de maneira análoga a (3.15) temos como resultado:

$$
\delta H=-i \Delta \phi_{0}(x) \frac{\exp \left[-\beta^{2} / 4(1+i x)\right]}{2(1+i x)}
$$

De posse dos resultados de $\mathrm{H}$ e $\delta \mathrm{H}$ temos o perfil de intensidade $\mathrm{I}_{\mathrm{B}} \propto\left|\varepsilon_{\mathrm{B}}\left(\mathrm{r}_{1}\right)\right|^{2}$. Este resultado é igual ao derivado por Weaire et. al. usando o método da

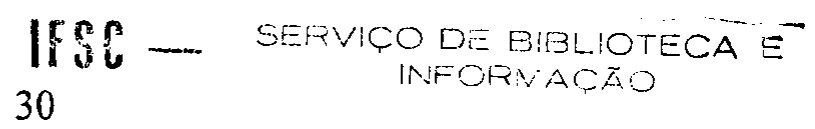


decomposição gaussiana, que é o tratamento utilizado pelos inventores da técnica Z-scan[17].

Aqui queremos apenas encontrar a expressão para a forma da curva de Z-scan e isto pode ser feito por analisar apenas o campo no centro $\left(r_{1}=0\right)$. Observe que neste caso temos $\beta=0$ e a função $\mathrm{J}_{0}(0)=1$, desta maneira as integrais (3.15) e (3.16) se tornam simples e temos como resultado:

$$
\begin{gathered}
H=\frac{1}{2(1+i x)} \\
\delta H=-i \Delta \phi_{0}(x) \frac{1}{2(1+i x)}
\end{gathered}
$$

Usando as Eqs. (3.18, 3.20, 3.24 e 3.25) teremos como expressão final:

$$
\mathrm{T}\left(\mathrm{x}, \Delta \Phi_{0}\right)=1+\frac{4 \mathrm{x} \Delta \Phi_{0}}{\left(9+\mathrm{x}^{2}\right)\left(1+\mathrm{x}^{2}\right)}
$$

Esta expressão tem a forma característica mostrada na Figura 3.5 com 2 extremos, um máximo (posição de pico) e um mínimo (posição de vale) dados por $z= \pm 0.858 z_{0}$, de tal forma que a separação entre o pico e o vale seja:

$$
\Delta \mathrm{z}_{\mathrm{pv}} \sim 1.7 \mathrm{z}_{0}
$$




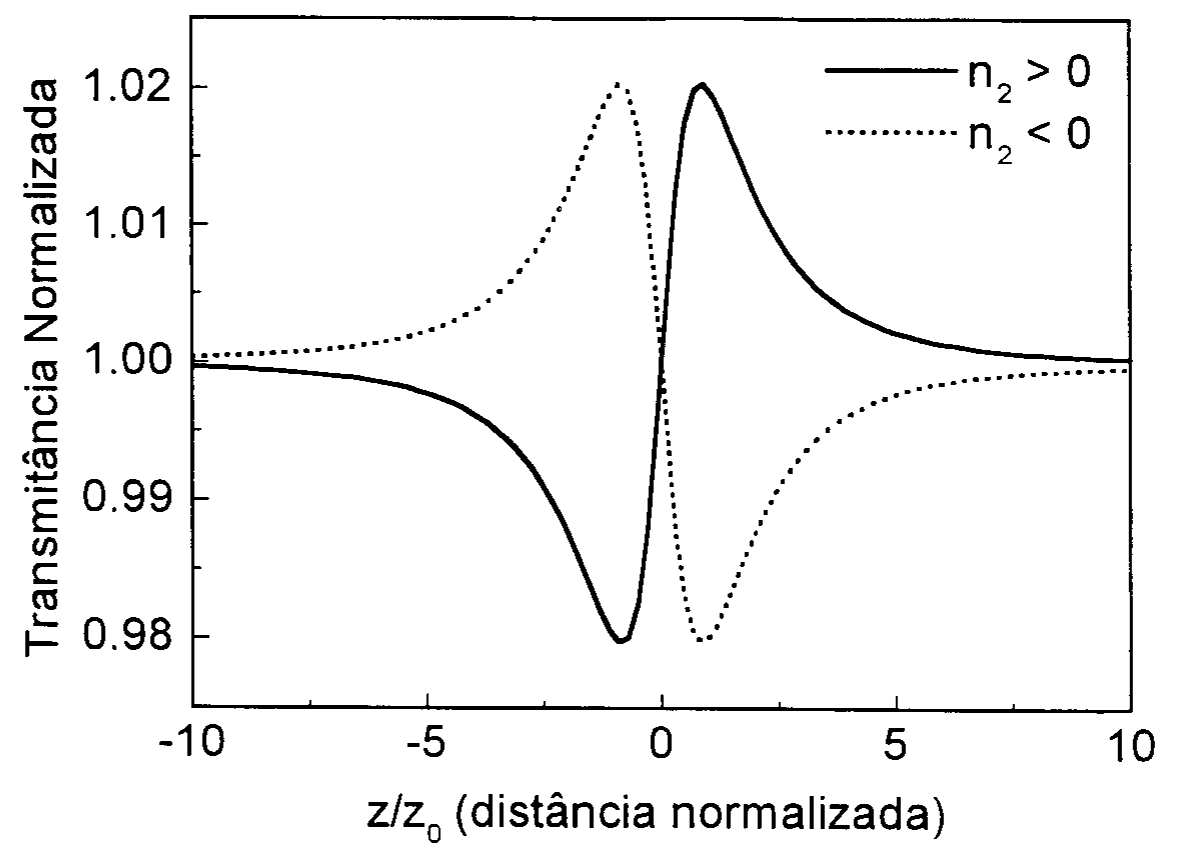

Figura 3.5- Curva característica da técnica de Z-scan com $n_{2}>0$ linha cheia e $\mathrm{n}_{2}<0$ linha pontilhada.

Lembrando que a medida é feita com uma íris frente ao detector, é necessário que se leve em consideração o tamanho da abertura com a qual é realizado o experimento. Logo, a potência não é medida somente em $r=0$, o que corresponde a utilizar o raio da abertura da íris $\left(\mathrm{r}_{\mathrm{a}}\right)$, infinitesimal quando comparada ao raio do feixe w. Do ponto de vista experimental, é importante considerar o caso em que o raio da abertura da íris $\left(r_{a}\right)$, e o tamanho do feixe sejam comparáveis, já que uma abertura muito pequena tende a ser muito sensível às flutuações do modo do laser e imperfeições na superficie do cristal. Logo, sendo w o valor da cintura do feixe no regime linear, a transmissão linear da abertura sem a amostra é dada por: $S=-\exp [-2$ $\left.\mathrm{r}_{\mathrm{a}}{ }^{2} / \mathrm{w}^{2}\left(\mathrm{x}_{\mathrm{B}}\right)\right]$. Baseando-se em resultados de simulações numéricas [4] verifica-se que quando o tamanho da abertura da íris é levado em consideração a expressão (3.26) precisa ser corrigida por um fator da abertura dado por $(1-\mathrm{S})^{0.25}$. Desta maneira a variação de transmissão entre o pico e o vale fica dado por: 


$$
\Delta \mathrm{T}_{\mathrm{pv}} \sim 0.406(1-\mathrm{S})^{0.25} \Delta \Phi_{\mathrm{o}}
$$

As Eqs. (3.28) e (3.29) são expressões fundamentais na determinação de $n_{2}$ de uma medida de Z-scan. Através dos dados experimentais de T(z) usa-se a variação de transmissão $\Delta \mathrm{T}_{\mathrm{pv}}$ (pico-vale) para encontrar $\Delta \Phi_{0}$. De posse desse dado, usa-se a Eq.(3.21) para calcular $n_{2}$. O valor de $w_{0}{ }^{2}$ pode ser encontrado através de $z_{0}$ obtido da Eq. (3.28).

O tratamento teórico da técnica de Z-scan pode ser generalizado para o caso onde se trabalha com dois feixe, um feixe de prova $\left(w_{p}\right)$ e um feixe de excitação $\left(w_{e}\right)$ que apresentam diâmetros diferentes. Logo, o perfil de fase dado pela Eq. (3.19) será:

$$
\Delta \phi(\mathrm{y}, \mathrm{x})=\Delta \phi_{0}(\mathrm{x}) \exp \left[-2 \mathrm{my}^{2}\right]
$$

onde $\mathrm{m}=\left(\mathrm{w}_{\mathrm{p}} / \mathrm{w}_{\mathrm{e}}\right)^{2}$ é a razão entre os dois feixes, como é definido na Técnica de Lente Térmica que trataremos a seguir.

Seguindo o mesmo procedimento utilizado para obter a Eq.(3.27), teremos como expressão final (veja Apêndice A):

$$
\mathrm{T}\left(\mathrm{x}, \Delta \Phi_{0}\right)=1+\frac{4 \mathrm{mx} \Delta \Phi_{0}}{\left(1+4 \mathrm{~m}^{2}+4 \mathrm{~m}+\mathrm{x}^{2}\right)\left(1+\mathrm{x}^{2}\right)}
$$




\subsection{Absorção não linear}

Em grande parte dos materiais que apresentam um grande valor de $n_{2}$ de origem eletrônica, existe também o efeito de absorção não-linear. Neste caso a absorção varia com a intensidade devido, por exemplo, ao fenômeno de dois fótons ou absorção do estado excitado. Neste caso a intensidade dentro da amostra se atenua seguindo a seguinte equação:

$$
\frac{\mathrm{dI}}{\mathrm{d} z^{\prime}}=-\mathrm{AI}-\mathrm{BI}^{2}
$$

onde z' é a variável de integração dentro da amostra, logo z' varia entre 0 e L. B é o coeficiente de absorção não-linear.

Considerando o caso geral onde $\mathbf{n}_{2}$ é complexo, sua parte imaginária $\mathbf{n}_{2}$ " será proporcional a $B=4 \pi n_{2} " / \lambda$, onde $n_{2}$ " é dado pela Eq. (2.17). No caso $\sigma_{\mathrm{Ex}}=0, \mathrm{~B}$ representa apenas a saturação da absorção. Em geral quando $\Delta \sigma<0$ o sistema se comporta como um absorvedor saturável (a absorção diminui com a intensidade) e quando $\Delta \sigma>0$ o sistema é um absorvedor reverso saturável (a absorção aumenta com a intensidade).

Em outros sistemas, por exemplo em semicondutores excitado por lasers pulsados de alta potência, o fator B é usado para representar o efeito de absorção de dois fótons. Entretanto, para os nossos sistemas o efeito de absorção de dois fótons é desprezivel pois trabalhamos com lasers contínuos (cw) com baixas potências $(\sim W)$, pequenas comparadas às potência de pico de lasers pulsados $(\sim \mathrm{GW})$. 
Integrando a Eq. (3.32) em z', de 0 a L, pode-se determinar a intensidade média na amostra[19]:

$$
\dot{I}\left(r_{0}, z\right)=\frac{\ln \left[1+\mathrm{BL}_{\mathrm{eff}} \mathrm{I}\left(\mathrm{r}_{0}, \mathrm{z}\right)\right]}{\mathrm{BL}}
$$

Neste caso a variação de fase $\Delta \phi\left(\mathrm{r}_{0}\right)$ será:

$$
\Delta \phi\left(\mathrm{r}_{0}\right)=\frac{\mathrm{kn}_{2}}{\mathrm{~B}} \ln \left[\mathrm{l}+\mathrm{BI}\left(\mathrm{r}_{0}, \mathrm{z}\right) \mathrm{L}_{\mathrm{eff}}\right]
$$

Expandindo o fator logarítmico na Eq. (3.34) e mantendo apenas o termo linear na intensidade obtém-se uma expressão idêntica à Eq. (3.19), logo em primeira aproximação a Eq. (3.34) não depende de B.

A amplitude do campo elétrico no plano de saída da amostra(Eq. (3.8)) é dada por[19]:

$$
\varepsilon_{\mathrm{A}}^{\prime}\left(\mathrm{r}_{0}\right)=\varepsilon_{0} \exp [-\mathrm{AL} / 2]\left[1+\left(\mathrm{ikn} n_{2}-\mathrm{B} / 2\right) \mathrm{I}\left(\mathrm{r}_{0}, \mathrm{z}\right) \mathrm{L}_{\mathrm{eff}}\right]
$$

que pode ser pensado como uma superposição de dois campos gaussianos. A expressão da transmitância normalizada para o caso de uma abertura da íris muito pequena é dada por: 


$$
\mathrm{T}(\mathrm{z})=\mathrm{T}\left(\mathrm{x}, \Delta \Phi_{0}\right)-\frac{2 \Delta \mathrm{A}_{0}\left[3+\mathrm{x}^{2}\right]}{\left[1+\mathrm{x}^{2}\right]\left[9+\mathrm{x}^{2}\right]}
$$

onde

$$
\Delta \mathrm{A}_{0}=\mathrm{BI}_{0} \frac{\mathrm{L}_{\mathrm{eff}}}{2}
$$

e $\mathrm{T}\left(\mathrm{x}, \Delta \Phi_{0}\right)$ é a transmitância normalizada definida na Eq. (3.27). $\mathrm{O}$ coeficiente $\Delta \mathrm{A}_{0}$ da uma idéia da fração de potência absorvida por processos de dois fótons. A Fig. 3.6 mostra a transmitância para os seguintes casos:

1. não linearidade puramente absorptiva, $\Delta \Phi_{0}=0$, veja segundo termo da Eq. (3.27).

2. não linearidade puramente refrativa, $B=0$.

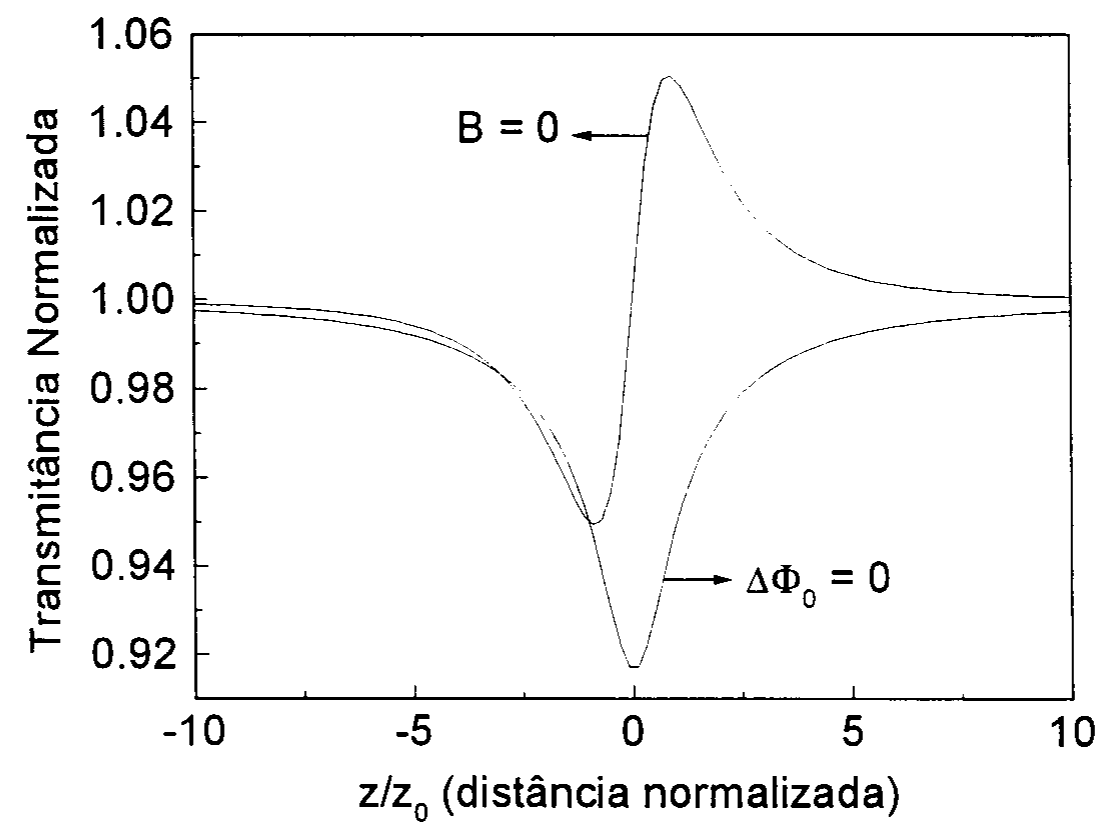

Figura 3.6- Transmitância normalizada em função da posição da amostra, nos casos puramente refrativo e no caso puramente absorptivo. 
Podemos notar da curva que, quando temos uma não linearidade puramente absoptiva, a transmitância diminui quando a amostra se aproxima do ponto focal, onde a absorção é maior.

Realizando uma medida com a íris totalmente aberta, podemos obter o valor do coeficiente não-linear $B$ e do parâmetro $z_{0}$, uma vez que a varredura será insensível a variações da fase e apresentará um mínimo na posição do foco (no caso $\left.\Delta \mathrm{A}_{0}>0\right)$ devido apenas a absorção não linear. Fazendo a integral radial $\mathrm{d} \epsilon\left|\varepsilon_{\mathrm{A}}{ }^{\prime}\right|^{2}$ (Eq. (3.35)), pode-se mostrar que a potência detectada após a amostra é dada por:

$$
P(z)=P_{i}\left(1-\frac{\Delta A_{0}}{\left(1+x^{2}\right)}\right) \exp [-A L]
$$

onde $P_{i}$ é a potência incidente na amostra. Obtidos os valores de $z_{0}$ e $\beta$ pode-se estimar o valor do índice de refração não-linear através da Eq. (3.36).

A Fig. 3.7 mostra que realizando uma medida com a iris $S<1$ e normalizando pela medida com $S=1$, permite dentro de uma incerteza de $\pm 10 \%$, uma curva de Z-scan livre da contribuição do efeito de absorção não linear. 


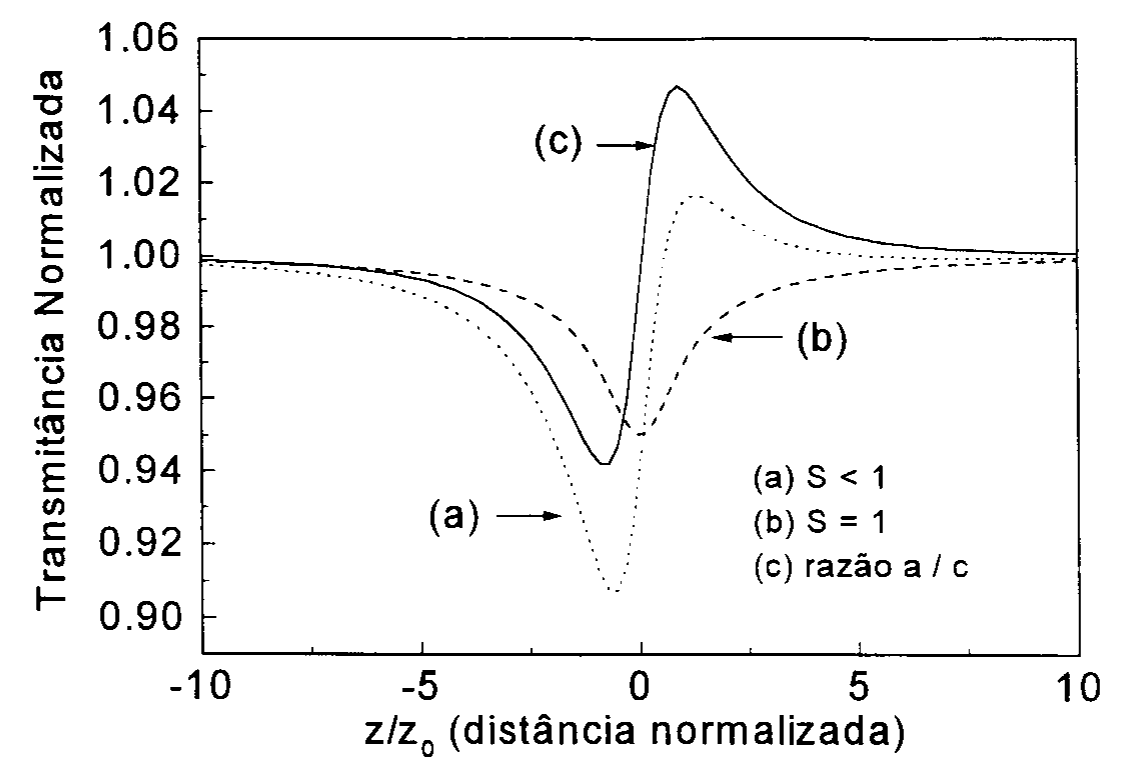

Figura 3.7- Simulação teórica da varredura $Z$ para o caso de amostras que apresentam absorção de dois fótons. (a) medida com abertura da íris $\mathrm{S}<1$, (b) medida com a íris totalmente aberta $(S=1)$ e $(c)$ razão entre as curvas a / c.

\subsection{Z-scan no caso de não-linearidade saturável}

A técnica de Z-scan no caso de não-linearidade saturável necessita de uma correção do fator de saturação, uma vez que, os resultados obtidos analiticamente por Sheik-Bahae et. al [4] são válidos somente para perfis de fase $\Delta \phi(y)$ gaussiano (Eq. 3.19). Porém o formalismo baseado na integral de difração de Kirchhoff em coordenadas cilíndricas, se transforma numa transformada de Hankel de ordem zero, podendo ser utilizada para incluir o efeito de saturação[10, 13].

$\mathrm{Na}$ ótica não-linear é muito importante o caso de não-linearidade saturável, onde o perfil de índice de refração não é gaussiano porque o índice de refração é dado por: 


$$
\mathrm{n}=\mathrm{n}_{0}+\frac{\mathrm{n}_{2} \mathrm{I}}{1+\mathrm{I} / \mathrm{I}_{\mathrm{s}}}
$$

onde $\mathbf{I}_{\mathrm{s}}$ é a intensidade de saturação. Para $\mathbf{I}<<\mathbf{I}_{\mathrm{s}}$ o perfil de população do estado excitado é gaussiano e tende a mesma forma do perfil de intensidade do laser; o perfil de índice de refração também é gaussiano e a teoria de Sheik-Bahae et. al. pode ser aplicada. Porém, quando $\mathrm{I} \sim \mathrm{I}_{\mathrm{s}} \mathrm{o}$ perfil de índice de refração deixa de ser gaussiano e a teoria de Sheik-Bahae deixa de ser aplicável. Através de cálculos numéricos obteve-se uma expressão empírica relacionado $\Delta \mathrm{T}_{\mathrm{pv}}$ a $\Delta \phi_{0}$ e $\mathrm{I}_{0} / \mathrm{I}_{\mathrm{s}}[10]$ :

$$
\Delta \mathrm{T}_{\mathrm{pv}} \approx 0.406(1-\mathrm{S})^{0.25\left(1+1_{0} / \mathrm{s}_{\mathrm{s}}\right)^{(1-s)}} \frac{\left|\Delta \Phi_{0}\right|}{\left(1+\mathrm{I}_{0} / \mathrm{I}_{\mathrm{s}}\right)^{0.525}}
$$

onde $\Delta \Phi_{0}$, definido em (3.21), é o valor máximo do perfil de fase no caso não saturado. Foi obtido também uma expressão empírica para as medidas de absorção não-linear com a abertura da íris totalmente aberta $(\mathrm{S}=1)$ :

$$
\mathrm{T}_{\mathrm{p}}-\mathrm{l} \approx \frac{2 \pi}{\lambda} \mathrm{n}_{2}^{\prime \prime} \mathrm{L}_{\mathrm{eff}} \frac{\mathrm{I}_{0}}{\left(1+\mathrm{I}_{0} / \mathrm{I}_{\mathrm{s}}\right)^{0.76}}
$$

onde $T_{p}$ é a transmissão normalizada em $z=0$. 


\subsection{Técnica de Lente Térmica}

$\mathrm{Na}$ maioria dos materiais para laser um efeito extremamente importante é a variação de índice de refração induzida pelo laser através do chamado efeito térmico. Neste caso, a absorção de luz na amostra gera uma distribuição de temperatura. Quando um laser contínuo incide na amostra, ela vai esquentando e ao mesmo tempo o calor vai se difundindo radialmente (no caso de excitação por um feixe gaussiano TEM $_{\mathrm{oo}}$ ) tal como mostra a Fig. 3.8.

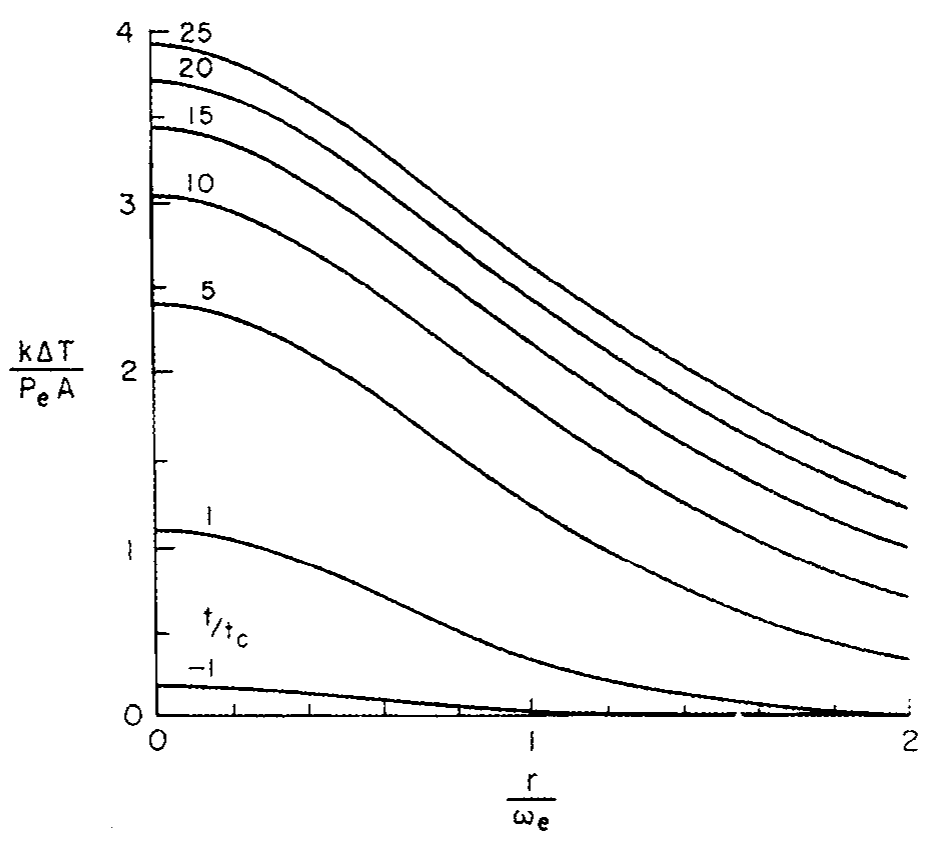

Figura 3.8- Distribuição de temperatura $\Delta T(r)$ na Lente Térmica em função do tempo[20]

O efeito de lente é causado pela deposição de calor por um processo de decaimento não-radiativo após a luz do laser ter sido absorvida pela amostra. Ao passar pela amostra, a radiação laser ao ser absorvida e convertida em calor provocará uma mudança no índice de refração da amostra com um perfil gaussiano, gerando assim o efeito de Lente Térmica (LT). Pela determinação da intensidade no centro do feixe num campo longe, as propriedades termo-ópticas podem ser 
determinadas. O método resolvido no tempo, permite medidas do desenvolvimento da Lente Térmica em um curto tempo, da ordem de milisegundos, que é o tempo necessário para que o equilíbrio térmico ocorra, ou seja, o perfil de temperatura $\Delta \mathrm{T}(\mathrm{r})$ atinja o estado estacionário. Neste caso a difusividade térmica pode ser determinada. $\mathrm{Na}$ grande maioria dos líquidos, o índice de refração diminui com a temperatura por causa da diminuição da intensidade na região iluminada pela luz do laser. Neste caso, teremos $\mathrm{dn} / \mathrm{dT}$ negativo e a lente provocará uma divergência do feixe laser. Em sólidos, dependendo da composição da amostra, dn/dT pode ser negativo ou positivo No caso de amostras que apresenta alta mudança de polarizabilidade com a temperatura, $\mathrm{dn} / \mathrm{dT}$ tende a ser positivo e assim o feixe laser é convergido após passar pela amostra.

O método de feixe duplo, configuração descasada, desenvolvido recentemente é mais sensivo do que o método de feixe duplo, configuração casada, ou simplesmente feixe simples[3]. No arranjo experimental para a configuração descasada mostrada na Fig. 3.9 um feixe Gaussiano cw TEM 00 , ilumina a amostra, gerando o efeito de Lente Térmica (LT). Um fraco feixe de prova Gaussiano TEM $_{00}$, passa através da amostra com a finalidade de investigar a Lente Térmica. A posição da cintura do feixe de prova está localizado na origem ao longo do eixo $z$. A amostra é localizada em $z$ e o plano do detector se encontra em $z+d$. A cintura do feixe de prova é $w_{0 p}$, o raio do feixe de prova e o feixe de excitação na amostra são $w_{p}$ e $w_{e}$, respectivamente. A partir da utilização de um pequeno orificio posicionado na frente do detector do feixe de prova, a intensidade do centro do feixe $\left(r_{1}=0\right)$ pode ser monitorada e associada à absorção do material, permitindo determinação quantitativa de suas propriedades ópticas e térmicas: difusividade, variação do caminho óptico 


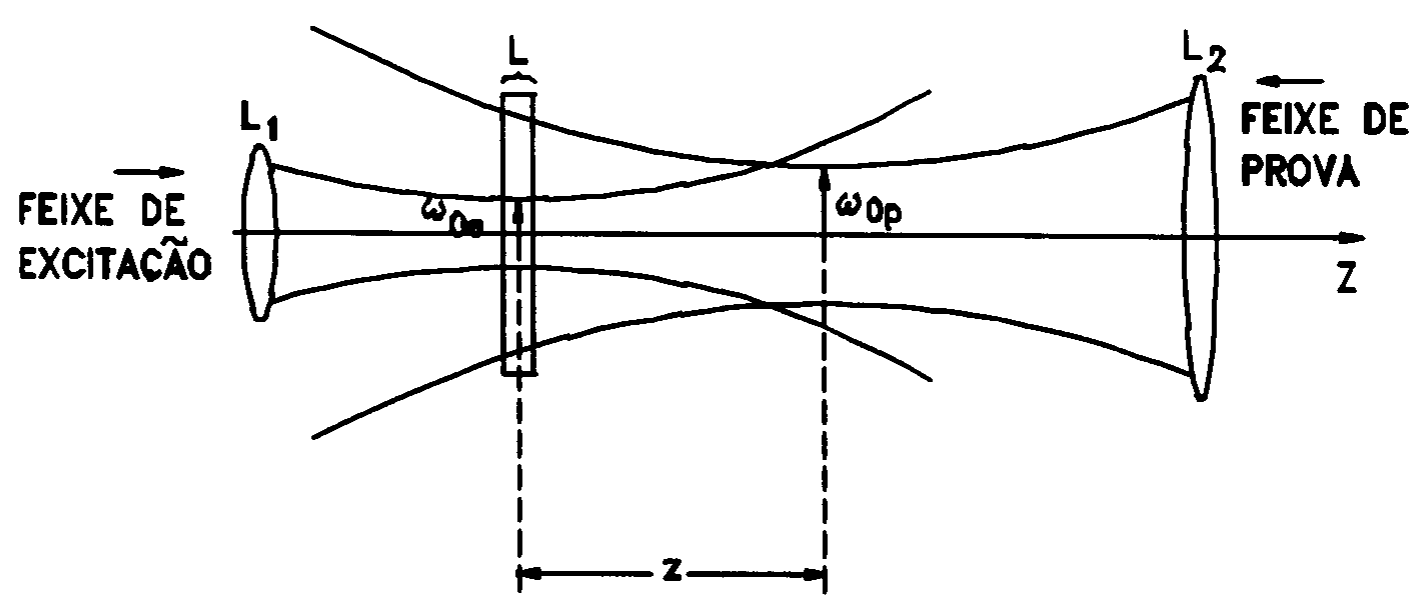

Figura 3.9- Arranjo dos feixes de excitação e prova na configuração do experimento de Lente Térmica com feixe duplo de modo descasado, onde $\mathrm{L}_{1} \mathrm{e}$ $\mathrm{L}_{2}$ são as lentes e $\mathrm{w}_{0 \mathrm{e}} \mathrm{e} \mathrm{w}_{0 \mathrm{p}} \mathrm{o}$ raios dos feixes de excitação e prova respectivamente.

Esta técnica tem sido utilizada com sucesso em estudos de soluções com concentração de impurezas muito baixa. O limite de deteç̧ão alcançado para o coeficiente de absorção óptica é de $10^{-7} \mathrm{~cm}^{-1}$. A configuração utilizada para a técnica de Lente Térmica (LT) no modo descasado consiste de dois feixes lasers, um para excitação tendo a cintura na amostra, e outro de prova tendo a amostra na sua posição confocal, o qual é afetado pela lente térmica criada em função da absorção da de luz com geração de calor na amostra. $O$ ângulo de inclinação entre os dois feixes, uma inovação recente no sistema, facilita o alinhamento e reduz a quantidade de elementos ópticos na montagem. Além disso, a utilização de filtros na frente do detector elimina sinais indesejáveis do laser de excitação no fotodiodo. Esta configuração é a mais sensível já encontrada, e só começou a ser utilizada em estudos quantitativos de sólidos, vidros e polímeros, nos últimos anos 
No experimento de Lente Térmica, a variação de temperatura na amostra, provoca uma mudança de fase do laser ao passar pela lente térmica dada por $\Delta \phi(r) \propto$ $\Delta \mathrm{T}(\mathrm{r})$. Embora no caso térmico a expressão para $\Delta \phi(\mathrm{r})$ seja mais complexa, o procedimento do cálculo é análogo ao feito para o caso do Z-scan (seções 3.3, 3.4 e 3.5). Para tal supõe-se que $\Delta \phi(r)<<1$ e resolve-se a Eq. (3.16) apenas para o centro do feixe (caso particular $r_{1}=0$ ). A variação da intensidade no centro do feixe de prova pode ser expressa como[18]:

$$
I(t)=I(0)\left\{1-\frac{\theta}{2} \operatorname{atan}\left[\frac{2 m x}{\left[(1+2 m)^{2}+x^{2}\right] \frac{t_{c}}{2 t}+1+2 m+x^{2}}\right]\right\}^{2}
$$

onde

$$
\mathrm{m}=\left(\frac{\mathrm{w}_{\mathrm{p}}}{\mathrm{w}_{\mathrm{e}}}\right)^{2}
$$

$$
\mathbf{x}=\mathbf{z} \mathbf{z}_{0}
$$

onde $\mathrm{I}(0)$ é o valor de $\mathrm{I}(\mathrm{t})$ quando $\mathrm{t}$ ou $\theta$ é zero.

$$
t_{c}=\frac{w_{e}^{2}}{4 D}
$$

onde $\mathrm{D}=\mathrm{k} / \rho \mathrm{C}$, é a difusividade térmica $\left(\mathrm{cm}^{2} / \mathrm{s}\right), \mathrm{k}$ é a condutividade térmica $\left(\mathrm{Js}^{-1} \mathrm{~cm}^{-1} \mathrm{~K}\right), \rho$ é a densidade $\left(\mathrm{gcm}^{-3}\right), \mathrm{C}$ é 0 calor específico $\left(\mathrm{Jg}^{-1} \mathrm{~K}^{-1}\right), \mathrm{t}_{\mathrm{c}}$ é a constante 
característica tempo térmico, $\theta$ é a diferença de fase do laser de prova em $\mathrm{r}=0 \mathrm{e}$ $\mathrm{r}=1.44 \mathrm{w}_{\mathrm{e}}$, induzida pela lente térmica.

$$
\theta=\frac{P_{e} A L_{0}}{K \lambda_{p}} \frac{d s}{d T}
$$

onde $\mathrm{P}_{\mathrm{e}}$ é a potência do laser de excitação $(\mathrm{W})$, A é o coeficiente de absorção $\left(\mathrm{cm}^{-1}\right)$, $\mathrm{L}$ é a espessura da amostra $(\mathrm{cm}), \lambda_{\mathrm{p}}$ é o comprimento de onda do feixe de prova $(\mathrm{cm})$. Medindo-se portanto, a variação da intensidade transiente do laser de prova $I(t)$ e ajustando o resultado experimental através da Eq. (3.42) obtém-se os valores de $\theta$ e a difusividade térmica D. A variação da parte óptica com a temperatura ds/dT, pode ser analisada a partir da equação:

$$
\frac{\mathrm{ds}}{\mathrm{dT}}=(\mathrm{n}-1)(1+v) \alpha+\frac{\left(\mathrm{n}^{2}-1\right)\left(\mathrm{n}^{2}+2\right)}{6 \mathrm{n}}(\phi-\beta)
$$

onde a segunda parte da equação é o $\mathrm{dn} / \mathrm{dT}$, variação do índice de refração com a temperatura, $\alpha$ e $\beta$ são respectivamente o coeficiente de expansão térmica linear e volumétrico, $\phi$ é o coeficiente de temperatura da polarizabilidade eletrônica e $v$ é o coeficiente de Poisson.

A Eq. (3.42) também é válida para o caso em que se trabalha com um único feixe de laser, o qual faz simultaneamente o papel de feixe de excitação e de prova, tal como no experimento de Z-scan convencional. Neste caso tem-se $\mathrm{m}=1$, ou seja $\left(w_{e}=w_{p}\right)$. Note que a sensibilidade da técnica de Lente Térmica depende dos 
parâmetros m e $x$ e que o sinal $\Delta T \propto(I(t)-I(0))$ é nulo em $x=0$ e a curva $\Delta T(x)$ tem uma forma parecida com a do Z-scan na Fig. 3.5. Assim a configuração com dois feixes é utilizada, uma vez que a sensibilidade aumenta com o aumento do parâmetro m.

Existe uma diferença importante entre o efeito não-linear e o efeito térmico. No caso não-linear a perturbação $\Delta \mathrm{n}(\mathrm{r})$ é localizada enquanto que no caso térmico ela se expande até valores de $r$ bem maiores que $w$, devido a difusão de calor. Consequentemente, no caso não-linear a sensibilidade diminui se m aumenta (Eq. (3.31)) muito, enquanto que no caso térmico a sensibilidade aumenta com m (Eq. (3.42)). 


\section{CAPÍTULO 4}

\section{RESULTADOS EXPERIMENTAIS}


Neste capítulo, apresentaremos resultados em sólidos dopados com ions $\mathrm{Nd}^{+3}$ e $\mathrm{Cr}^{+3}$, com o uso das técnicas de Lente Térmica e Z-scan. Estas técnicas se mostraram eficientes no estudo de efeitos não-lineares e térmicos mesmo apresentando simplicidade na montagem experimental. Na tabela abaixo temos a composição dos vidros estudados neste trabalho.

\begin{tabular}{|c|c|c|c|c|c|c|c|}
\hline AMOSTRA & \multicolumn{7}{|c|}{ COMPOSIÇÃO (\%) } \\
\hline $\begin{array}{c}\text { Aluminato } \\
\text { de cálcio }\end{array}$ & $\begin{array}{c}41.5 \\
\mathrm{AlO}_{3}\end{array}$ & $\begin{array}{c}47.4 \\
\mathrm{CaO}\end{array}$ & $\begin{array}{c}7 \\
\mathrm{SiO}_{2}\end{array}$ & $\begin{array}{c}4.1 \\
\mathrm{MgO}\end{array}$ & $\begin{array}{c}\mathrm{x}^{*} \\
\mathrm{Nd}_{2} \mathrm{O}_{3}\end{array}$ & & \\
\hline ZBLAN & 53 & 4.5 & 3.5 & 29 & 10 & 1 & \\
& $\begin{array}{c}\mathrm{ZrF}_{4} \\
\mathrm{LaF}_{3}\end{array}$ & $\mathrm{AlF}_{3}$ & $\begin{array}{c}\mathrm{BaF}_{2} \\
\mathrm{NaF}\end{array}$ & $\mathrm{NdF}_{3}$ & \\
\hline InSBZnGdN & 2 & 40 & 20 & 16 & 20 & 2 & 1 \\
& $\begin{array}{c}2 \\
\mathrm{GdF}_{3}\end{array}$ & $\mathrm{InF}_{3}$ & $\mathrm{SrF}_{2}$ & $\begin{array}{c}\mathrm{BaF}_{2} \\
\mathrm{ZnF}_{2}\end{array}$ & $\begin{array}{c}\mathrm{NaF} \\
\mathrm{NdF}_{3}\end{array}$ \\
\hline
\end{tabular}

Tabela 4.1- Tabela com a composição das amostras utilizadas neste trabalho.

* Para o vidro aluminato tínhamos as seguintes concentrações: $(0.5,1.0,2.0$, $2.5,3.0,3.5,4.0,4.5$ e 5.0$) \%$.

\subsection{Medidas de Lente Térmica}

\subsubsection{Montagem experimental}

Realizamos medidas de Lente Térmica com duplo feixe na configuração descasada. Esta configuração apresenta a vantagem de ser mais sensível do que a configuração de modo casada (ou feixe simples). A montagem experimental que utilizamos está esquematizada na Fig. 4.1. A vantagem, no caso da configuração de modo descasado, é que a sensibilidade pode ser significativamente aumentada com o uso de um feixe de prova com cintura $\left(w_{0 p}\right)$ maior do que a cintura do feixe de excitação $\left(\mathrm{w}_{\mathrm{e}}\right)$. Este aumento na sensibilidade é uma conseqüência da difusão de calor 
que faz com que o perfil do índice de refração $\Delta \mathrm{n}(\mathrm{r})$ seja maior do que o perfil de intensidade do feixe de excitação. Porém, no efeito de LP não existe a presença do efeito de difusão. Assim sendo, o efeito de LP decresce com o aumento da cintura do feixe de prova quando $w_{p}>w_{e}$. No Capítulo 3 definimos o parâmetro $m=\left(w_{p} / w_{e}\right)^{2}$, e com isto é possível mostrar que o sinal de LP diminui aproximadamente com $1 / \mathrm{m}$, para $m>>1$. Esta é uma vantagem adicional na configuração descasada, quando os efeitos de LP e LT aparecem simultaneamente. Através do aumento do fator m podese eliminar o efeito de LP e apenas o efeito de LT aparece possibilitando a determinação dos parâmetros D (difusividade térmica) e ds/dT (variação do caminho óptico com a temperatura)

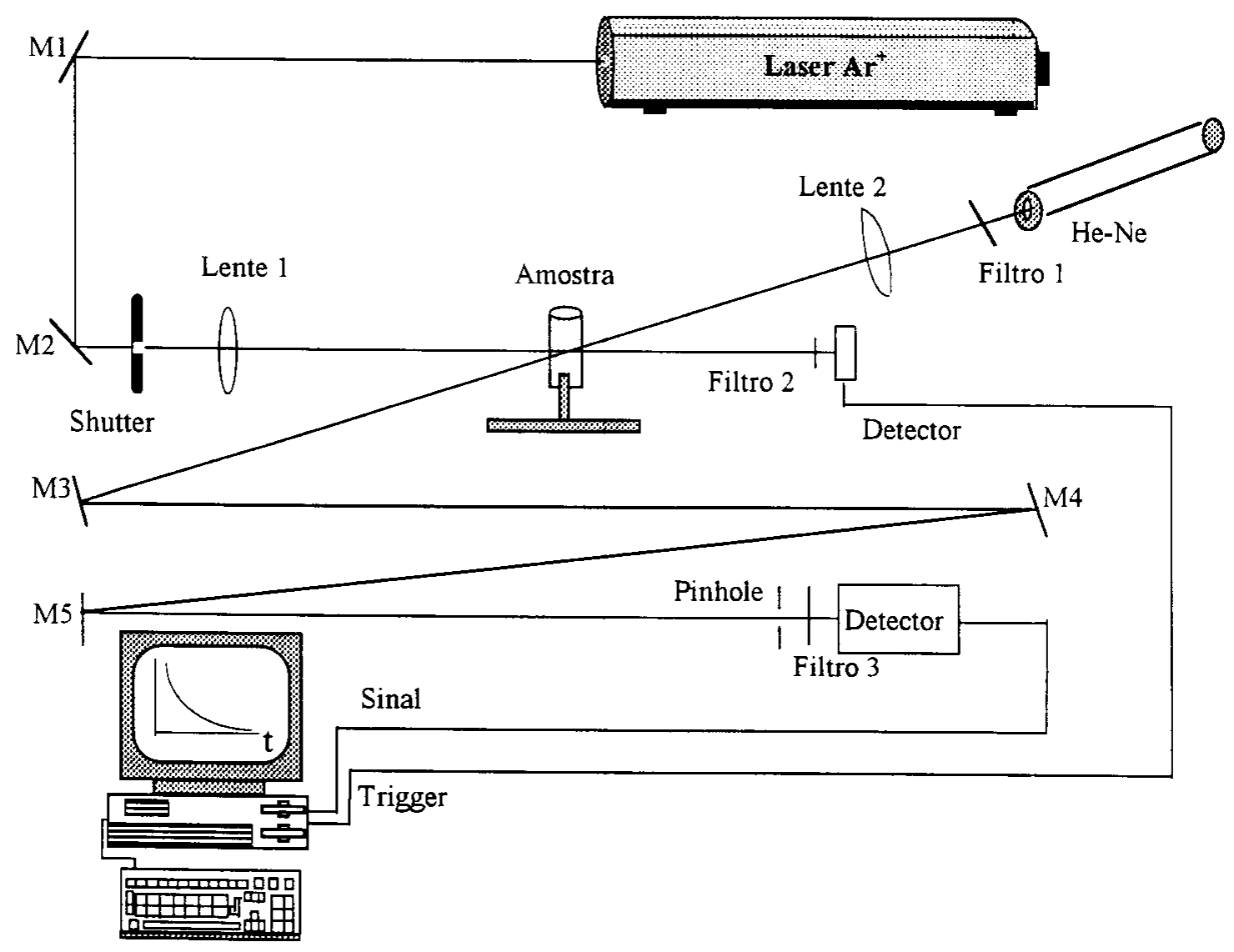

Figura 4.1- Aparato experimental do experimento de LT. M1, M2, M3, M4 e M5 são os espelhos. 
A obtenção dos parâmetros necessários às medidas de lente térmica requerem um procedimento habitual para o alinhamento dos feixes lasers utilizados no aparato experimental. Neste procedimento o feixe do laser de excitação foi focalizado por uma lente convergente de (Lente 1) $f_{1}=20 \mathrm{~cm}$, enquanto que, para o feixe do laser de prova uma lente convergente de (Lente 2) $f_{2}=15 \mathrm{~cm}$. A obtenção da posição das cinturas dos lasers de excitação e de prova, foi feita através de uma varredura da potência do laser sobre uma régua, utilizando-s s um fotodiodo (contendo um pinhole) como medidor de potência. O pinhole, colocado na frente do fotodiodo, permite que apenas o centro do feixe do laser seja detectado possibilitando, desta forma, captar a variação da intensidade ao longo da régua.

Lembrando que $I_{0}=2 P / \pi w^{2}(x)$, onde $w^{2}(x)$ é dado pela Eq. (3.5), podemos ajustar os dados obtidos na varredura e determinar o parâmetro $\mathrm{z}_{0}$. Uma vez encontrado $z_{0}$, podemos encontrar a cintura do feixe $w_{0}$.

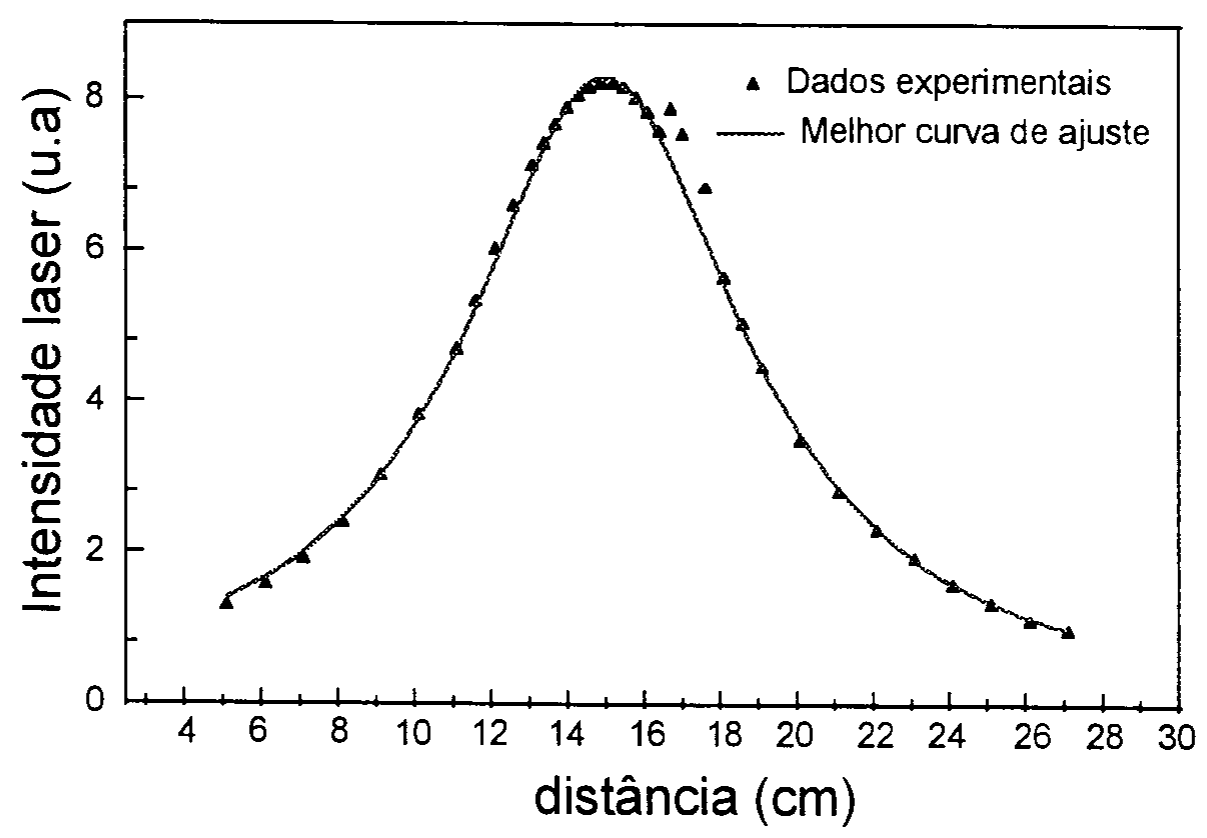

Figura 4.2- Posição da cintura do laser de $\mathrm{He}-\mathrm{Ne}, \lambda=632.8 \mathrm{~nm}$. 


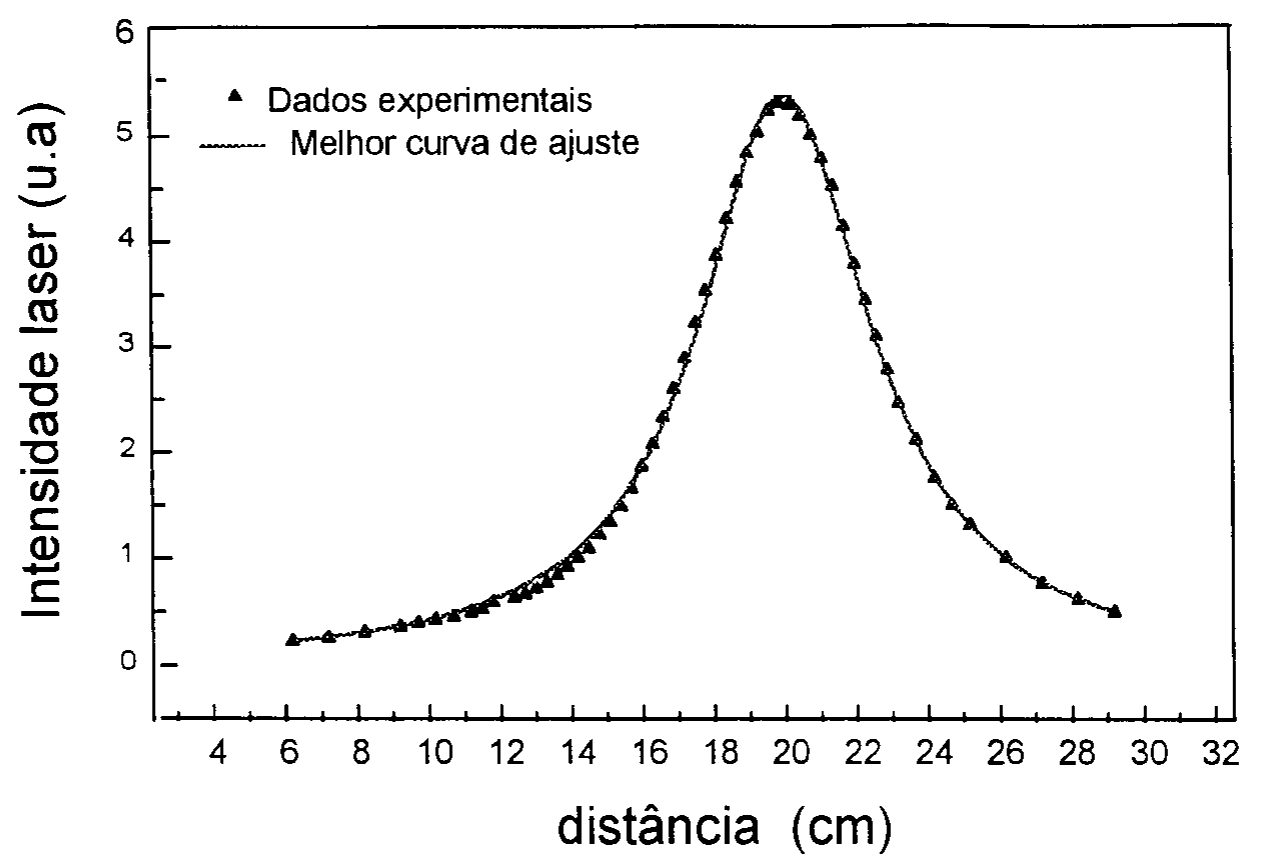

Figura 4.3- Posição da cintura do laser $\mathrm{Ar}, \lambda=514.5 \mathrm{~nm}$.

\begin{tabular}{|c|c|c|c|}
\hline Laser & $\lambda(\mathbf{n m})$ & $\mathbf{z}_{0}(\mathbf{c m})$ & $\mathbf{w}_{\mathbf{0}}(\mathbf{c m})$ \\
\hline $\mathrm{He}-\mathrm{Ne}$ & 632.8 & 2.5 & $7.1 \times 10^{-3}$ \\
\hline $\mathrm{Ar}$ & 514.5 & 2.6 & $6.5 \times 10^{-3}$ \\
\hline
\end{tabular}

Tabela 4.2- Valores dos parâmetros obtidos.

Em nossas medidas, a amostra foi colocada no foco do laser de excitação e ligeiramente deslocada do foco do laser de prova $(z=8 \mathrm{~cm})$, possibilitando desta forma um aumento na sensibilidade do experimento, uma vez que a sensibilidade dependo do parâmetro m, Eq. (3.43). Em nossa configuração, o diâmetro do feixe de prova na amostra era $\mathrm{W}_{\mathrm{p}}=23.5 \times 10^{-3} \mathrm{~cm}$ e com os parâmetros $\mathrm{x}=3.15 \mathrm{e} \mathrm{m}=13$.

Estes parâmetros, quando introduzidos na Eq. (3.42), são mantidos fixos, enquanto que os parâmetros $\theta$ e $t_{c}$ são variáveis. Fazendo-se iterações numéricas com a ajuda de um programa de computador, consegue-se ajustar a curva teórica aos dados experimentais. Os parâmetros $\theta$ e $t_{c}$ possuem informações das propriedades 
termo-ópticas da amostra, isto é, difusividade térmica e mudança do caminho óptico, ds/dT, induzida pela variação da temperatura.

\subsubsection{Resultados}

Em nossos experimentos utilizamos um laser de $\mathrm{Ar}^{+}$como feixe de excitação e medimos a variação de fase induzida com um laser de HelJe $(\lambda=633 \mathrm{~nm})$ como feixe de prova. Nós medimos a evolução do sinal do feixe de prova, como mostra a Fig. 4.4. O ajuste teórico foi feito com a expressão (3.42). Deste ajuste teórico, tiramos $\theta=-0.089$ e $t_{c}=1.8 \mathrm{~ms}$. A difusividade térmica que está relacionada a $t_{c}$ pode ser obtida através da Eq. 3.43 .

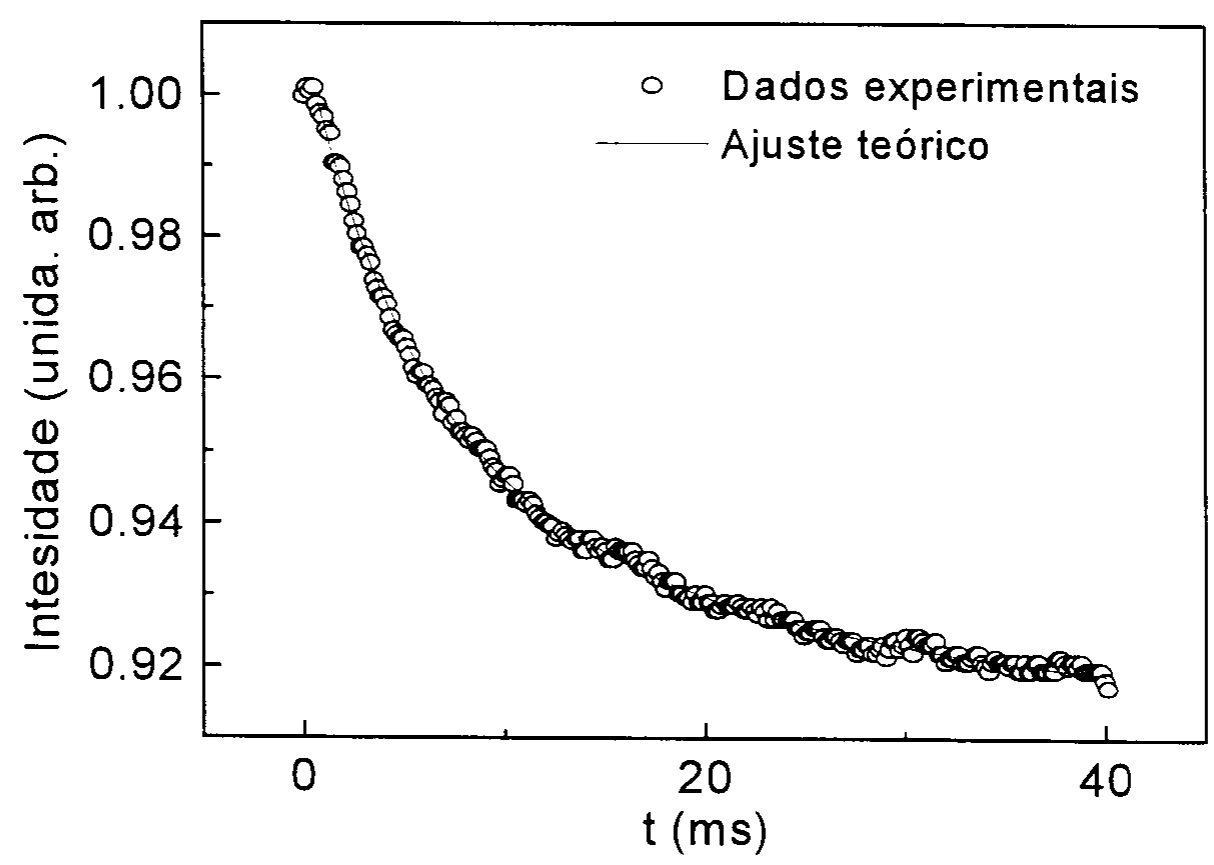

Figura 4.4- Desenvolvimento do sinal do feixe de prova na amostra $\mathrm{SrAlF}_{5}: \mathrm{Cr}^{+3}$ com potência do laser $(\mathrm{P}=600 \mathrm{~mW})$. Com o ajuste teórico obtivemos $\theta=-0.089$ e $t_{c}=1.8 \mathrm{~ms}$. 
Realizamos ainda medidas em outras amostras, vidros fluorindatos dopados com $\mathrm{Nd}^{+3}$ e com $\mathrm{Er}^{+3}$ e vidros de aluminato de cálcio puro e dopados com $\mathrm{Nd}^{+3}$, cujos resultados da difusividade estão apresentados na Tabela 4.3.

\begin{tabular}{|c|c|}
\hline Amostra & D $\left(10^{-3} \mathrm{~cm}^{2} / \mathrm{s}\right)$ \\
\hline $\mathrm{SrAlF}_{5}: \mathrm{Cr}^{+3} \mathrm{E} \perp \mathrm{c}$ & 6.5 \\
\hline $\mathrm{SrAlF}_{5}: \mathrm{Cr}^{+3} \mathrm{E} / / \mathrm{c}$ & 6.4 \\
\hline $\mathrm{LiKYF}_{5}: \mathrm{Nd}$ & 6.0 \\
\hline vidro InSBZnGdN:Nd & 4.0 \\
\hline vidro fluorindato: $\mathrm{Er}$ & 2.0 \\
\hline vidro aluminato de cálcio: $\mathrm{Nd}$ & $5.5 *$ \\
\hline
\end{tabular}

Tabela 4.3- Valores de difusividade térmica obtidos pelo método de lente térmica no modo descasado.

* valor médio para várias amostras com concentrações diferentes de $\mathrm{Nd}^{+3}$ (vide Fig. 4.5).

A Fig. 4.5 mostra o comportamento da difusividade térmica em função da concentração para a amostra de aluminato de cálcio. Podemos observar que a difusividade varia entre $(5.7-5.2) \times 10^{-3} \mathrm{~cm}^{2} \mathrm{~s}^{-1}$ a medida que a concentração de $\mathrm{Nd}_{2} \mathrm{O}_{3}$ aumenta. 


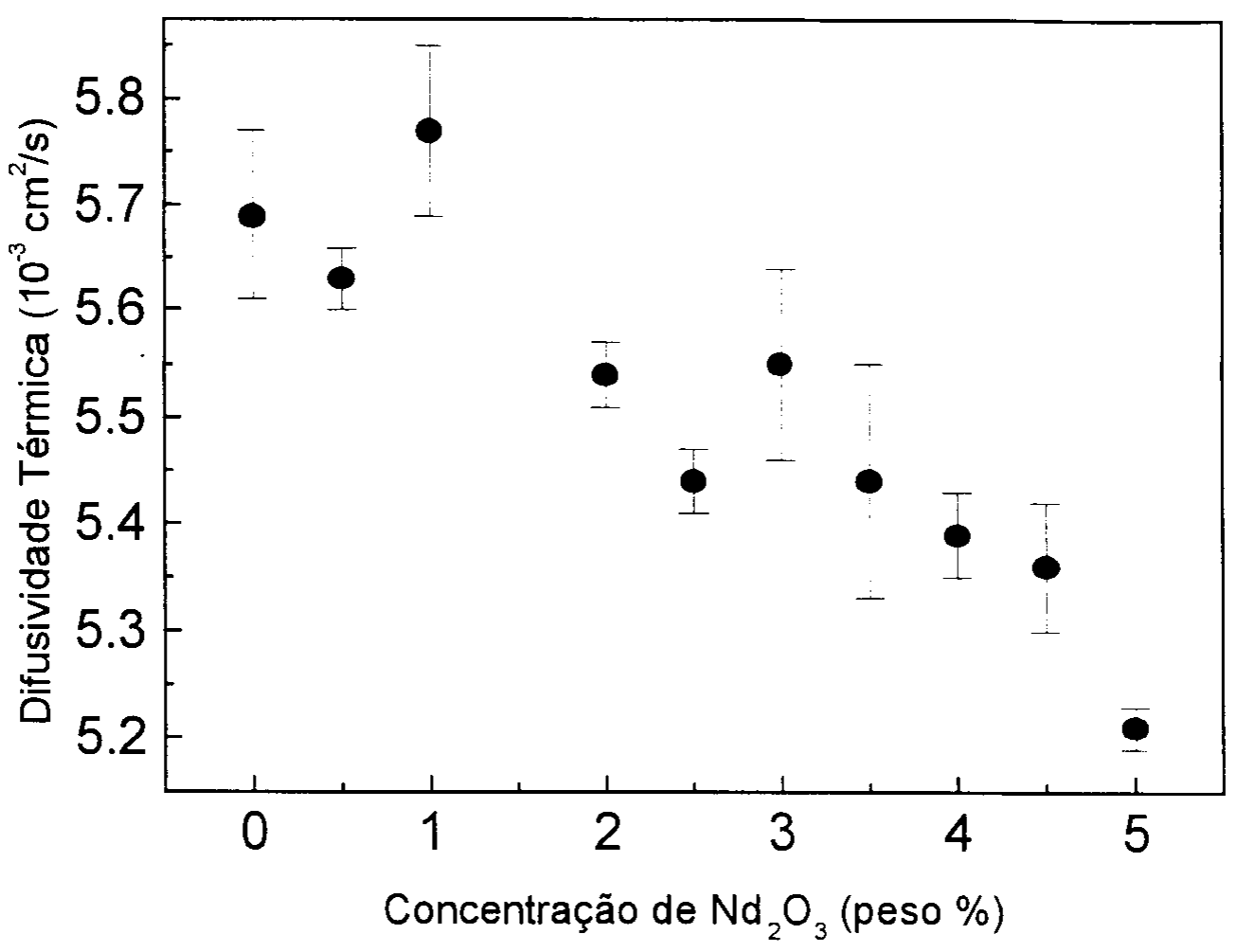

Figura 4.5- Difusividade térmica para várias concentrações de $\mathrm{Nd}_{2} \mathrm{O}_{3}$ em vidros aluminato de cálcio com pouca sílica.

Estas medidas foram realizadas por J. Sampaio, A. A. Andrade, T. Catunda e M. L. Baesso.

\subsection{Medidas de Z-scan}

\subsubsection{Montagem Experimental}

Na Fig. 4.6 temos o aparato experimental que utilizamos para realizar nossas medidas de varredura $\mathbf{z}$. 


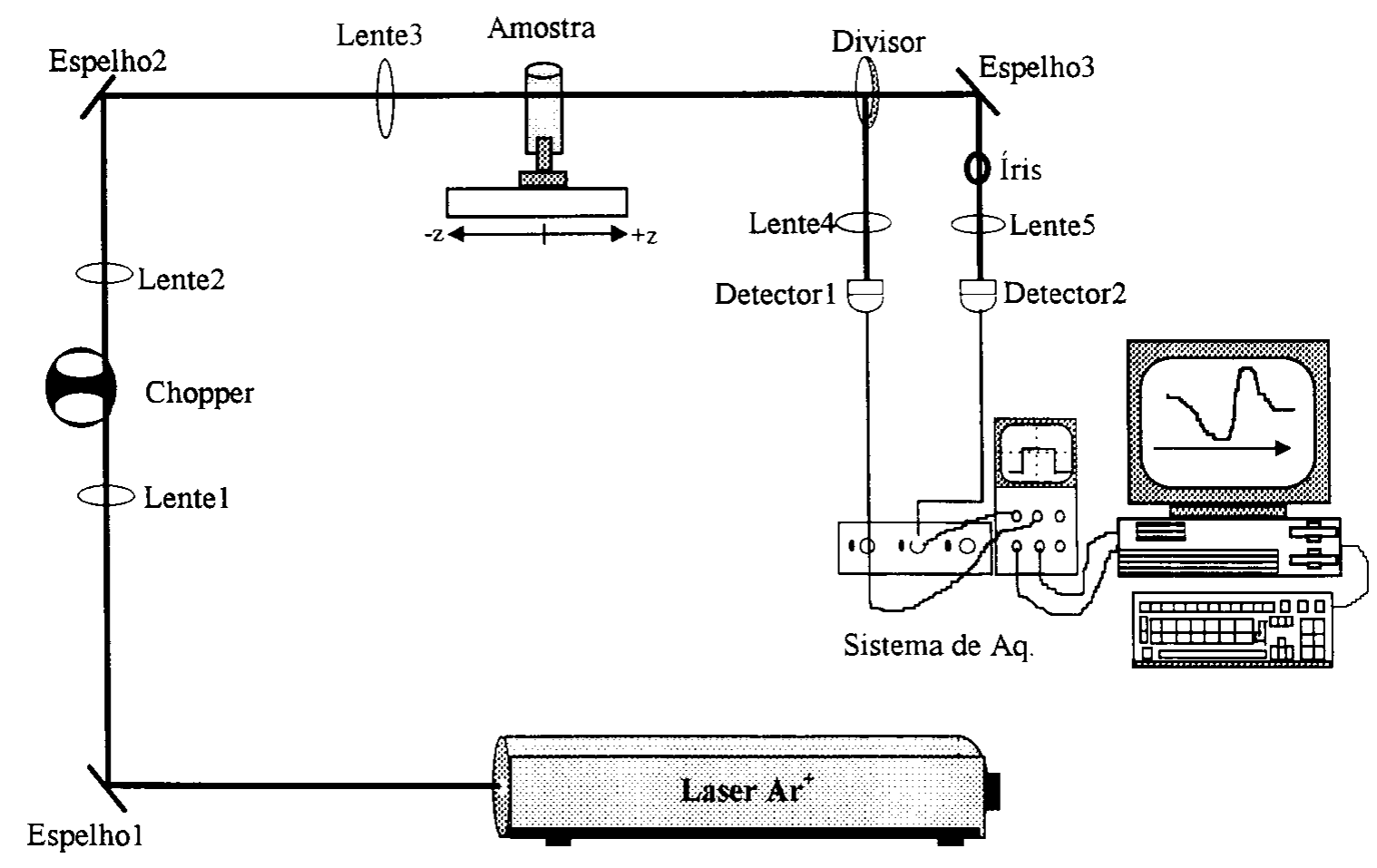

Figura 4.6- Aparato experimental utilizado para as medidas de varredura $\mathrm{z}$. Onde as lentes 1,2 e 3 , tem distâncias focais $f=10 \mathrm{~cm}$ e as lentes 4 e 5 tem $f=4 \mathrm{~cm}$.

$\mathrm{Na}$ montagem exibida acima, foi utilizado como fonte um laser de $\mathrm{Ar}^{+}$(modelo Coherent innova $300 \mathrm{C}$ de $8 \mathrm{~W}$ ). A lente 1 focaliza o feixe na pá do chopper que modula mecanicamente o feixe incidente tal que a transição aberto-fechado é minimizado. A lente 2 recolima o feixe enquanto que a lente 3 produz um foco em $\mathrm{z}=0$. As lentes 4 e 5 tem a finalidade de focalizar o feixe nos detectores. O divisor de feixe gera dois feixes: o feixe transmitido através da iris produz um sinal de varredura z com contribuições das partes real e imaginária do índice de refração não-linear, que é medido pelo detector 2 . O outro feixe, medido pelo detector 1 produz um sinal que apresenta apenas a contribuição da parte imaginária. 
Os efeitos lineares parasitas provenientes do mau polimento ou não paralelismo das faces da amostra, podem ser eliminados pela divisão de uma medida realizada com alta intensidade por outra realizada com baixa intensidade. Imediatamente após a abertura da pá do chopper, em $t_{i}=0$, os estados excitados metaestáveis com tempos de vida longos ( ms) ainda não foram populados (no caso de Lente de População) e/ou a amostra não recebeu energia suficiente para aumentar sua temperatura (no caso de Lente Térmica). Quando o tempo evolui, os estados excitados são populados e/ou a amostra se aquece o suficiente para que os efeitos não-lineares e/ou térmico comecem a ser manifestar. Para $t_{f} \gg \tau$ a população do estado excitado atinge seu estado estacionário e a transmitância apresenta contribuições linear e não-linear. O sistema de aquisição sincronizado com o sinal do chopper, realiza uma medida de transmissão $T\left(t_{i}\right)$ em $t=t_{i}$ e uma segunda $T\left(t_{f}\right)$ em $\mathrm{t}=\mathrm{t}_{\mathrm{f}}$ como mostra a Fig. 4.7. Como imediatamente após a abertura do chopper, em um tempo inicial $\mathrm{t}=\mathrm{t}_{\mathrm{i}} \sim 0$ o laser não induz variação no indice de refração, o sinal pode ser normalizado pela medida em $t_{i}$ pela medida em $t_{f}$. Este método permite também o estudo da evolução temporal do índice de refração o que é muito importante para distinguir efeitos de Lente Térmica (LT) de efeitos Lente População (LP).

Assumindo que o sinal de Lente de População tem um comportamento exponencial descrito pela Eq.(2.6), o efeito de $t_{i}$ e $t_{f}$ poderá ser considerado dividindo o sinal medido por um fator de correção válida para $t_{\mathrm{p}}>>\tau$ e $\mathrm{t}_{\mathrm{P}}>>\tau$ :

$$
\exp \left[-t_{i} / \tau\right]-\exp \left[-t_{f} / \tau\right]
$$


Este fator de correção é importante, visto que uma medida não começa exatamente em $t=0$ como seria esperado(veja Fig. 4.7). $O$ início dá-se em um tempo $t_{i}$, logo após $\mathrm{t}=0$ do sinal, pois é necessário que se leve em consideração o tempo de subida do sinal (tempo que a pá do chopper leva para interromper o feixe laser). O fim da aquisição também ocorre em um tempo $t_{\mathrm{f}}$ (ligeiramente menor do que o final de meio período, T/2, do sinal), veja Apêndice B.

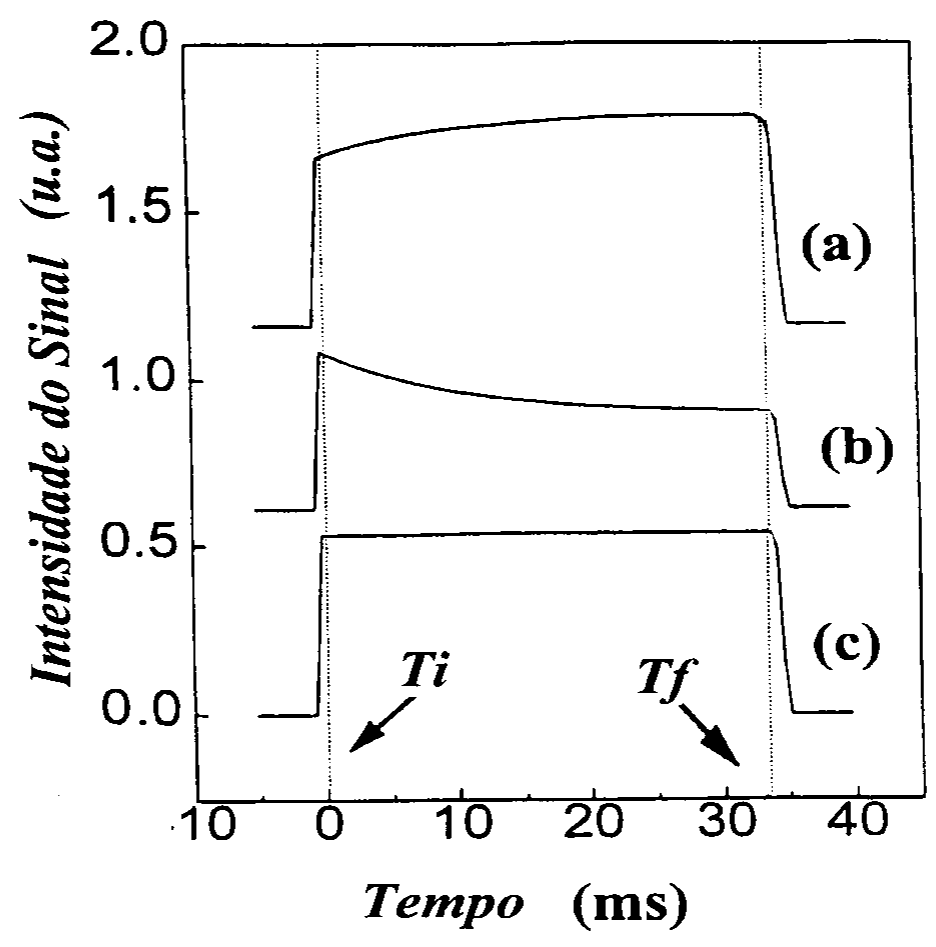

Figura 4.7- Evolução temporal do sinal de Z-scan da amostra de $\mathrm{GdAlO}_{3}: \mathrm{Cr}^{+3}$ $\left(\mathrm{n}_{2}>0\right)$, colocada em (a) $\mathrm{z}=0.85 \mathrm{z}_{0}$, (b) $\mathrm{z}=-0.85 \mathrm{z}_{0}$ e (c) $\mathrm{z}=7 \mathrm{z}_{0}$ [10].

\subsubsection{Resultados em cristais dopados com $\mathrm{Cr}^{+3}$}

Fizemos medidas em dois cristais dopados com $\mathrm{Cr}^{+3}, \mathrm{SrAlF}_{5}: \mathrm{Cr}^{+3}$ e LiSAF, uma vez que estes cristais é de grande interesse da comunidade de lasers.

Nós excitamos o $\mathrm{SrAlF}_{5}: \mathrm{Cr}^{+3}$ com um laser de $\mathrm{Ar}^{+}$em $\lambda_{\mathrm{exc}}=488 \mathrm{~nm} \mathrm{em}$ ressonância com a banda de absorção ${ }^{4} \mathrm{~T}_{1}$ centrada em $430 \mathrm{~nm}$ (vide espectro de 
absorção da Fig.2.2) e com $w_{0}=20 \mu \mathrm{m}$. Todas as medidas foram realizadas com a polarização do laser perpendicular ao eixo c. Nossa amostra tem uma espessura $\mathrm{L}=3.1 \mathrm{~mm}$ e concentração de $\mathrm{Cr}^{+3}$ de 0.8 at. $\%\left(\mathrm{CrF}_{3}\right.$ substituindo $\mathrm{AlF}_{3}$ fusão $)$ o qual corresponde a $\mathrm{N}_{0}=8.9 \times 10^{19} \mathrm{~cm}^{-3}$. Em $488 \mathrm{~nm}$ nós medimos o coeficiente de absorção $\mathrm{A}=1.2 \mathrm{~cm}^{-1}$ e usando o tempo de vida $\tau_{0}=93 \mu \mathrm{s}$ (a temperatura ambiente), através da Eq. 2.5 a nós estimamos $I_{s}=330 \mathrm{~kW} / \mathrm{cm}^{2}$.

As medidas de $Z$-scan foram realizadas em duas diferentes freqüência do chopper, com a finalidade de discriminar os efeitos de LP e LT. A Fig. 4.8 mostra nossos dados de uma medida realizada com freqüência do chopper igual $822 \mathrm{~Hz}$ e com uma íris aberta e outra fechada. A medida foi realizada em um tempo $t_{\mathrm{i}}=20 \mu \mathrm{s}$ e $\mathrm{t}_{\mathrm{f}}=300 \mu \mathrm{s}$. O propósito desta medida é escolher $\tau_{0}<\mathrm{t}_{\mathrm{f}}<\mathrm{t}_{\mathrm{c}}$ de maneira a termos em $\mathrm{t}=\mathrm{t}_{\mathrm{f}}$, uma maior contribuição do sinal de LP do que do sinal de LT. Os dados da medida realizada com a íris aberta $S_{1}=100 \%$, apresenta um decréscimo na transmitância perto do foco devido a absorção do estado excitado. Para medirmos $\mathbf{n}_{2}$, nós dividimos o sinal da íris com abertura $S_{2}=50 \%$ pela medida com $S_{1}=100 \%$, como geralmente é feito na técnica de Z-scan[4]. O resultado da razão da curva indica: $\mathrm{n}_{2}=(6.6+1.7 \mathrm{i}) \times 10^{-11} \mathrm{~cm}^{2} / \mathrm{W}$, um índice de refração não-linear $\mathrm{n}_{2}$ ' positivo e $\mathrm{n}_{2}$ " negativo (comportamento de absorvedor saturável reverso). Com estes resultados pudemos calcular $\Delta \alpha=3.1 \times 10^{-26} \mathrm{~cm}^{3}$ e $\Delta \sigma=-1.7 \times 10^{-20} \mathrm{~cm}^{2}$. A medida foi realizada em regime de baixa intensidade, $\mathrm{I}_{0}=2 \mathrm{P} / \pi \mathrm{w}^{2}$, logo $\mathrm{I}_{0}<<\mathrm{I}_{s}$, tal que os efeitos de saturação pode ser desprezado $[9,10]$. 


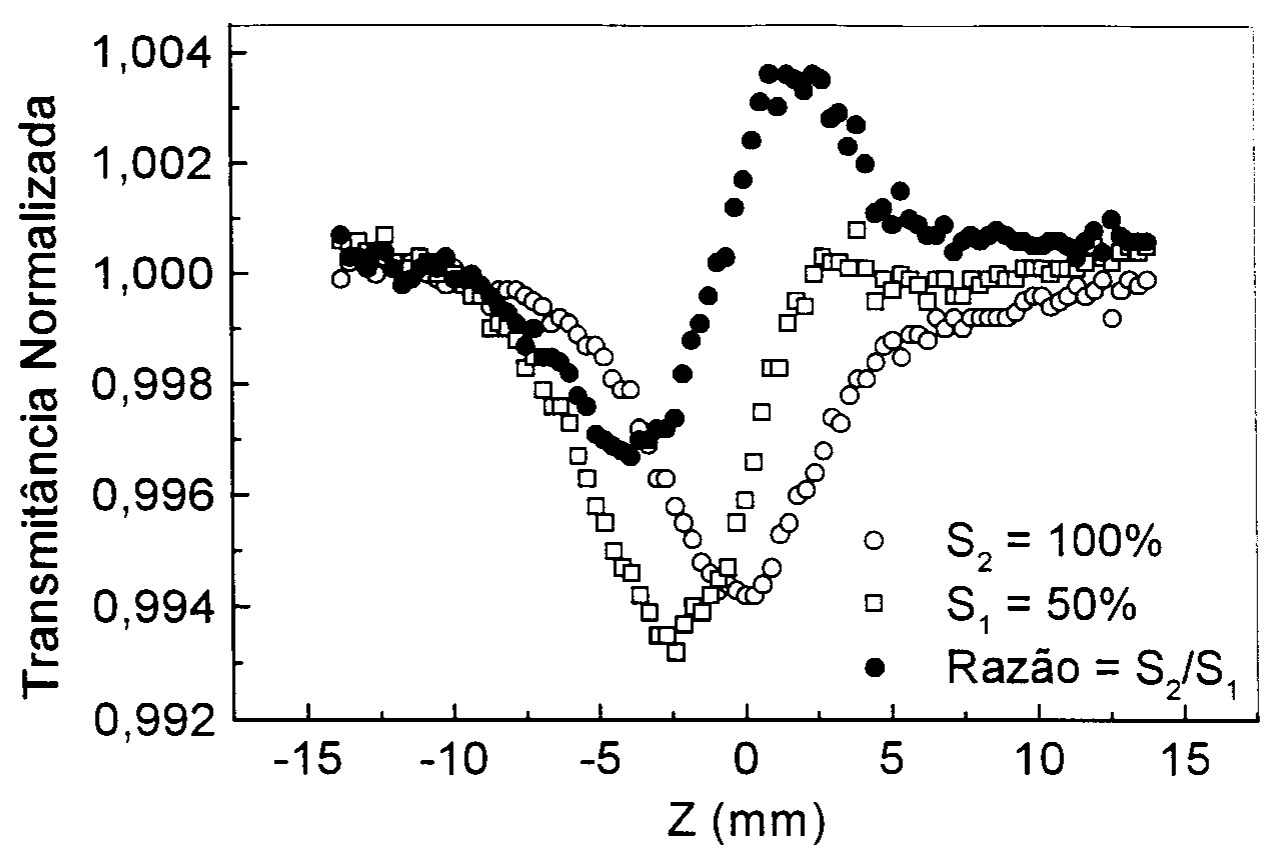

Figura 4.8- Transmitância normalizada obtida na amostra $\mathrm{SrAlF}_{5}: \mathrm{Cr}^{+3}$ com potência do laser $(P=90 \mathrm{~mW})$, frequiência do chopper $(f=822 \mathrm{~Hz})$. Onde $S_{1}=100 \%$ abertura ( $\left.\circ\right), S_{2}=50 \%$ abertura $(\square)$ e razão $S_{2} / S_{1}(\bullet)$.

As medidas em tempo longo, ou seja, baixa freqüência $(f=186 \mathrm{~Hz})$, foram realizadas num intervalo entre $t_{i}=0.46 \mathrm{~ms}$ e $t_{\mathrm{f}} \sim 2.5 \mathrm{~ms}$. Neste caso, a curva com a íris fechada indica uma não-linearidade negativa, a qual é uma característica usual de nãolinearidade térmica em fluoretos que apresentam ds/dT $<0$ [11]. Nós notamos que como $t_{i}>>t_{0}$, em $t=t_{i}$ o sinal LP está próximo do valor estacionário $\left(\right.$ pois $t_{i}>>\tau_{0}$ ) tal que ele não contribui para a razão. 


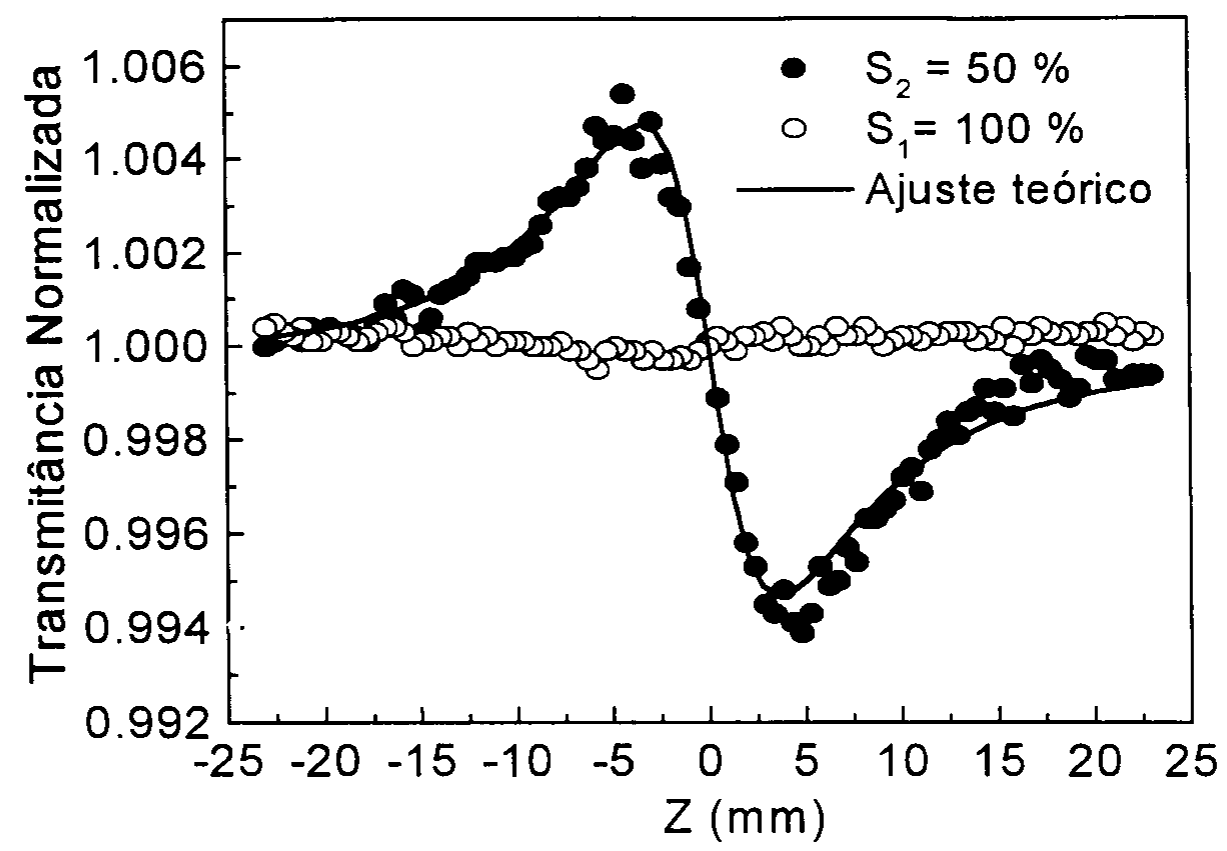

Figura 4.9- Transmitância normalizada obtida na amostra $\mathrm{SrAlF}_{5}: \mathrm{Cr}^{+3}$ com potência do laser de $P=175 \mathrm{~mW}$ e freqüencia do chopper $\mathrm{f}=186 \mathrm{~Hz}$, onde $S_{1}$ $=100 \%(\mathrm{O})$ e $\mathrm{S}_{2}=50 \%(\bullet)$. O ajuste teórico com a expressão de LT forneceu $\mathrm{z}_{0}=2.6 \mathrm{~mm}$.

Podemos observar nesta figura que o valor entre o pico e o vale é $\Delta Z_{p v}-3.4 z_{0}$, como é esperado no caso de não-linearidade térmica, (veja Eq.3.42 para o caso $\mathrm{m}=1$ ) [20, 21]. Esta é uma importante diferença entre o efeito de LT e o efeito de LP. Em um meio tipo Kerr, onde o índice de refração é dado por $n=n_{0}+n_{2} I$, se o perfil de intensidade do laser I(r) for gaussiano, o perfil do índice de refração terá a mesma variação. Usando este fato, pode-se mostrar que para um meio tipo Kerr a diferença entre o pico e o vale é $\Delta Z_{\mathrm{pv}} \sim 1.7 \mathrm{Z}_{0}$, vide Eq. (3.28) do capítulo 3[4]. Porém no efeito de LT, devido a difusão radial de calor, o perfil do índice de refração n(r) é mais largo do que o perfil de intensidade $I(r)$ e por isso causa $u m \Delta Z_{p v}$ maior. Com o uso da expressão de lente térmica com um único feixe $(\mathrm{m}=1)$, ajustamos a curva a qual nos forneceu um $\mathrm{z}_{0}=2.5 \mathrm{~mm}$, o qual está em bom acordo com o $\mathrm{z}_{0}$ obtido através da 
razão da Fig.4.8. O valor de D e $\theta / P$ estão em bom acordo com o valor obtido com a técnica de Lente Térmica (Fig. 4.4).

Para o LiSAF com concentração de 7 at $\%$ de $\mathrm{Cr}^{+3}$, calculamos $\mathrm{N}_{0}=6.2 \times 10^{20}$ íons $\mathrm{cm}^{-3}$. Nós encontramos o coeficiente de absorção $\mathrm{A}=3 \mathrm{~cm}^{-1}$ e usando o tempo de vida $\tau_{0}=67 \mu \mathrm{s}[22]$, nós estimamos $I_{s}=621 \mathrm{~kW} / \mathrm{cm}^{2}$. Nossas medidas foram realizadas com freqüência $(f=822 \mathrm{~Hz})$ e novamente para medirmos $n_{2}$, dividimos o sinal da 'ris com abertura $\mathrm{S}_{2}=50 \%$ pela medida com $\mathrm{S}_{1}=100 \%$. O resultado dessas medidas nos forneceu $\mathfrak{n}_{2}=(0.33+3.9 i) \times 10^{-11} \mathrm{~cm}^{2} / \mathrm{W}$. Com estes resultados calculamos $\Delta \alpha=8.4 \times 10^{-26} \mathrm{~cm}^{3}$ e $\Delta \sigma=2 \times 10^{-20} \mathrm{~cm}^{2}$.

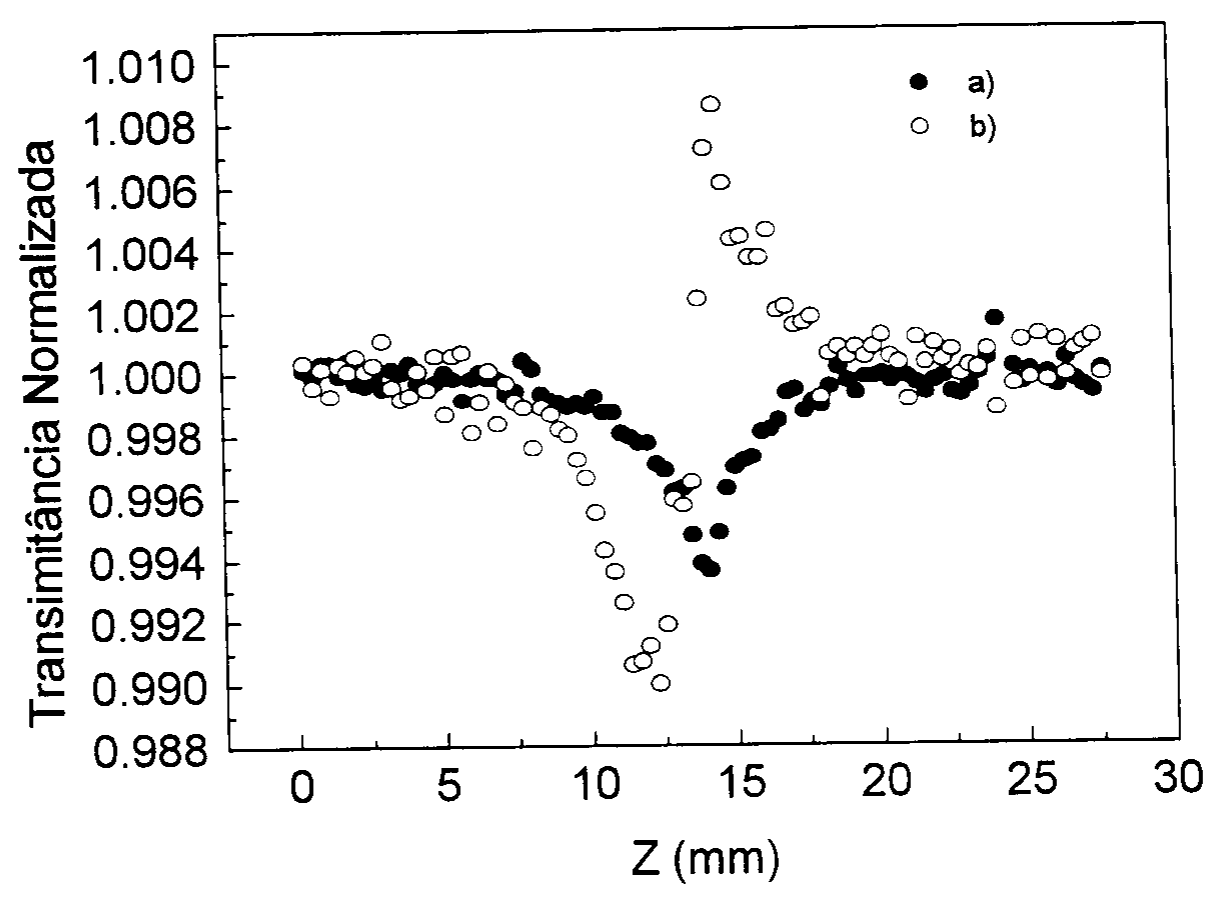

Figura 4.10- Transmitância normalizada obtida no LiSAF com potência do laser de $P=95 \mathrm{~mW}$ ) e freqüência do chopper $\mathrm{f}=822 \mathrm{~Hz}$. A curva a) é o resultado da medida feita com $\mathrm{S}_{1}=100 \%$ e a curva b) é o resultado da divisão da medida feita com $S_{2}=50 \%$ por $S_{1}=100 \%$. 


\subsubsection{Efeitos de Saturação}

A ação laser em cristais fluoretos dopados com $\mathrm{Cr}^{+3}$ é limitada por diversos que aquecem o cristal. Isto foi demonstrado em diversos experimentos, por exemplo de ação laser [23] e medidas de ganho em LiSAF [24]. Como em todo meio laser, a diferença de energia entre o fóton absorvido e o emitido é convertida em calor causando o processo conhecido como "quenching" térmico da fluorescência. Além disso efeitos de transferência de energia ("up-convertion") entre pares de $\mathrm{Cr}$ que causam decaimento não-radiativo[24]. Estes processos fazem com que a potência emitida e o ganho do laser atingem um máximo com potências de excitação muito menores que as esperadas considerando-se o efeito de saturação usual.

Em nossas medidas variamos a potências do laser de bombeio e observamos que a medida que aumentamos a potência, a variação de fase $\Delta \Phi_{0}$ atinge um valor máximo e depois decresce. A Fig. 4.11 mostra nossos resultados, onde cada ponto do gráfico representa uma varredura $Z$-scan com abertura $S_{2}=50 \%$ normalizada por $S_{1}=$ 100\%. A Fig. 4.12 mostra os dados das medidas de Z-scan com a íris em abertura $S_{1}=100 \%$, com a qual podemos determinar a absorção não linear do cristal. 


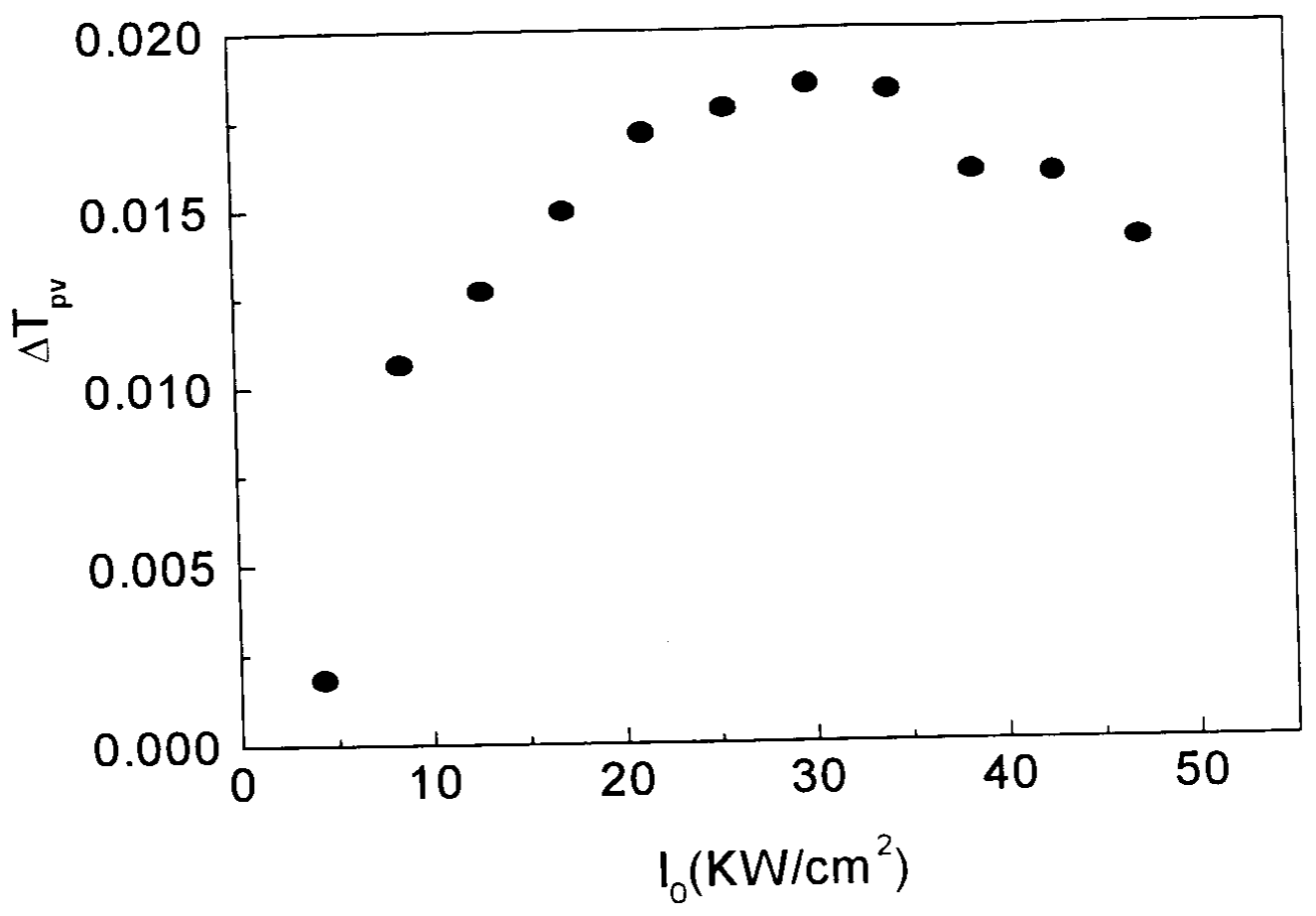

Figura 4.11- Medidas de Z-scan realizadas em várias potências do laser. $\mathrm{O}$ valor de $\Delta \Phi_{0}$ foi obtido através do ajuste de Z-scan realizado na curva $S_{2}=50 \%$ normalizado pela curva $S_{1}=100 \%$.

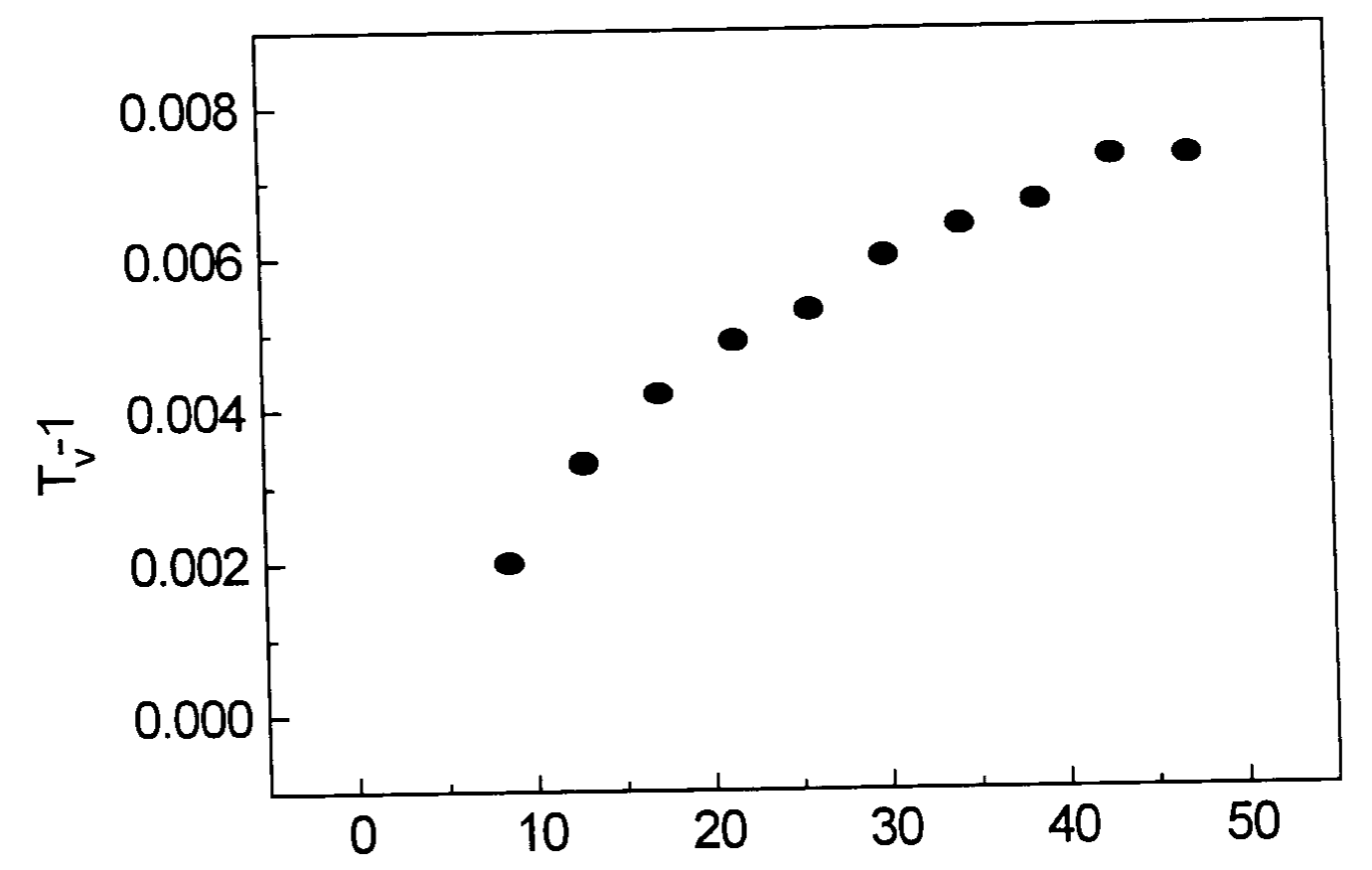

Figura 4.12- Medidas de absorção não-linear por Z-scan com abertura $\mathrm{S}_{1}=100 \%$. 
Obtivemos medidas de transiente, colocando a amostra na posição de vale da razão entre as curvas $S_{2}$ e $S_{1}\left(S_{2} / S_{1}\right.$ - Fig. 4.8). A Fig. 4.13 mostra que o sinal com a íris aberta se comporta similarmente a $\mathrm{N}_{\mathrm{Ex}}(\mathrm{t})$ dado pela Eq.(2.6) como esperado para uma absorção não-linear devido ao efeito de LP. A Fig. 4.14 mostra que a razão $\mathrm{S}_{2} / \mathrm{S}_{1}$ tem um comportamento similar, o qual também caracteriza um sinal de LP para uma refração não-linear. Os dados das curvas $S_{1}=100 \%$ e $S_{2} / S_{1}$ foram obtidos com um ajuste de decaimento exponencial, ond? encontramos $\tau=80 \mu \mathrm{s}$ e $\tau=67 \mu \mathrm{s}$, respectivamente.

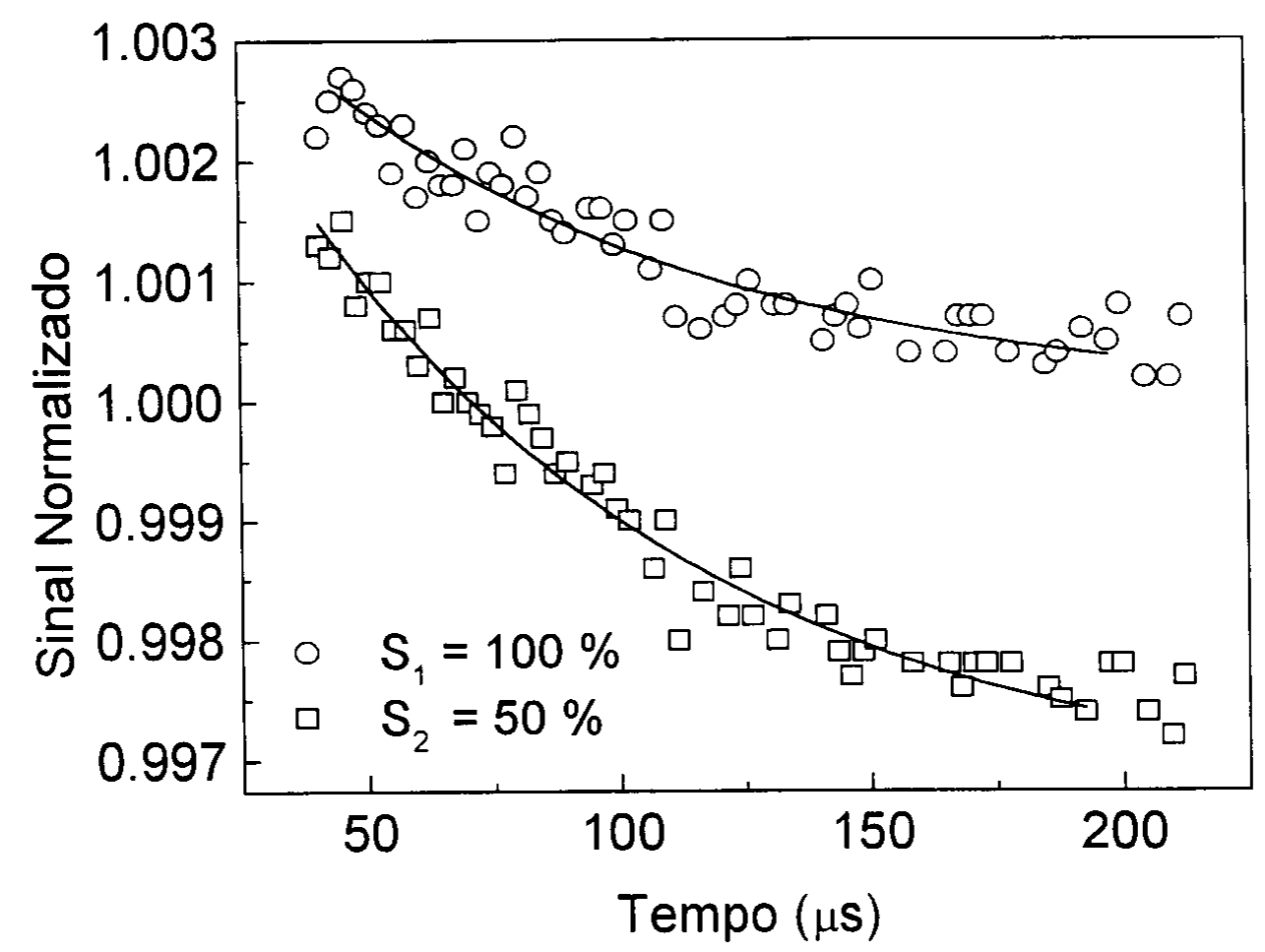

Figura 4.13- Dependência do sinal com a amostra $\mathrm{SrAlF}_{5}: \mathrm{Cr}^{+3}$ na posição de vale $\left(z=-0.85 z_{0}\right)$, com potência do laser $P=335 \mathrm{~mW}$ e freqüência do chopper $f=822 \mathrm{~Hz}$. A parte (a) mostra a medida com a íris aberta $S_{1}=100 \%$ (O) e com a íris em $\mathrm{S}_{2}=50 \%(\square)$. A linha sólida representa um simples decaimento exponencial $\operatorname{com} \tau=80 \mu$ s para $(\mathrm{O})$. 


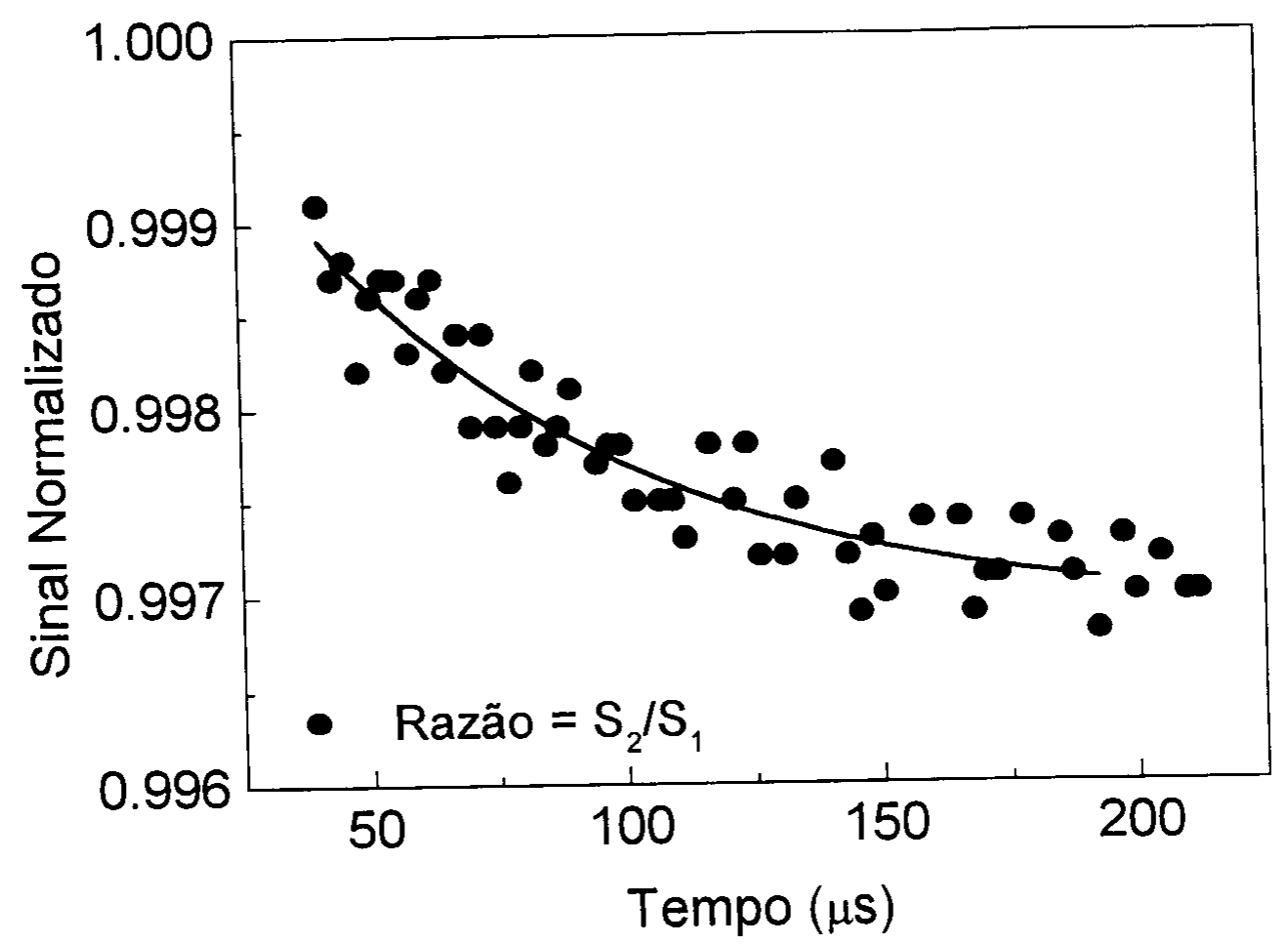

Figura 4.14- Razão das curvas $S_{2} / S_{1}(\bullet)$. O ajuste exponencial forneceu $\tau=67 \mu \mathrm{s}$.

Os tempos de decaimento encontrados, são ligeiramente menores que o tempo de vida $\tau=93 \mu$ s, e esta diferença nós acreditamos que sejam devidos a efeitos de saturação da população do estado excitado. A Eq.(2.7a) mostra que o tempo de resposta de $\mathrm{N}_{E x}$ diminui com o aumento da intensidade e este comportamento já foi observado em mididas de Z-scan na alexandrita[1]. Nesta medida, nós tínhamos $\mathrm{I}_{0}=$ $53 \mathrm{~kW} / \mathrm{cm}^{2} \sim 0.71 \mathrm{I}_{\mathrm{s}}$ e da Eq.(3.7a) encontramos $\tau \sim 80 \mu$ s o qual está em acordo com o resultado da curva $S_{1}$. O pequeno valor do tempo de vida $(\tau)$ obtido com a razão $\mathrm{S}_{2} / \mathrm{S}_{1}$, possivelmente seja devido a uma pequena contribuição do efeito de $\mathrm{LT}$.

Ainda com a amostra na posição de vale, foram realizadas medidas variando a potência do laser (vide Fig. 41.5). 


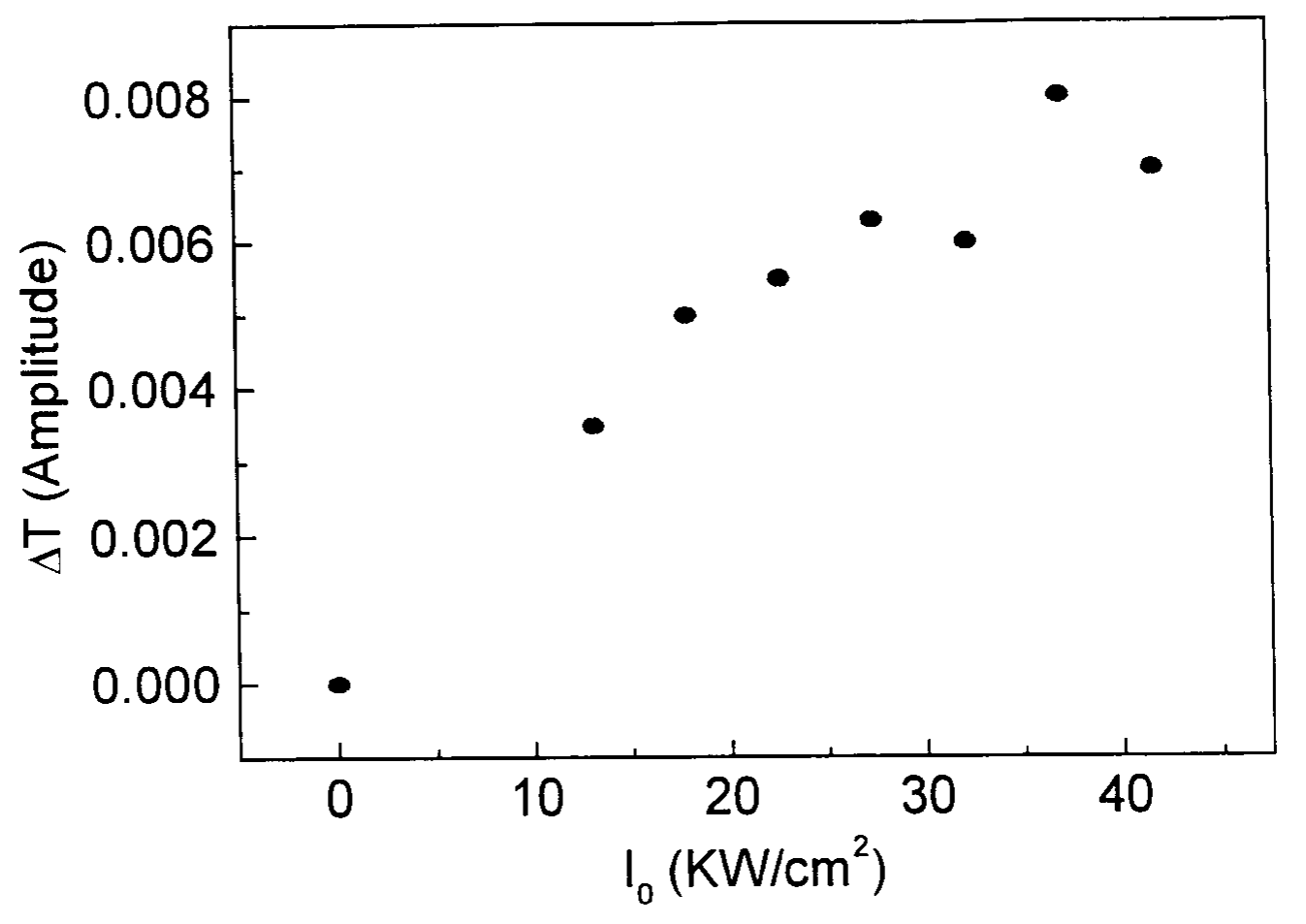

Figura 4.15- Medidas transiente no ponto de vale. Abertura da íris $\mathrm{S}_{1}=100 \%$.

\subsubsection{Resultados em sólidos dopados com $\mathrm{Nd}^{+3}$}

Nos vidros dopados com $\mathrm{Nd}^{+3}$, as medidas foram realizadas em $\lambda=514 \mathrm{~nm}$ onde a absorção é maior que em $488 \mathrm{~nm}$ (veja Fig. 2.1) e com w $=17 \mu \mathrm{m}$.

Novamente nossas medidas foram realizadas em duas diferentes freqüências, afim de discriminar o efeito devido a Lente de População do efeito de Lente Térmica [11]. A Fig. 4.16 mostra as medidas de Z-scan resolvido no tempo no vidro fluorindato dopado com $1 \%$ de $\mathrm{Nd}^{+3}$. Em alta freqüência, os resultados mostram que o efeito devido a Lente de População é maior do que o devido a Lente Térmica. A Fig. 4.16a mostra uma curva característica de Z-scan com não-linearidade positiva (para $\mathrm{f}=840 \mathrm{~Hz}$ ) a qual é atribuída ao efeito de Lente de População. Esta curva foi 
normalizada pela medida realizada com abertura total da iris, Fig. 4.17, afim de eliminar o efeito de absorção do estado excitado.

As medidas realizadas em baixa freqüência nos forneceu uma curva invertida, indicando que $\Delta \mathrm{n}<0$ e consequentemente a variação do caminho óptico ds $/ \mathrm{dT}<0$, veja Fig. 4.16b. A partir da curva da LP (Fig. .416a) determinamos $n_{2}=1.06 \times 10^{-10}$ $\mathrm{cm}^{2} / \mathrm{W}$. Para esta amostra temos $\mathrm{N}_{0}=1.97 \times 10^{20}$ ions $/ \mathrm{cm}^{3}, \tau_{0}=470 \mu \mathrm{s}$ e $\mathrm{I}_{\mathrm{s}}=$ $25 \times 10^{4} \mathrm{~W} / \mathrm{cm}^{2}$ de modo que podemos ca ${ }^{1}$ cular o valor da diferença de polarizabilidade, $\Delta \alpha=1.6 \times 10^{-26} \mathrm{~cm}^{3}$. Vidros fluoretos dopados geralmente apresentam um baixo valor para $\Delta \alpha$ e consequentemente baixo efeito de Lente População. Portanto, mostramos que estes dois efeitos devido a LP e LT podem ser temporalmente distinguidos através da freqüência do chopper.

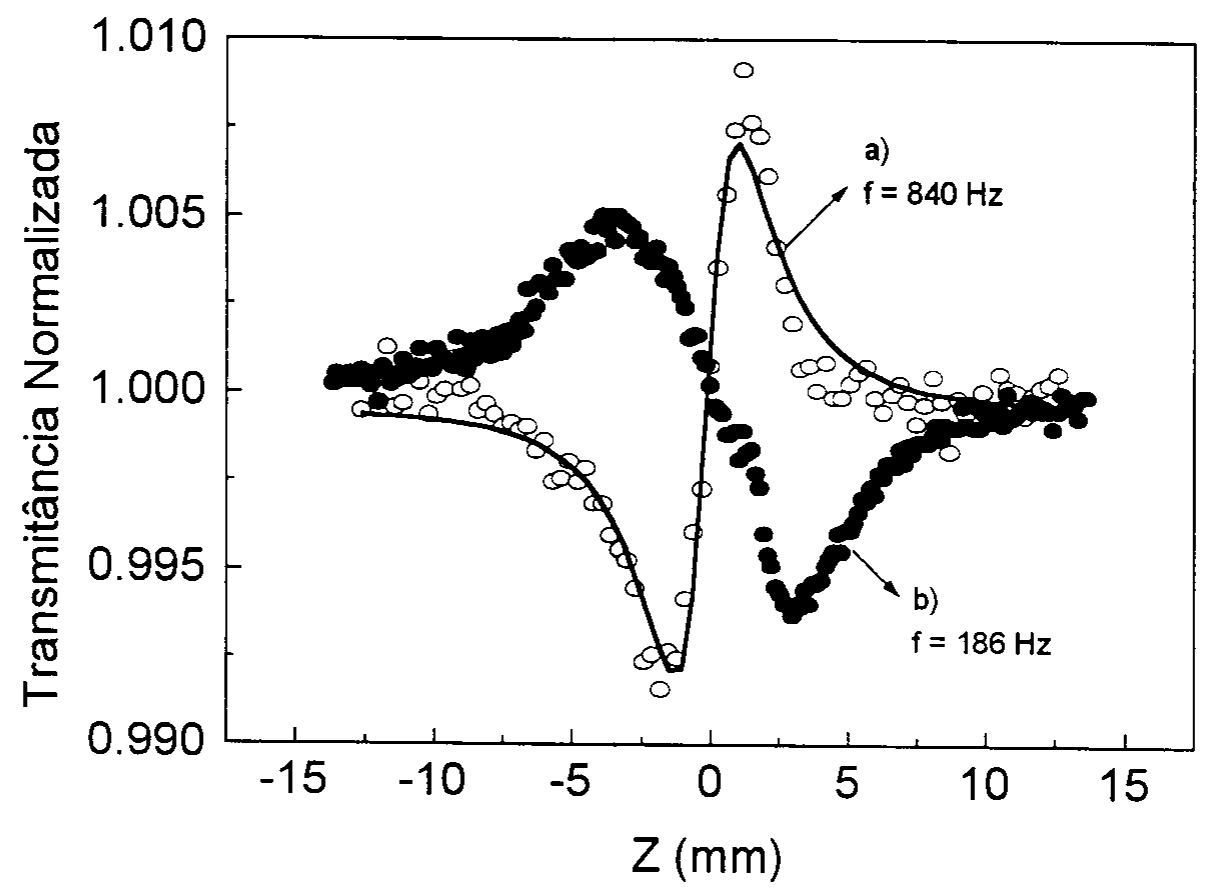

Figura 4.16- Medidas de Z-scan resolvido no tempo do vidro fluorindato dopado com $\mathrm{Nd}^{+3}$, (a) com freqüência $(\mathrm{f}=840 \mathrm{~Hz})$ e potência $(\mathrm{P}=0.187 \mathrm{~W})$ e (b) com freqüência do chopper $(\mathrm{f}=186 \mathrm{~Hz})$ e potência $(\mathrm{P}=0.23 \mathrm{~W})$. 


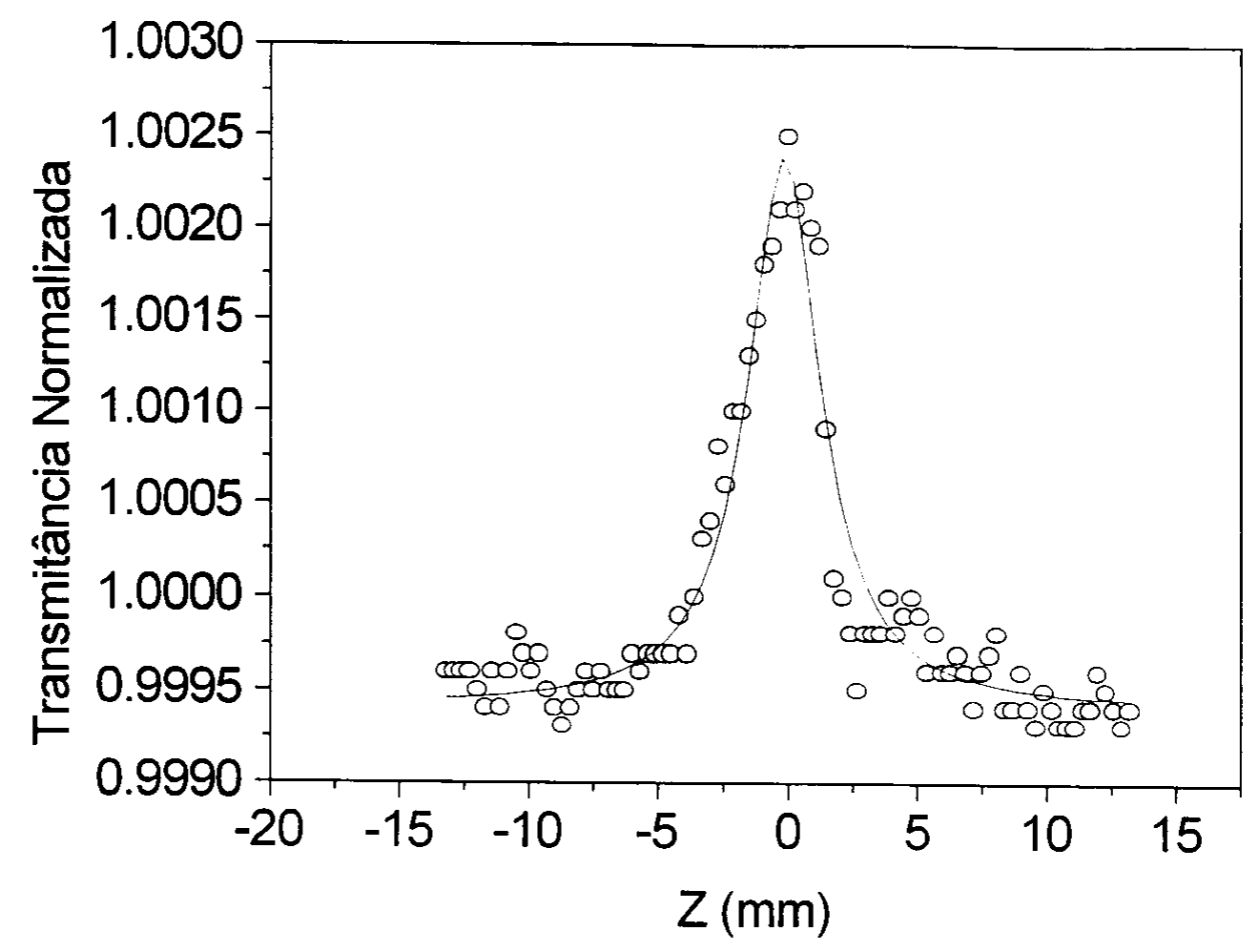

Figura 4.17- Medidas de Z-scan resolvido realizada com abertura $S_{1}=100 \%$, com freqüência $(f=840 \mathrm{~Hz})$ e potência $(\mathrm{P}=0.187 \mathrm{~W})$. A linha cheia é o ajuste teórico.

A curva da Fig. 4.17 foi ajustada por meio da Eq. (3.38), fornecendo $\mathbf{z}_{0}=0.17 \mathrm{~cm}$. Analisando os dados obtidos com a medida realizada com abertura $S_{1}=100 \%$, encontramos $n_{2} "=-5.4 \times 10^{-12} \mathrm{~cm}^{2} / \mathrm{W}$ e consequentemente $\Delta \sigma=-1.7 \times 10^{-21}$ $\mathrm{cm}^{2}$

Realizamos também medidas no ZBLAN com $1 \%$ de $\mathrm{Nd}^{+3}$ e no YAG. Para o ZBLAN (veja Fig. 4.18) em ambas as freqüências do chopper observamos sempre $\Delta \mathrm{n}<0$. Isto indica que ou $\mathrm{n}_{2}<0$ ou o efeito de Lente de População (LP) é muito menor comparado ao efeito de Lente Térmica (LT). Portanto pudemos apenas estimar $\Delta \alpha<0.2 \times 10^{-26} \mathrm{~cm}^{3}$ 


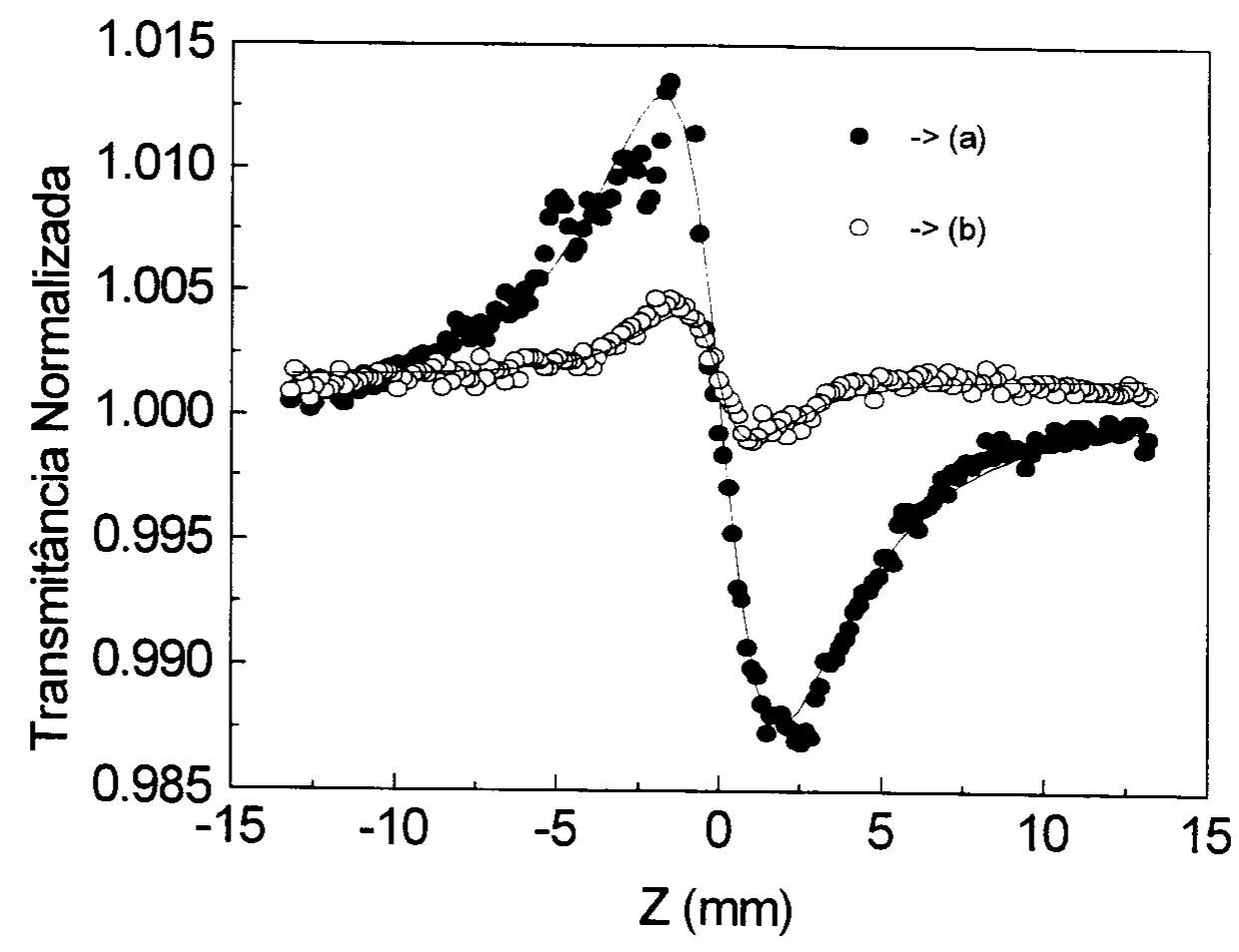

Figura 4.18- Medida realizada no ZBLAN $1 \% \mathrm{Nd}^{+3}$. A curva com círculos fechados, curva (a), representa a medida com freqüência baixa $(f=186 \mathrm{~Hz})$, a curva (b), círculo abertos, representa a medida com freqüência alta $(\mathrm{f}=824 \mathrm{~Hz})$.

No ZBLAN ambas medidas foram realizadas com potência $P=250 \mathrm{~mW}$ e abertura da íris em $S_{2}=50 \%$. A linha cheia na curva (a) é o ajuste teórico feito com a expressão da Lente Térmica para o caso $(m=1)$ e a linha da curva $(b)$ é o ajuste feito com a expressão de Z-scan.

Para o cristal YAG, através de nossas medidas encontramos $n_{2}{ }^{\prime}=1.36 \times 10^{-10}$ $\mathrm{cm}^{2} / \mathrm{W}$ e $\Delta \alpha=4.12 \times 10^{-26} \mathrm{~cm}^{3}$ em bom acordo com[2]. Os resultados estão mostrados nas figuras abaixo. 


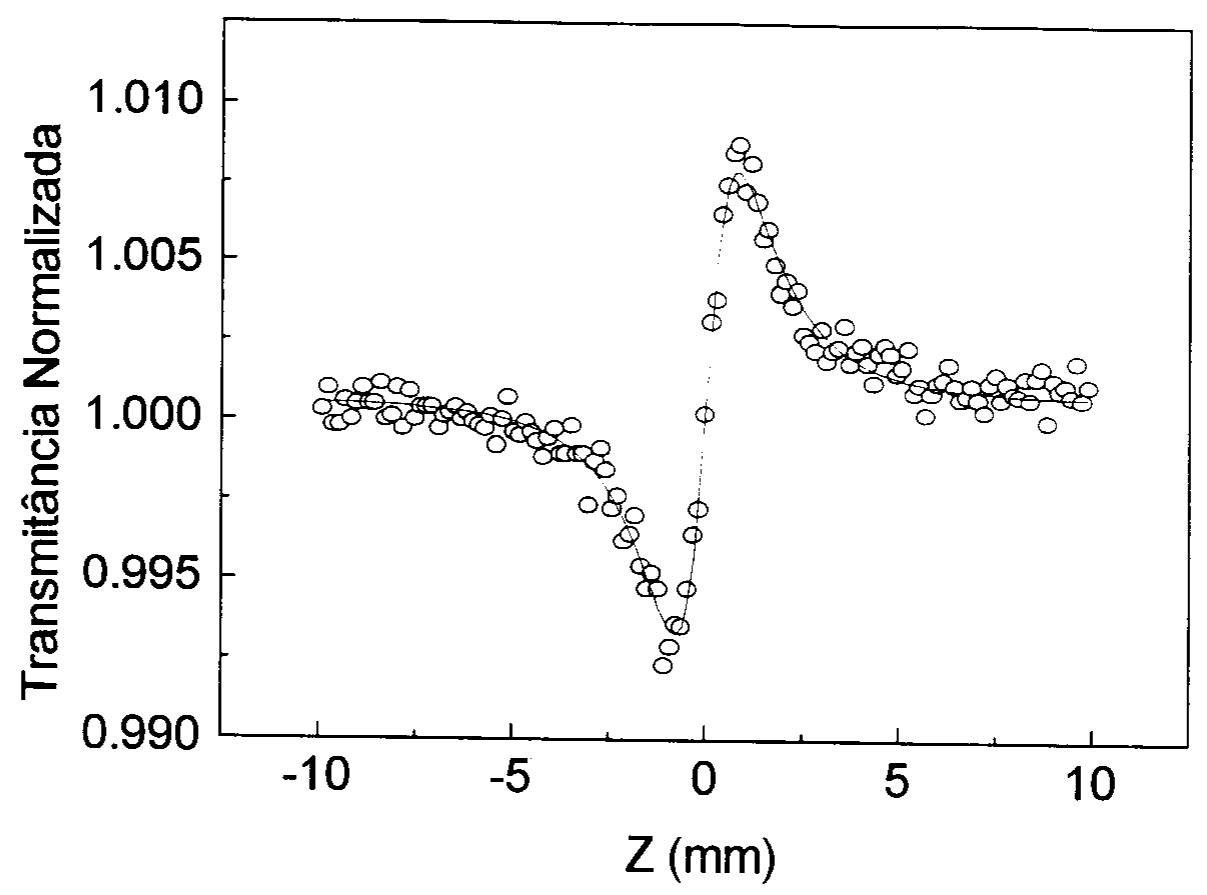

Figura 4.19- Medida de Z-scan no YAG. Abertura da iris $\left(\mathrm{S}_{2}=50 \%\right)$, potência do laser $(P=190 \mathrm{~mW})$ e freqüência do chopper $(\mathrm{f}=824 \mathrm{~Hz})$. Características da amostra: $\mathrm{L}=1.4 \mathrm{~mm}, \tau_{0}=230 \mu \mathrm{s}$ e $\mathrm{I}_{\mathrm{s}}=78.3 \mathrm{KW} / \mathrm{cm}^{2}$

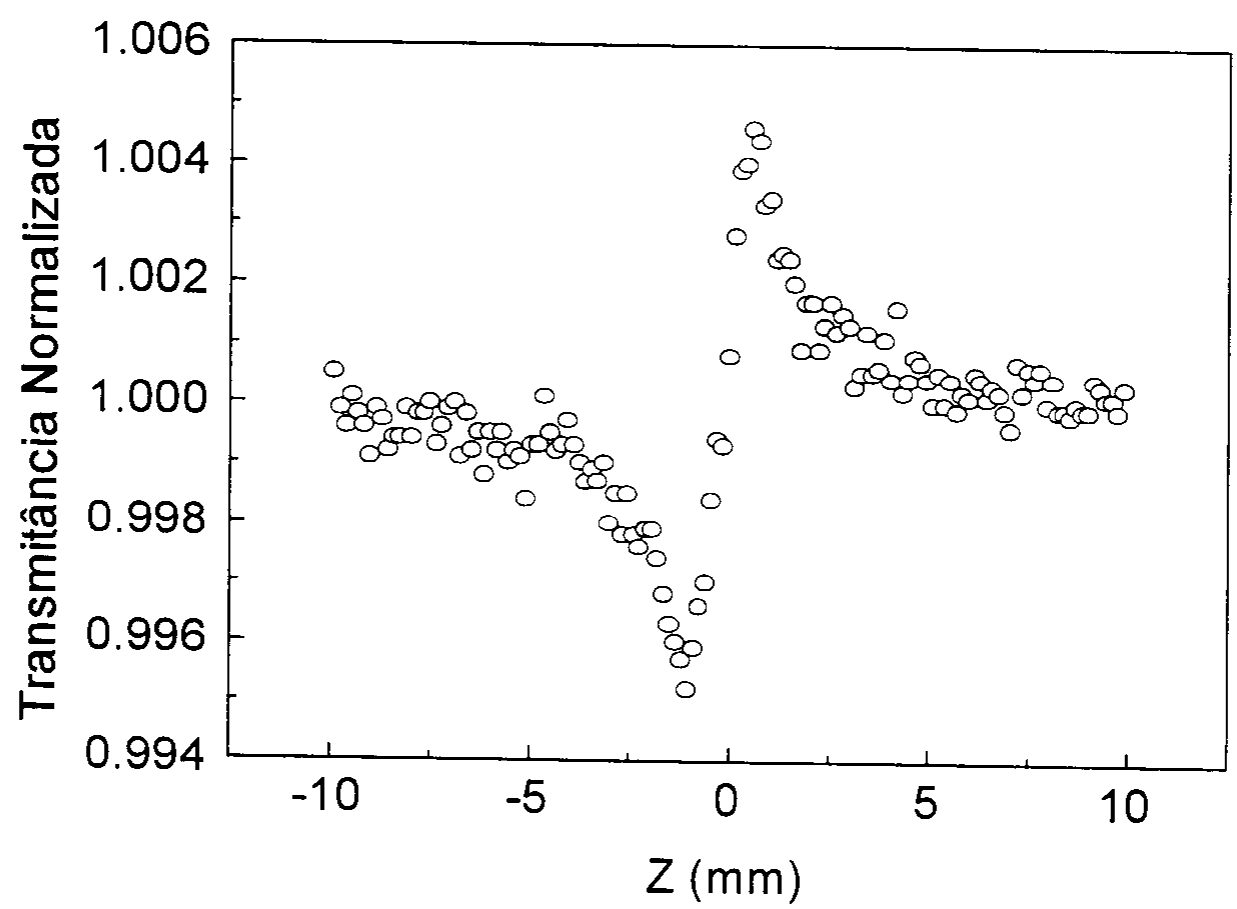

Figura 4.20- Medida de Z-scan no YAG. Abertura da íris ( $\left.\mathrm{S}_{2}=50 \%\right)$, potência do laser $(P=190 \mathrm{~mW})$ e freqüência do chopper $(\mathrm{f}=186 \mathrm{~Hz})$ 


\section{CAPÍTULO 5}

\section{ESTUDO DO EFEITO TÉRMICO E ELETRÔNICO}




\subsection{Efeito térmico e eletrônico simultâneos}

Nos cristais óxidos o efeito de Lente de População é muito maior do que o de Lente Térmica, logo o sinal de Z-scan é atribuído apenas a Lente de População Entretanto, os materiais fluoretos geralmente apresentam diferença de polarizabilidade $\Delta \alpha$ e condutividade térmica $\mathrm{k}$ muito pequenos o que diminui o efeito de Lente de Populaçãc e aumenta o de Lente Térmica. Consequentemente, os efeitos de Lente de População e Lente Térmica podem dar contribuições de mesma magnitude ao sinal de Z-scan e para determinar $\Delta \alpha$ é preciso discriminar estes dois efeitos.

No capítulo anterior mostramos que é possível distinguir temporalmente, os efeitos de Lente de População e Lente Térmica, através da variação da frequêencia do chopper. As Eqs. (3.21) e (3.46) podem ser relacionadas de maneira que podemos determinar qual dos efeitos é mais importante. Primeiro, nós devemos relembrar que em ambos os casos as medidas do sinal é proporcional a potência do laser. Porém, o efeito de LP é proporcional a intensidade do laser, o qual é também proporcional a potência, mas depende da distribuição radial I(r). Portanto, $\Delta \Phi_{0}$ depende criticamente do raio do feixe $w_{0}$, mas $\theta$ depende somente da potência P. Usando as Eqs.(3.21) e (3.46), e relembrando que $\alpha=N_{0} \sigma$, nós obtemos a seguinte expressão:

$$
\frac{\Delta \Phi_{0}}{\theta}=\left(\frac{8 \pi \lambda_{\mathrm{exc}}}{\varphi \mathrm{w}_{0}^{2} \mathrm{ch}}\right)\left(\frac{\mathrm{f}_{\mathrm{L}}^{2} \tau_{0} \Delta \alpha}{\mathrm{n}_{0}}\right)\left(\frac{\mathrm{K}}{\mathrm{ds} / \mathrm{dT}}\right)
$$

onde a fração da energia absorvida e convertida em calor pelo fóton e é dada por $\varphi=$ (1- $\left.\phi \lambda_{\text {exc }} / \lambda_{\text {em }}\right)$. Em sólidos onde há remissão de luz nem toda a energia absorvida é 
transformada em calor. A fração da energia reemitida, que é transformada em calor é proporcional a razão entre as energias do fóton absorvido pela energia do fóton emitido, ou seja, $\phi \lambda_{\text {exc }} / \lambda_{\text {em }}$ onde $\phi$ é a eficiência quântica de emissão, $\lambda_{\text {exc }}$ é o comprimento de onda de excitação e $\lambda_{\mathrm{em}}$ o comprimento de onda de emissão. A Eq.(5.1) é válida para o caso estacionário e no regime de baixa saturação $\left(\mathrm{I}<<\mathrm{I}_{\mathrm{S}}\right)$, onde segundo a Eq. (2.6) $\mathrm{N}_{\mathrm{ex}} \sim \mathrm{N}_{0} \mathrm{I} / \mathrm{I}_{\mathrm{s}}$, e para o caso estacionário ( $\mathrm{t}>>\tau_{0}$ ou $\mathrm{t}_{\mathrm{c}}$ ). Nós também desprezamos a absorção da matriz por usar $A=N_{0} \sigma_{g}$ (onde $N_{0}$ é a concentração do dopante), consequentemente ambos os termos $\Delta \Phi_{0}$ e $\theta$ são proporcionais a $N_{0} \sigma_{\mathrm{g}}$ de tal forma que este termo se cancela na razão $\Delta \Phi_{0} / \theta$. Uma outra consideração deve ser feita nesta equação. Relembrando que $\theta$ definido pela Eq.(3.46) é a diferença de fase do laser de prova induzida pela lente térmica entre $\mathrm{r}=0$ e $\mathrm{r}=1.44 \mathrm{w}_{\mathrm{e}}$. Definimos agora como $\Delta \phi_{\mathrm{th}}$ como sendo o valor da variação de fase estacionária entre $r=0$ e $r \rightarrow \infty$ devido ao efeito de LT, similarmente com a definição de $\Delta \phi_{0}$ dado pela Eq. (3.21). Utilizando as simulações do perfil de temperatura $\Delta T\left(r, t=1000 t_{c}\right)$ obtidas por Shen e Snook $[20,21]$ estimamos que $\Delta \phi_{\mathrm{th}} \sim 3.2 \theta$, portanto a Eq.(5.1) pode ser reescrita como:

$$
\frac{\Delta \phi_{0}}{\Delta \phi_{\tau \mathrm{h}}}=\left(\frac{7.85 \pi \lambda_{\mathrm{exc}}}{\varphi \mathrm{w}_{0}^{2} \mathrm{ch}}\right)\left(\frac{\mathrm{f}_{\mathrm{L}}^{2} \tau_{0} \Delta \alpha}{\mathrm{n}}\right)\left(\frac{\mathrm{K}}{\mathrm{ds}}\right)
$$




\begin{tabular}{|l|c|c|c|c|c|c|}
\hline \multicolumn{1}{|c|}{ Material } & $\begin{array}{c}\mathbf{k} \\
\mathbf{W} \mathbf{c m}^{-1} \mathbf{K}^{-1}\end{array}$ & $\begin{array}{c}\mathbf{D} \\
\mathbf{c m}^{2} \mathbf{s}^{-1}\end{array}$ & $\begin{array}{c}\mathbf{\rho} \\
\mathbf{g c m}^{-3}\end{array}$ & $\begin{array}{c}\mathbf{C} \\
\mathbf{J g}^{-1} \mathbf{K}^{-\mathbf{1}}\end{array}$ & $\mathbf{n}_{\mathbf{0}}$ & $\begin{array}{c}\mathbf{d s} / \mathbf{d T} \\
\mathbf{1 0}^{-6} \mathbf{K}^{-\mathbf{1}}\end{array}$ \\
\hline $\mathrm{Al}_{2} \mathrm{O}_{3}: \mathrm{Cr}^{+3}$ & 0.42 & 0.13 & & & 1.76 & 18.35 \\
\hline${\mathrm{YAG}: \mathrm{Nd}^{+3}}^{+3}$ & 0.13 & 0.046 & 4.56 & 0.59 & 1.82 & 15.3 \\
\hline $\mathrm{YLF}: \mathrm{Nd}^{+3}$ & 0.06 & 0.019 & & & 1.47 & 5.9 \\
\hline $\mathrm{LiCAF}: \mathrm{Cr}^{+3}$ & 0.0458 & 0.0164 & 2.988 & 0.935 & 1.38 & 7.0 \\
\hline $\mathrm{LiSaF}: \mathrm{Cr}^{+3}$ & 0.031 & 0.01 & 3.45 & 0.842 & 1.405 & -7.7 \\
\hline $\mathrm{SrAlF}_{5}: \mathrm{Cr}^{+3}$ & 0.017 & 0.0065 & 3.86 & 0.694 & 1.44 & -1.0 \\
\hline InSBZnGdN:Nd & 0.01 & 4.5 & 4.8 & & 1.5 & -0.67 \\
\hline $\mathrm{ZBLAN}$ & 0.0041 & 0.00155 & 4.45 & 0.6 & 1.48 & -6.1 \\
\hline
\end{tabular}

Tabela 5.1- Parâmetros usados para o cálculo da relação entre as diferenças de efeito de Lente de População e Lente Térmica, $\Delta \Phi_{0} / \Delta \phi_{\mathrm{th}}$ (Eq. 5.2) em diversos materiais. Os dados para o rubi foram retirados de [7]; para o YAG, YLF [25]; para o $\mathrm{LiCaAlF}_{6}: \mathrm{Cr}^{+3}$ de [12] e para o ZBLAN $\operatorname{de}[26]$.

A Eq.(5.2) foi escrita como o produto de três diferentes parêntesis com a finalidade de facilitar sua interpretação. $O$ primeiro termo depende de $\mathrm{w}_{0}$ e da matriz hospedeira devido a $\lambda_{\mathrm{em}}$ e $\varphi$. O segundo parêntesis é proporcional ao efeito de Lente de População e depende fortemente das características da matriz devido a $\tau_{0}$ e $\Delta \alpha$. O terceiro parêntesis é inversamente proporcional ao efeito e Lente Térmica e também depende da matriz hospedeira. Por exemplo, a safira e o YAG apresentam alta condutividade térmica $\left(\mathrm{K}=0.42\right.$ e $0.14 \mathrm{Wcm}^{-1} \mathrm{~K}^{-1}$ respectivamente) enquanto que para o $\mathrm{SrAlF}_{5}$ nós estimamos $\mathrm{K} \sim 0.017 \mathrm{Wcm}^{-1} \mathrm{~K}^{-1}$. Consequentemente, $\Delta \phi_{\text {th }}$ é geralmente maior em fluoretos do que em óxidos devido a sua pequena condutividade térmica $\mathrm{k}$. $\mathrm{O}$ comportamento da variação de fase transiente, também é diferente para 0 efeito de LP e LT. A resposta transiente de LP depende de $\tau_{0}$. Por outro lado, para LT $t_{c}$ depende de $w_{0}$ e D (Eq. 3.48). Usando os dados obtidos neste trabalho 
calculamos $\Delta \Phi_{0} / \Delta \phi_{\text {th }} \sim 0.7$ para nosso experimento de Z-scan no $\mathrm{SrAlF}_{s}: \mathrm{Cr}^{+3}$, onde $\mathrm{w}_{0}=20 \mu \mathrm{m}$. Consequentemente no caso estacionário, as contribuições de LP e LT tem magnitude comparáveis. Entretanto, pudemos separar os dois efeitos por meio de medidas resolvidas no tempo, através da variação da freqüência do chopper. É interessante para observar que um comportamento completamente diferente é esperado para o mesmo íon em matrizes diferentes.

Como exemplo vamos comparar o $\mathrm{SrAlF}_{5}: \mathrm{Cr}^{+3}$ com o rubi $\left(\mathrm{Al}_{2} \mathrm{O}_{3}: \mathrm{Cr}^{+3}\right)$. A safira $\left(\mathrm{Al}_{2} \mathrm{O}_{3}\right)$ apresenta difusividade 20 vezes maior do que o $\mathrm{SrAlF}_{5}$. O valor de $\Delta \alpha$ para o $\mathrm{Cr}^{+3}$ na safira é 5.5 vezes maior que no $\mathrm{SrAlF}_{5}$. Além disso, no rubi $\tau_{0} \sim 3.8 \mathrm{~ms}$ (41 vezes maior do que o tempo do $\mathrm{SrAlF}_{5}: \mathrm{Cr}^{+3}$ ) tal que no rubi o efeito de LT é muito mais rápido no que o LP.

Calculamos ainda a razão de $\Delta \Phi_{0} / \Delta \phi_{\text {th }}$ para outros sólidos os quais estão listados na Tabela 5.2 .

\begin{tabular}{|l|c|c|c|c|c|c|}
\hline \multicolumn{1}{|c|}{ Material } & $\begin{array}{c}\mathbf{1 0}^{-5} \mathbf{F}_{\text {th }} \\
(\mathbf{c m} / \mathbf{W})\end{array}$ & $\begin{array}{c}\mathbf{1 0}^{-30} \mathbf{F}_{\mathbf{0}} \\
\left(\mathbf{s e c . c m}^{-3}\right)\end{array}$ & $\begin{array}{c}\mathbf{t}_{\mathbf{c}} \\
(\mu \mathbf{s})\end{array}$ & $\begin{array}{c}\tau_{\mathbf{0}} \\
(\mu \mathbf{s})\end{array}$ & $\begin{array}{c}10^{-25} \Delta \alpha \\
\left(\mathbf{c m}^{3}\right)\end{array}$ & $\Delta \Phi_{\mathbf{0}} / \Delta \phi_{\text {th }}$ \\
\hline $\mathrm{Al}_{2} \mathrm{O}_{3}: \mathrm{Cr}^{+3}$ & 4.0 & 1100 & 7.7 & 3800 & 1.70 & 995 \\
\hline $\mathrm{YAG}: \mathrm{Nd}^{+3}$ & -9.8 & 8.8 & 53 & 480 & 0.14 & 3.8 \\
\hline $\mathrm{YLF}: \mathrm{Nd}^{+3}$ & 6.26 & 9.2 & 222 & 430 & 0.16 & 6.2 \\
\hline $\mathrm{InSBZnGdN:N^{+3 }}$ & -6.2 & 4.3 & 61 & 175 & $<0.2$ & $<2.5$ \\
\hline $\mathrm{LiCaAlF}_{6}: \mathrm{Cr}^{+3}$ & -24.8 & 7.05 & 92 & 67 & 0.84 & 1.03 \\
\hline $\mathrm{LiSaF}: \mathrm{Cr}^{+3}$ & -150 & $<1.38$ & 333 & 523 & $<0.02$ & $<0.03$ \\
\hline $\mathrm{ZBLAN}$ & 2.37 & 3.7 & 154 & 93 & 0.31 & 0.7 \\
\hline SrAlF $: \mathrm{Cr}^{+3}$ & & & & 214 & 0.46 & 8.3 \\
\hline
\end{tabular}

Tabela 5.2- Parâmetros usados para o cálculo da relação entre as diferenças de efeito de LP e LT, $\Delta \Phi_{0} / \Delta \phi_{\mathrm{th}}$ (Eq. 5.2) em diversos materiais. $\mathrm{F}_{\mathrm{th}}=\mathrm{K}^{-1}$ $\mathrm{ds} / \mathrm{dT}$ é um fator proporcional a $\Delta \phi_{\mathrm{th}}$ e $\mathrm{F}_{0}=\tau_{0} \Delta \alpha \mathrm{f}_{\mathrm{L}}{ }^{2} / \mathrm{n}$ é um fator proporcional a LP. Para estes cálculo foi utilizado $\mathrm{w}_{0}=20 \mu \mathrm{m}$. Os dados para o rubi foram retirados de [7]; para o YAG e YLF [25]; para o $\mathrm{LiCaAlF}_{6}: \mathrm{Cr}^{+3}$ de [12] e para o ZBLAN de [26]. 
Temos portanto dois fatores principais que contribuem para que a razão $\Delta \Phi_{0} / \Delta \phi_{\mathrm{th}}$ seja menor nos fluoretos do que nos óxidos[27]:

$1^{0}-\Delta \alpha$ do $\mathrm{Cr}^{+3}$ ou do $\mathrm{Nd}^{+3}$ é menor em fluoretos do que em óxidos;

$2^{0}$ - A condutividade térmica é bem menor nos fluoretos causando maior efeito térmico do que nos óxidos.

\subsection{Simulações Teóricas}

A superposição dos efeitos de LT e LP, é um problema geral da técnica de Zscan, não só no caso de sólidos mas também em líquidos e [28-30], polímeros[31], etc. Mostramos no capítulo 4, que em alguns casos conseguimos separar a contribuição destes dois efeitos, no $\mathrm{SrAlF}_{5}: \mathrm{Cr}^{+3}[6]$ e no $\operatorname{InSbZn}: \mathrm{Nd}^{+3}[5]$.

Com o intuito de verificar até que ponto conseguimos distinguir temporalmente a contribuição dos efeitos, Lente Térmica e não-linear, elaboramos um modelo teórico que considera a ação simultânea destes efeitos. A expressão obtida foi (veja apêndice C):

$$
\Delta T=\frac{4 \Delta \Phi_{0} x(1-\exp [-t / \tau])}{\left(1+x^{2}\right)\left(9+x^{2}\right)}+\theta \tan ^{-1}\left(\frac{2 x}{\left(9+x^{2}\right) \frac{t_{c}}{2 t}+3+x^{2}}\right)
$$

onde $\Delta \Phi_{0}$ é a diferença de fase da LP dada pela Eq. 3.21, $\mathrm{x}=\mathrm{z} / \mathbf{z}_{0}$, onde $\mathrm{z}$ é a posição da amostra normalizada pela distância confocal $z_{0}(z=0$ quando a amostra está no foco), $\theta$ a diferença de fase devido ao efeito de LT, $t$ o tempo de medida e $t_{c}$ o tempo 
de resposta térmico característico da amostra (vide Eq. 3.46). O primeiro termo representa a equação que rege o efeito não-linear de LP (equação para o Z-scan) [4] e o segundo o efeito térmico (equação de LT com $m=1$ tal como definida em [32]).

A Fig. 5.1 mostra curvas de Z-scan calculadas através da Eq. 5.3. Neste exemplo mostramos simulações de um experimento com o $\mathrm{Nd}: \mathrm{YAG}$, onde a contribuição do efeito térmico no tempo curto $(300 \mu$ s) é praticamente desprezível, in licando desta forma que podemos separar temporalmente a contribuição dos dois efeitos.

Na Fig. 5.2 mostramos a curva de Z-scan e de Lente Térmica. Podemos observar que, no caso da lente térmica ocorre um alargamento da curva em relação àquela devido ao efeito eletrônico. Ou seja, há um aumento na distância $\Delta \mathrm{Z}_{\mathrm{pv}}$. Para o efeito eletrônico esta distância é de $1.7 \mathrm{z}_{0}$, enquanto que para o efeito térmico $\Delta Z_{p v} \sim 3.4 z_{0}$. Na Fig. 5.3 temos a simulação do efeito de LT na técnica de Z-scan para vários valores de $t / t_{c}$. Podemos verificar que para $t / t_{c} \geq 5$ o perfil de temperatura $\Delta \mathrm{T}(\mathrm{r})$ praticamente atinge o estado estacionário. 


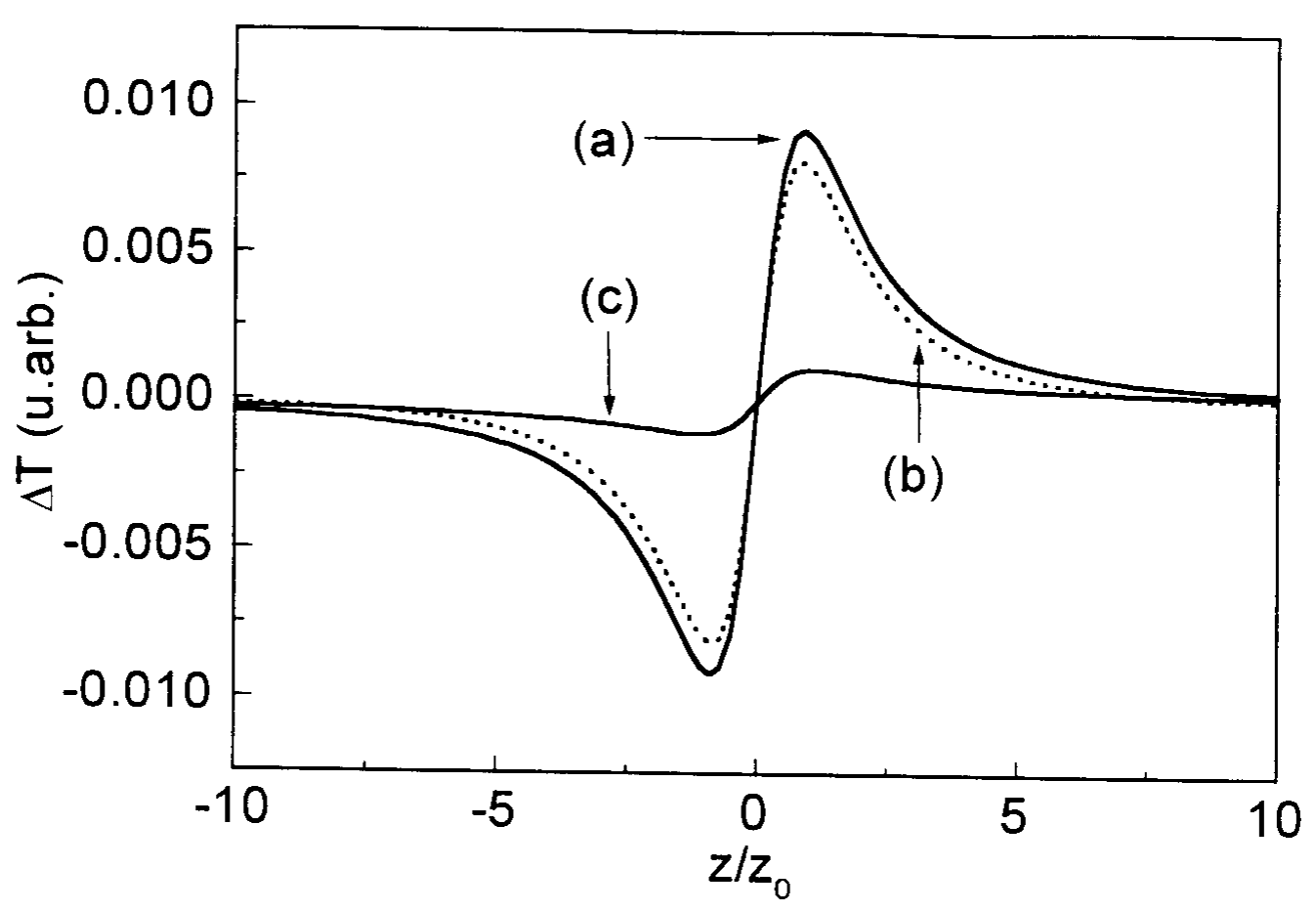

Figura 5.1 - Simulações teóricas para o YAG, com $\Delta \Phi_{0}=0.06, \theta=0.013$, $\mathrm{t}_{\mathrm{c}}=12 \mu \mathrm{s}$ e $\mathrm{t}=350 \mu \mathrm{s}$. A curva (a) representa a simulação onde é levado em conta os dois efeitos. A curva (b) contribuição do efeito de $\operatorname{LP}(\theta=0)$ e a curva (c) o efeito de LT $\left(\Delta \Phi_{0}=0\right)$.

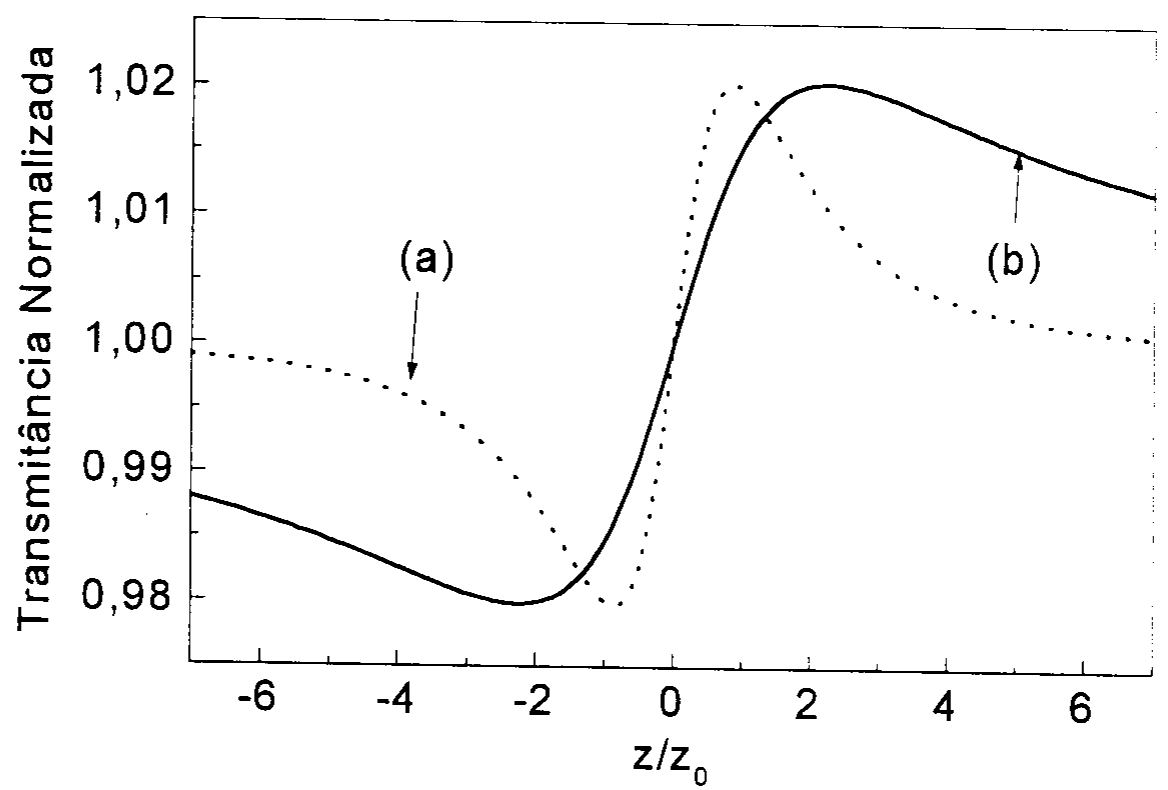

Figura 5.2 - Simulações teórica. Em (a) temos a curva teórica de Z-scan e (b) a curva de Lente Térmica. 


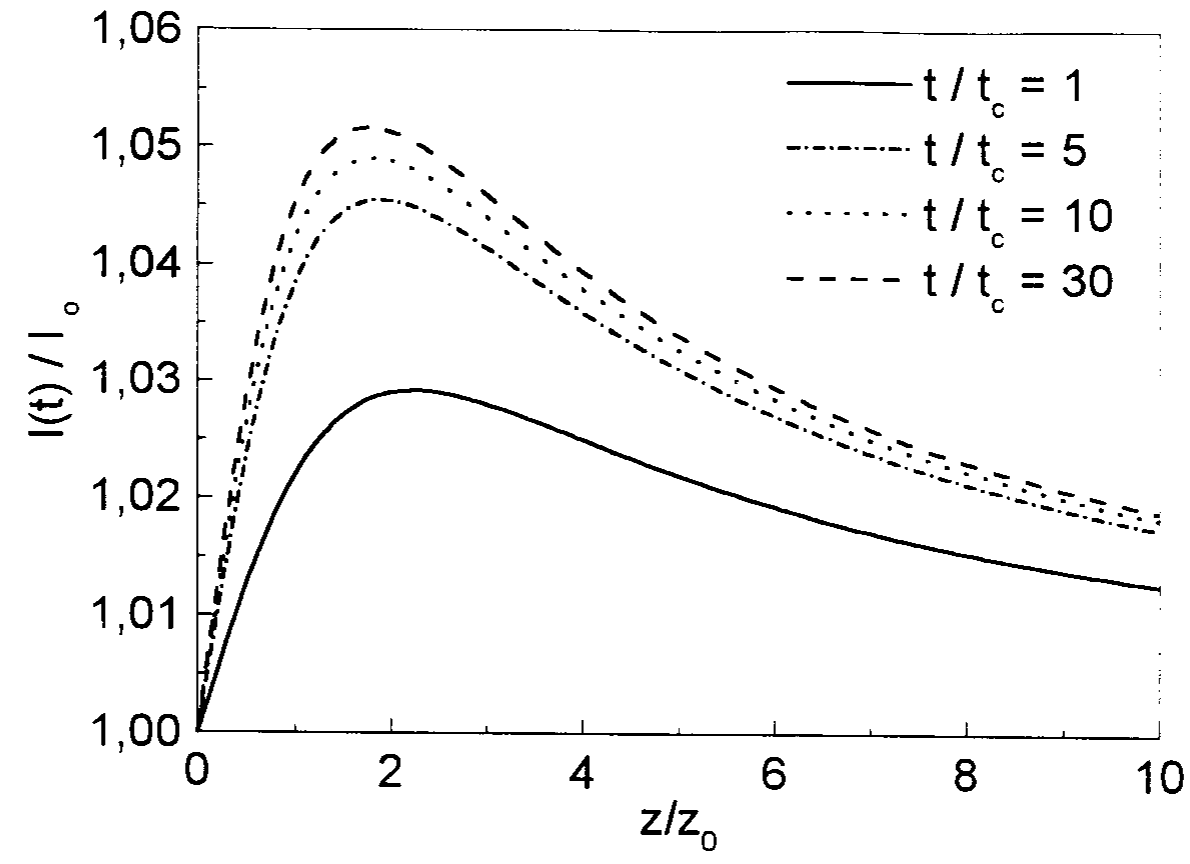

Figura 5.3 - Simulações do efeito de LT na Técnica Z-scan. 


\section{CAPÍTULO 6}

CONCLUSÃO 
Neste trabalho mostramos resultados em sólidos dopados obtidos através do uso da técnica de Lente Térmica na configuração descasada, Z-scan resolvida no tempo e analisamos a contribuição do efeito de Lente Térmica (LT) e efeito de Lente de População (LP) em sólidos dopados com íons $\mathrm{Nd}^{+3}$ e $\mathrm{Cr}^{+3}$. Apresentamos os primeiros resultados de medidas de $\Delta \alpha$ de ín $\mathrm{Nd}^{+3}$ em vidros fluoretos e cristais fluoretos dopados com $\mathrm{Cr}^{+3}$ Mostramos que estes sólidos tem um índice de refração não-linear $\mathrm{n}_{2}$ que é devido a diferença de polarizabilidade $\Delta \alpha$ entre os estados excitados e fundamental.

Com o uso da técnica de Z-scan resolvida no tempo mostramos que é possível, através da freqüência do chopper, distinguir temporalmente a contribuição do efeito de LP de LT, uma vez que o tempo de resposta característico da LP é $\tau_{0}$ e o tempo do efeito térmico é $t_{c}=w_{0}{ }^{2} / 4 D$. Entretanto, no caso do ZBLAN:Nd e outro vidros fluoretos observamos apenas o efeito térmico e não conseguimos observar o efeito não-linear. No cristal $\mathrm{SrAlF}_{5}: \mathrm{Cr}^{+3}$ observamos efeitos de saturação com intensidades aproximadamente 10 vezes menores que a intensidade de saturação $\left(\mathrm{I}_{\mathrm{s}}=330 \mathrm{KW} / \mathrm{cm}^{2}\right)$.

Nos cristais óxidos observamos que o efeito de Lente de População é muito maior que o de Lente Térmica. Estes cristais apresentam um alto valor da condutividade térmica $\mathrm{k}$, logo pudemos atribuir ao sinal de $\mathrm{Z}$-scan apenas o efeito de Lente de População. No entanto, os materiais fluoretos dopados com $\mathrm{Nd}^{+3}$ apresentam baixos valores de $\Delta \alpha$ e condutividade térmica $\mathrm{k}$ que aumenta o efeito de LT nestes materiais.

Mostramos que podemos relacionar os perfis de fase devido a LP e LT de tal maneira a podermos verificar qual destes efeitos é mais significativo. Ou seja: 


$$
\frac{\Delta \phi_{0}}{\Delta \phi_{\mathrm{th}}}=\left(\frac{7.85 \pi \lambda_{\mathrm{exc}}}{\varphi \mathrm{w}_{0}^{2} \mathrm{ch}}\right)\left(\frac{\mathrm{f}_{\mathrm{L}}^{2} \tau_{0} \Delta \alpha}{\mathrm{n}}\right)\left(\frac{\mathrm{K}}{\mathrm{ds} / \mathrm{dT}}\right)
$$

Como podemos observar, esta razão depende de $\mathrm{w}_{0}{ }^{2} \mathrm{e}$ isto implica em podermos alterar o arranjo experimental de maneira tal que se possa aumentar ou eliminar a contribuiçãu de um dos efeitos.

Portanto, mostramos que a técnica de Z-scan resolvida no tempo é uma ferramenta poderosa e confiável no estudo de efeitos não-lineares e térmicos, além de apresentar alta sensibilidade. Por esta técnica nós conseguimos medir pela primeira vez a diferença de polarizabilidade $\Delta \alpha$ do $\mathrm{SrAlF}_{5}: \mathrm{Cr}^{+3}[6]$. 


\section{APÊNDICE A}




\section{A.1. Tratamento Teórico da Técnica de Z-scan com dois feixes}

Generalizando o tratamento teórico da técnica de Z-scan para o caso onde se trabalha com dois feixe, um feixe de prova $\left(w_{p}\right)$ e um feixe de excitação $\left(w_{c}\right)$ que apresentam diâmetros diferentes o perfil de fase será dado por:

$$
\Delta \phi(\mathrm{y}, \mathrm{x})=\Delta \phi_{0}(\mathrm{x}) \exp \left[-2 \mathrm{my}^{2}\right]
$$

com

$$
\Delta \phi_{0}(\mathrm{x})=\frac{\Delta \Phi_{0}}{\left(1+\mathrm{x}^{2}\right)}
$$

e

$$
\Delta \Phi_{0}=\mathrm{kL}_{\mathrm{eff}} \mathrm{n}_{2}\left(\frac{2 \mathrm{P}}{\pi \mathrm{w}_{0}^{2}}\right)
$$

onde $m=\left(w_{p} / w_{e}\right)^{2}$

Resolvendo as integrais da Eqs. (3.15) e (3.16) temos:

$$
\begin{gathered}
H=\frac{\exp \left[-\beta^{2} 4(1+i x)\right]}{2(1+i x)} \\
\delta H=-i \Delta \phi_{0}\left(x_{\alpha}\right) \frac{\exp \left[-\beta^{2} 4(1+2 m+i x)\right]}{2(1+2 m+i x)}
\end{gathered}
$$


Analisando apenas o campo no centro $\left(r_{1}=0\right)$, temos $\beta=0$ e a função $\mathbf{J}_{0}(0)=1$, desta maneira as integrais (A.4) e (A.5) se tornam simples e temos como resultado:

$$
\begin{gathered}
H=\frac{1}{2(1+i x)} \\
\delta H=-i \Delta \phi_{0}(x) \frac{1}{2(1+2 m+i x)}
\end{gathered}
$$

A transmitância normalizada ao caso $\Delta \phi\left(\mathrm{r}_{0}\right)=0$, é dada por:

$$
\mathrm{T}=1+2 \mathrm{Re}\{\delta \mathrm{H} / \mathrm{H}\}
$$

Logo, utilizando as Eqs. (A.2), (A.6) e (A.7) em (A.8) teremos:

$$
\mathrm{T}\left(\mathrm{x}, \Delta \Phi_{0}\right)=1+\frac{4 \mathrm{x} \Delta \Phi_{0}}{\left(1+4 \mathrm{~m}^{2}+4 \mathrm{~m}+\mathrm{x}^{2}\right)\left(1+\mathrm{x}^{2}\right)}
$$

No capitulo 3 , comentamos que no caso não-linear a perturbação $\Delta \mathrm{n}(\mathrm{r})$ é localizada enquanto que no caso térmico ela se expande até valores de $\mathrm{r}$ bem maiores que $\mathrm{w}$, devido a difusão de calor. $\mathrm{Na}$ figura abaixo, fica claro que quando aumentamos o parâmetro $\mathrm{m}$, a sensibilidade da técnica de Z-scan diminui, contrário a técnica de Lente Térmica, que tem sua sensibilidade aumentada com o parâmetro $\mathrm{m}$ (Eq. 3.42). 


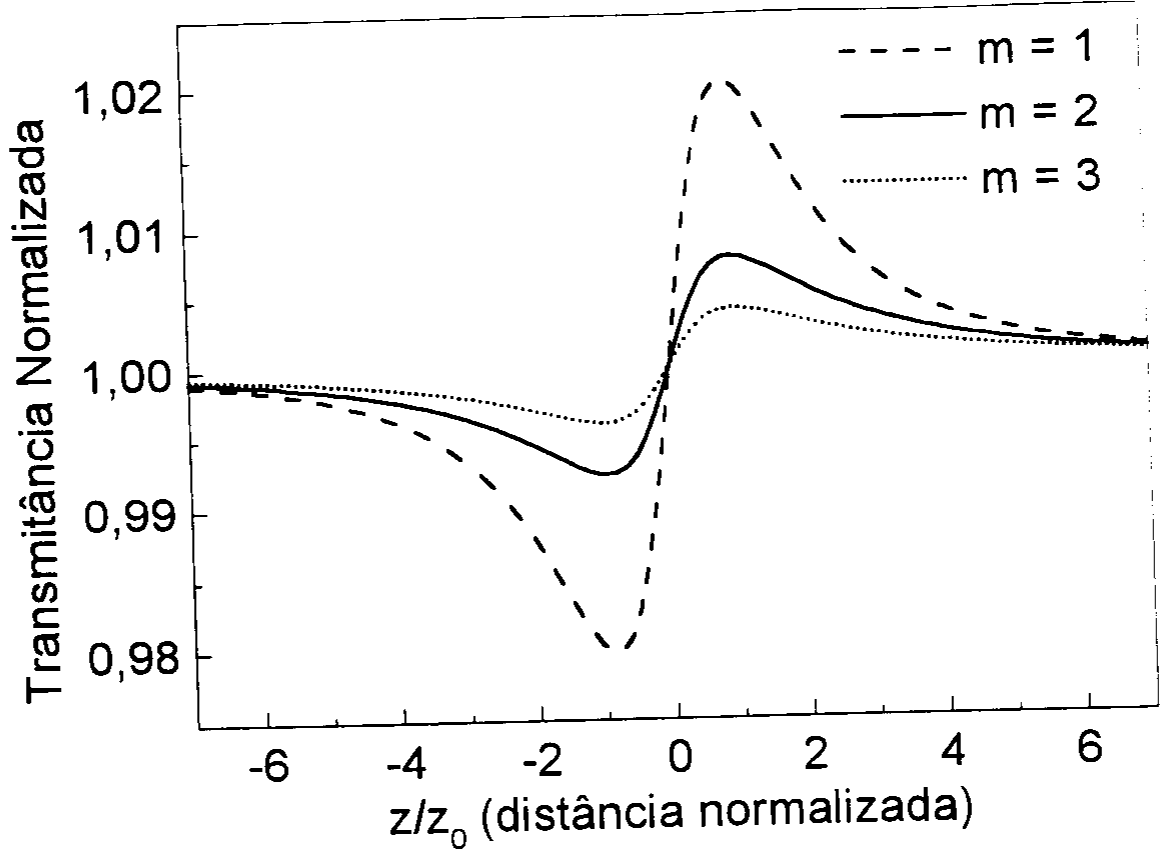

Figura A.1- Simulações teórica para a expressão de Z-scan com diferentes valores do parâmetro $\mathrm{m}$.

Este cálculo pode ser utilizado para estimar a contribuição do efeito de LP no experimento de LT de modo descasado. É interessante observar que a contribuição do efeito de LT aumenta com $m$ enquanto que o efeito de LP diminui vide Fig. A.1. Este fato pode ser usado para medir o efeito de LT em materiais que apresentam $\Delta \Phi_{0}>>$ $\Delta \phi_{\text {th }}$, que é o caso do rubi onde $\Delta \Phi_{0} / \Delta \phi_{\mathrm{th}}=995$ (vide Tabela 5.2) 
APÊNDICE B 


\section{B.1. Fator de correção}

Em qualquer medida experimental usando um "chopper" a resolução temporal está limitada ao tempo de subida e descida, ou seja, o tempo que a pá do chopper demora para interromper o feixe de luz. Isto provoca um arredondamento do sinal tal como mostra a Fig. B1 que compara o caso de um feixe com diâmetro grande (partes a e c) com o caso de diâmetro pequeno (partes b e d). Neste apêndice vou comentar como este tempo pode ser minimizado e como corrigir seu efeito no cálculo de $\mathrm{n}_{2}$.

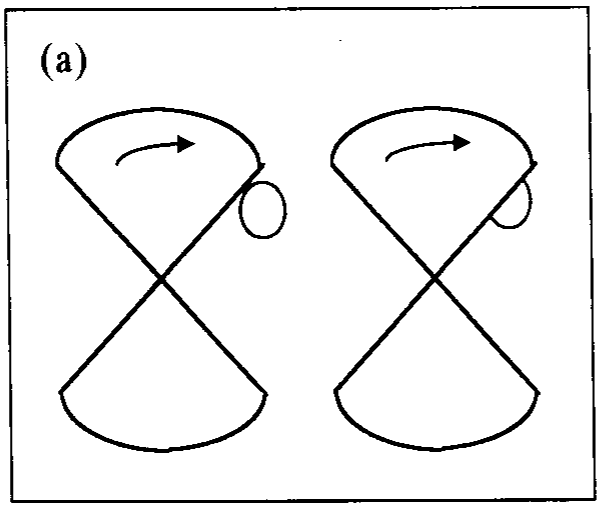

(c)

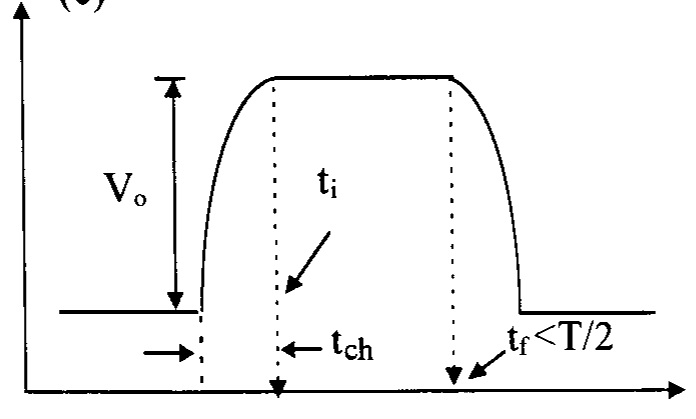

Tempo

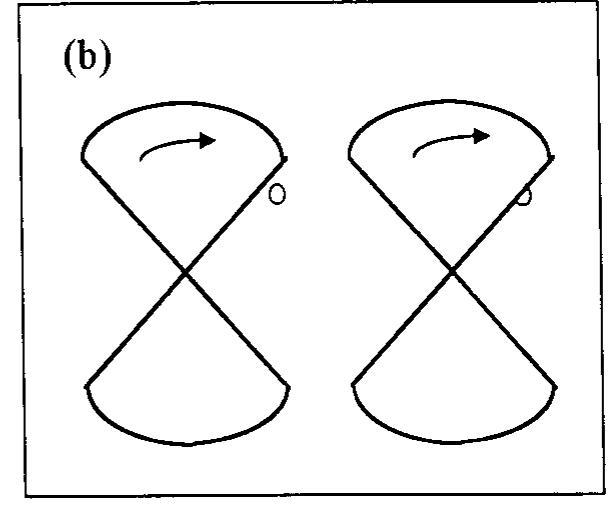

(d)

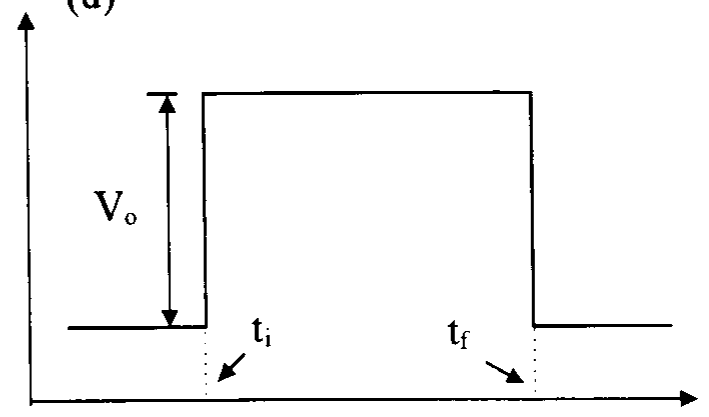

Tempo

Figura B.1 - A Figura (a) e (c) onde há a deformação na forma do sinal. A Figura (b) e (d) apresenta um caso ideal, sem deformação do sinal.

Este tempo é dado por $t_{c h}=v / 2 w$, onde $2 \mathrm{w}$ é o diâmetro do feixe do laser, $\mathrm{v}=\omega \mathrm{R}$ é a velocidade linear da pá do "chopper" na posição do centro do feixe a uma 
distância $\mathrm{R}$ do centro, e $\omega$ a velocidade angular da pá (o disco do "chopper"). Se o disco tem $n$ aberturas, a freqüência do sinal é $f=2 \pi n / \omega$, logo:

$$
t_{c h}=\frac{w}{\pi R} \frac{n}{f}
$$

onde podemos observar que $t_{c h}$ e w são grandezas diretamente proporcionais.

A Fig. B. 1 mostra um disco com 2 aberturas. Neste trabalho utilizamos discos $\operatorname{com} n=2$ e $n=6$, e $R=45 \mathrm{~mm}$. Sendo o raio do feixe do laser (w) na saida do laser igual a $750 \mu \mathrm{m}$ e usando uma freqüência de $826 \mathrm{~Hz}$ (o valor mais alto utilizado) da Eq. B. 1 temos um $t_{c h}=39 \mu$ s. Este valor representa uma resolução temporal muito baixa em alguns experimentos, por exemplo no caso do $\mathrm{SrAlF}_{5}: \mathrm{Cr}^{+3}$, onde o tempo de vida $\tau_{0} \sim 90 \mu \mathrm{s}$. Para obter um bom sinal da evolução temporal de $\Delta \mathrm{n}(\mathrm{t})$ é preciso ter $t_{c h}<<\tau_{0}$. Isto pode ser feito diminuindo o valor de $w$ na posição da pá do chopper através de um par de lentes de distância focal curta foco $\sim 10 \mathrm{~cm}$, vide Fig. 4.6 onde utilizamos $f=826 \mathrm{~Hz}$ e lentes de $10 \mathrm{~cm}$ de foco. Através de medidas de $z_{0}$ obtidos nos experimentos de Z-scan sabemos que estas lentes focalizam o feixe com $\mathrm{w} \sim 17$ $\mu \mathrm{m}, 44$ vezes menor que o valor original, o que nos dá então $t_{\mathrm{ch}} \sim 1 \mu \mathrm{s}$.

Consideraremos agora o cálculo da amplitude $\Delta \mathrm{V}$ do sinal quando a medida é feita em $t_{i}$ e $t_{f}$ (vide Fig. B.2). Supondo-se que o sinal é uma exponencial crescente com a amplitude $\Delta \mathrm{V}$ e tempo $\tau$ temos:

$$
V\left(t_{f}\right)=V_{0}+\Delta V\left(1-\exp \left[-t_{f} / \tau\right]\right)
$$




$$
V\left(t_{i}\right)=V_{0}+\Delta V\left(1-\exp \left[-t_{i} / \tau\right]\right)
$$

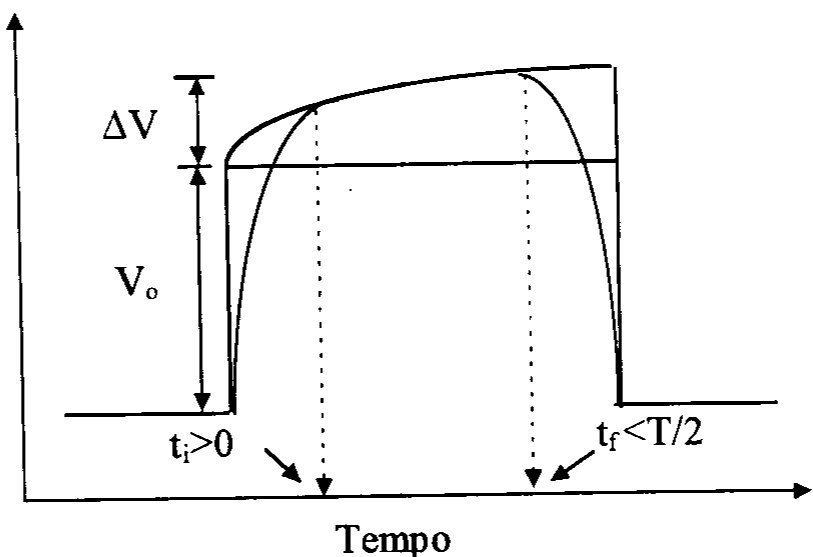

Figura B.2 - Forma do sinal com uma deformação devido ao tempo de abertura do chopper.

uma vez que no $\mathrm{Z}$-scan trabalha-se com amplitudes pequenas $\Delta \mathrm{V}<<\mathrm{V}_{0}$, logo de (B.2) e (B.3), temos:

$$
\frac{\mathrm{V}\left(\mathrm{t}_{\mathrm{f}}\right)}{\mathrm{V}\left(\mathrm{t}_{\mathrm{i}}\right)} \approx 1+\frac{\Delta \mathrm{V}}{\mathrm{V}_{0}}-\frac{\Delta \mathrm{V}}{\mathrm{V}_{0}} \exp \left[-\mathrm{t}_{\mathrm{f}} / \tau\right]-\frac{\Delta \mathrm{V}}{\mathrm{V}_{0}}+\frac{\Delta \mathrm{V}}{\mathrm{V}_{0}} \exp \left[-\mathrm{t}_{\mathrm{i}} / \tau\right]-\cdots
$$

portanto teremos:

$$
\frac{\Delta \mathrm{V}}{\mathrm{V}_{0}} \approx \frac{\frac{\mathrm{V}\left(\mathrm{t}_{\mathrm{f}}\right)}{\mathrm{V}\left(\mathrm{t}_{\mathrm{i}}\right)}-1}{\left.\left(\exp \left[-\mathrm{t}_{\mathrm{i} / \tau]}\right]-\exp \left[-\mathrm{t}_{\mathrm{f}}\right]\right]\right)}
$$

onde

$$
\frac{V\left(t_{f}\right)}{V\left(t_{i}\right)}-1=\Delta T
$$


Portanto, para considerar o efeito de $t_{\mathrm{i}} \neq 0$ e $\mathrm{t}_{\mathrm{f}}<\infty$ nos cálculos basta dividir $\mathrm{o}$ valor pelo denominador da expressão B.5. 
APÊNDICE C 


\section{C.1. Cálculo da expressão que considera o efeito de $L T$ e $L P$.}

A superposição dos efeitos de LT e LP é um problema geral da técnica de Zscan, não só no caso dos sólidos mas também em líquidos, polímeros, etc. Com o intuito de verificar até que ponto conseguimos distinguir temporalmente a contribuição deste efeitos, elaboramos um modelo teórico que considera a ação simultânea destes Jois efeitos. A expressão para o Z-scan é dado pela Eq. (3.27):

$$
\mathrm{T}\left(\mathrm{x}, \Delta \Phi_{0}\right)=1+\frac{4 \mathrm{x} \Delta \Phi_{0}}{\left(9+\mathrm{x}^{2}\right)\left(1+\mathrm{x}^{2}\right)}
$$

e a expressão para a Lente Térmica normalizada, para o caso de um único feixe $(\mathrm{m}=1)$ é dada pela Eq. $(3.42)$ :

$$
\frac{I(t)}{I(0)}=\left\{1-\frac{\theta}{2} \operatorname{atan}\left[\frac{2 x}{\left[9+x^{2}\right] \frac{t_{c}}{2 t}+3+x^{2}}\right]\right\}^{2}
$$

expandindo a Eq. (C.2) e tomando apenas o termo de primeira ordem para $\theta$, temos:

$$
1-\theta \operatorname{atan}\left[\frac{2 x}{\left[9+x^{2}\right] \frac{t_{c}}{2 t}+3+x^{2}}\right]
$$


agora considerando as Eqs. (C.1) e (C.3) temos como resultado final:

$$
\Delta T=\frac{4 \Delta \Phi_{0} x}{\left(1+x^{2}\right)\left(9+x^{2}\right)}+\theta \tan ^{-1}\left(\frac{2 x}{\left(9+x^{2}\right) \frac{t_{c}}{2 t}+3+x^{2}}\right)
$$




\section{REFERÊNCIAS}


[1] V. PLLA, IMPINNISI, P.R. and CATUNDA, T., "Measurement of Saturation Intensity in Ion Doped Solids by Transient Nonlinear Refraction," Appl. Phys. Lett., vol. 70, pp. 817, 1997.

[2] R. POWELL, S. PAYNE, L. CHASE and G. WILKE, "Four-wave mixing of $\mathrm{Nd}^{+3}$ doped crystals and glasses," Phys. Rev. B, vol. 41, pp. 8593, 1990.

[3] M. L. BAESSO, SHEN, J. and SNOOK, R. D., "Mode Mismatched thermal Lens Determination of Temperature Coefficientc of optical path Length in Soda Lime Glass at Different Wavelenghts," J. Appl. Phys., vol. 75, pp. 3732$3748,1994$.

[4] M. SEIK-BAHAE, SAID, A. A., WEI, T., HAGAN, D. and ETRYLAND, E. W. V., "Sensitive Measurement of Optical Nonlinearities Using a Single Beam," IEEE J. Quantum Electronics, vol. 26, pp. 760-769, 1990.

[5] A. A. ANDRADE, CATUNDA, T.; LEBUllENGER, R.; HERNANDES, A.C. and BAESSO, M.L., "Time-resolved study of thermal and electronic nonlinearities in $\mathrm{Nd}^{+3}$ doped fluoride glasses," Electronics Letters, vol. 38, pp. $117-119,1998$.

[6] A. A. ANDRADE, TENÓRIO, E., CATUNDA, T., BAESSO, M.L. CASSANHO, A. and JENSSEN, H, P., "The discrimination between electronic and thermal contributions to the nonlinear refractive index of SrAlF5:Cr ${ }^{+3}$," submetido a publicação no JOSA B, 1998.

[7] W. KOECHNER, Solide-State Laser engineering. New York: Spring Verbag, 1988.

[8] H. P. JENSSEN, LAI, S. T., "Tunable-laser characteristics and spectroscopic properties of SrAlF5:Cr ${ }^{+3}$," J. Opt. Soc. Am. B, vol. 3, pp. 115, 1986. 
[9] L. C. OLIVEIRA, ZILIO, S. C., "Single-beam time-resolved Z-scan measurements of slow absorbers," Appl. Phys. Lett., vol. 65, pp. 1-3, 1994

[10] L. C. OLIVEIRA, CATUNDA, T. and ZILIO, S. C., "Saturation effects in Z-scan measurements," Jnp. J. appl. Phys., vol. 35, pp. 25-28, 1996.

[11] T. CATUNDA, BAESSO, M. L., MESSADDEQ, Y. and AEGERTER, M., "Measurement of the complex nonlinear refractive index of $\mathrm{Er}$ and $\mathrm{Nd}$ doped glasses," Jounal of Non-Crystalline Sclids, vol. 213\&214, pp. 225-230, 1997.

[12] S. C. WEAVER, PAYNE, S., "Determination of excited-state polarizabilities of $\mathrm{Cr}^{+3}$ doped materials by degenerate four-wave mixing," Phys. Rev. B, vol. 40, pp. 10727, 1989

[13] T. CATUNDA, CURY, L. A., "Transverse self-phase modulation in Ruby and GdalO3:Cr ${ }^{+3}$ crystals," J. Opt. Soc. Am. B, vol. 7, pp. 1445-1455, 1990.

[14] C. R. MENDONÇA, B. J. COSTA, Y., MESSADDEQ and S. C. ZÍLIO, “Optical properties of chromium-doped fluoroindate glasses," Physical Review B, vol. 56, pp. 2483-2487, 1997.

[15] R. POWELL, PAYNE, S., "Dispersion Effects in Four-Wave Mixing Measurements of Ion in Solids," Optics Letters, vol. 15, pp. 1233-1235, 1990.

[16] H. J. G. EICHLER, P., and POHL,D. W., Laser-Induced Dynamic Gratings, vol. 50. Berlin: Spring - Verlag, 1986.

[17] M. SHEIK-BAHAE, SAID, A. A. and STRYLAND, E. W. V., 'High sensitivity single beam $\mathrm{n}_{2}$ measuremente," Opt. Lett., vol. 14, pp. 955-957, 1989. 
[18] M. L. BAESSO, SHEN, J. and SNOOK, R. D., "Time-resolved thermal lens measurement of thermal diffusivity of soda-lime glass," Chemical Physics Letters, vol. 197, pp. 255-258, 1992.

[19] H. L. FRAGNito, E. PALANGE., "Medidas de $\mathbf{n}_{2}$ com a técnica de Z-Scan," presented at Escola de Verão Jorge André Swieca de Óptica Quântica e Óptica Não-linear, IFGW- UNICAMP, Campinas, 1994.

[20] S. J. SHELDON, KNIGHT, L. V. and THORNE, J. M., 'Laser-induced thermal lens effect: a new theoretical model," Appl. Opt., vol. 21, pp. 1663$1669,1982$.

[21] J. SHEN, SNOOK, R. D., "A radial finite model of thermal lens spectrometry and the influence of sample radius upon the validity of the radial infinite model," J. Appl. Phys., vol. 73, pp. 5286-5288, 1993.

[22] A. P. STEPHEM, SMITH, L. K., BEACH, J. R. et al., 'Properties of Cr:LiSrAlF 6 crystals for laser operation," Applied Optics, vol. 33, pp. 20, 1994.

[23] S. UEMURA, MIYAZAKI, K. "Thermal Characteristics of a ContinuousWave Cr:LiSAF Laser," Jpn. J. Appl. Phys., vol. 36, pp. 4312-4315, 1997.

[24] F. BALEMBOIS, FAOCOS, F., FRÉDÉRIQUE, K., FRÉDÉRIC, D., GEORGES, P. and BRUN, A., "Theoretical and Experimental Investigations of Small-Signal Gain for a Diode-Pumped Q-Switched Cr:LiSAF Laser," IEEE Journal of Quantum Electronics, vol. 33, pp. 269-278, 1997.

[25] R. POWELl, PAYNE, S. A. PAYNE, L. L. and WILKE, G. D., "Index of Refraction Change in Optically Pumped Solid Laser Materials," Opt. Lett., vol. 14, pp. $1204-1207,1989$. 
[26] T. IZUMITANI, YAMASHITA, T., TOKIDA, M., MINURA, K. and TAJIMA, H., Mat, Sci. Forum, vol. 19, pp. 19-20, 1987.

[27] T. CATUNDA, "Estudo de índice de refração não-linear e efeitos fototérmicos em sólidos dopados," Tese apresentada ao IFSC. São Carlos: USP, 1989.

[28] A. MARCANO, HERNÁNDEZ, F.E., "Two-color near-field eclipsing Z-scan techinique for the determination of nonlinear refraction," J. Opt. Soc. Am. B, vol. 12, pp. 3363-3367, 1997.

[29] M. TERAZIMA, "Ultrafast transient Kerr lens in soltion detected by the dualbeam thermal-lens method," Optics Letters, vol. 20, pp. 25-27, 1995.

[30] M. TERAZINA, HARA, T. and HIROTA, N., "Separation of Transient Absoption and Population Lens Effect from the "Thermal Lens" Signal," J. Phys. Chem., vol. 97, pp. 13668-13672, 1993.

[31] C. R. MENDONÇA, COSTA, M. M., GIACOMETTI, J. A., NUNES, F. D. and ZILIO, S. C., "Nonlinear refractive indices of polystyrene films doped with azobenzene dye Disperse Red 1," Electronics Letters, vol. 38, pp. 116$117,1998$.

[32] M. L. BAESSO, BENTO, A.C., ANDRADE, A. A., CATUNDA, T, SAMPAIO, J. and GAMA, S., 'Neodymium concentration dependence of the thermo-optical properties inlow silica calcium aluminate glasses," J. NonCryst. Solids, vol. 219, pp. 165-169, 1997. 
ANEXOS

\section{TRABALHOS PUBLICADOS}


erence [5]. Defining $\Delta R_{p}$, as the difference of $R(z, n)$ for the peak and valley positions, we can measure the dependence of $\Delta R_{p}$ on $f$, shown in Fig. $2 b$. The relationship between the thermal response time at the peak (or valley) position $z_{p}$ and the modulation frequency is:

$$
T_{\mathrm{th}}=\frac{w\left(z_{p}\right)^{2}}{4 D}=\frac{w_{0}^{2}}{4 D}\left(1+\left[\frac{z_{p}(f)}{z_{0}}\right]^{2}\right)
$$

where $w_{0}$ is the beam waist radius and $z_{p}(f)$ is the frequencydependent peak position, which is experimentally available. Using the expression for $R(z f)$ with the thermal time given by eqn. 1, we can fit the experimental data, as shown by the solid curve of Fig. $2 b$. We obtained $n_{2}=-9.3 \times 10^{-9} \mathrm{~cm}^{2} / \mathrm{W}$ and $D=6.6 \times 10^{-1} \mathrm{~cm}^{2} / \mathrm{s}$, which is in good agreement with values presented in the literature for a pure polystyrene film.

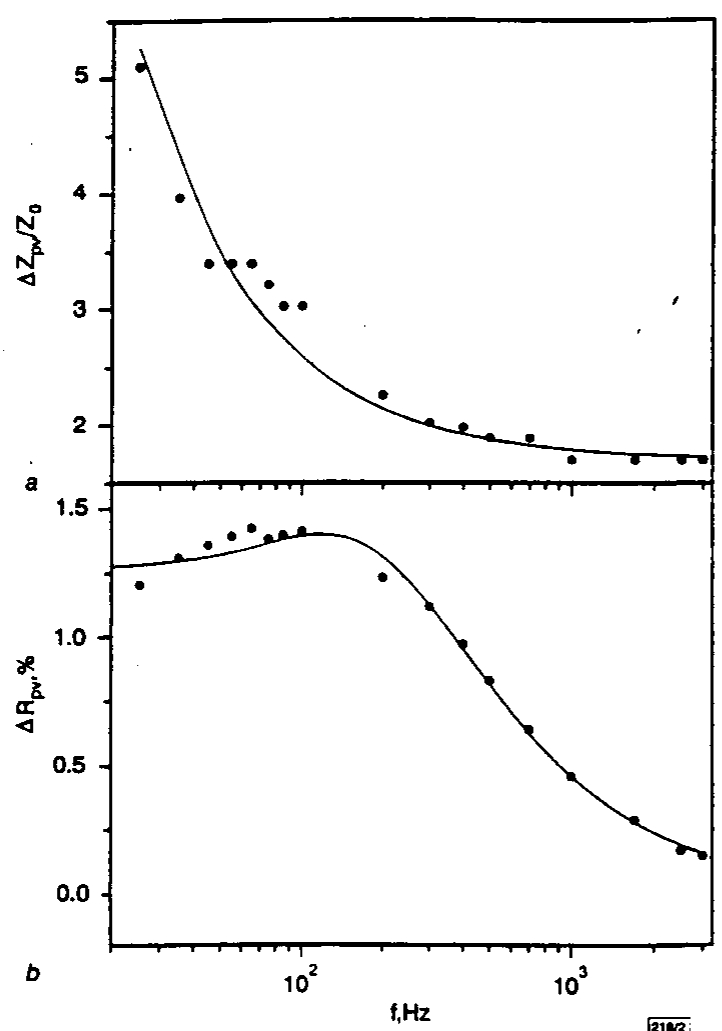

Fig. 2 Dependence of $\Delta Z_{p v}$ on chopper frequency and $\Delta R_{p v}$ against modulation frequency

a $\Delta Z_{n v}$ against chopper frequency

theoretical fit with function $\mathrm{l} / f+$ constan

against modulation frequency

theoretical fit provided by eqn. 4 of [5]

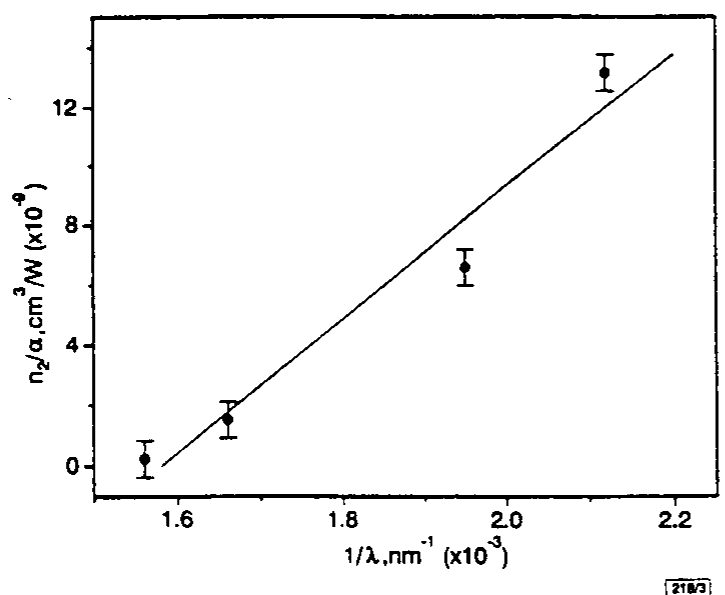

Fig. 3 Dependence of ratio $n_{2} / \alpha$ on the wavenumber $(1 / \lambda)$

We have also carried out measurements as a function of the wavelength. We carefully determined the linear absorption coeffi- cient $\alpha$ at each wavelength used, and obtained the ratio $\mathrm{m} / \alpha$ shown in Fig. 3. This ratio is enhanced in the blue region where the energy of the incident photon increases, as expected for a thermal nonlinearity. -

Conchisions: In summary, we have measured nonlinear refractive indices in a polystyrene film containing DR1, using the Z-scan technique with Fourier analysis. We observed a dependence of $\Delta Z_{\mathrm{m}}$ on the chopper frequency, which is characteristic of thermal effects. The value obtained for the thermal diffusivity, and the behaviour of the ratio $n / \alpha$ as a function of the wavelength, supports the thermal origin of the signal. Although the excitation was carried out in a pre-resonant condition, the small residual absorption in the wing of the band, not observed with a conventional spectrometer, is enough to give rise to the thermal nonlinearity. We believe that the same situation occurs for the samples studied in [4].

Acknowledgments: This research was supported by Fundação de Amparo à Pesquisa do Estado de São Paulo (FAPESP) and the program PRONEX, grant 4.1.96.0935.00

@ IEE 1998

26 August 1997

Electronics Letters Online No: 19980010

C.R. Mendonça, M.M. Costa. J.A. Giacometti, F.D. Nunes and S.C. Zilio (Departamento de Física e Ciéncia dos Materiais. Instituto de Fisica de São Carlos, Universidade
13560-907 São Carlos, SP. Brazin

\section{References}

BLOEMBERGEN. N.: 'Nonlinear optics of polymers: fundamental and applications', J. Nonlinear Opt. Phys. Mater., 1996. 5, pp. 1-7

2 CHEMLA. D.S., and ZYSS. J. (Eds.): 'Nonlinear optical properties of organic molecules and crystals' 2, (Academic Press. NY, 1987)

3 PRASAD. P.N., and WILLIAMS. D.J.: 'Introduction to nonlinear optical effects in molecules and polymers' (Wiley, NY, 1991)

4 ZHANG. Z.X., OIU, W., PUN. E.Y.B., CHUNG. P.S., and SHEN. Y.Q.: 'Doped poiymer films with high nonlinear refractive indices', Electron. Lett., 1996, 32, pp. 129-130

5 MENDONCA. C.R., MISOGUTI. L., and ZiLIO. S.C.: 'Z-scan measurements with Fourier analysis in ion-doped solids', Appl. Phys. Lett. 1997, 71, (15), pp. 2094-2096

6 SHEIK-BAHAE M. SAID. A.A. and VAN STRYLAND. E.W.: 'High. sensitivity, single beam $n$, measurements', Opt. Lett. 1989. 14. pp. 995-957

7 Sheik-bahae. M.. Said. A.A., wei. T., hagan. D.J. and vaN STRYLAND. E.W.: 'Sensitive measurement of optical nonlinearities using a single beam', IEEE J. Quantum Electron., 1990. QE-26. pp. $760-769$

\section{Time-resolved study of thermal and electronic nonlinearities in $\mathrm{Nd}^{+3}$ doped fluoride glasses}

A.A. Andrade. T. Catunda, R. Lebullenger.

A.C. Hernandes and M.L. Baesso

Using a time resolved Z-scan technique, the authors distinguished electronic (population lens) and thermal nonlinearites. The first measurements of the polarisability difference $\Delta \alpha$ between excited $\left({ }^{4} F_{3 / 2}\right)$ and ground $\left({ }^{4} I_{9 / 2}\right)$ states of $\mathrm{Nd}^{+3}$ ions in fluoride giasses are presented. A value of $\Delta \alpha=1.6 \times 10^{-26} \mathrm{~cm}^{3}$ was obtained in fluorindate glass and $\Delta \alpha<0.2 \times 10^{-26} \mathrm{~cm}^{3}$ was estimated for ZBLAN

The noninear properties of ion-doped solids have been studied by different techniques. Particularly, solids doped with $\mathrm{Cr}^{+3}$ and $\mathrm{Nd}^{*}$ have been much investigated due to their applications as laser materials $[1-6]$. This noniinearity can be used for all optical switching and transient gratings in fibres [4]. In these materials. the nonlinearity originates from the population of the dopant ion metastable state, which has a complex susceptibility different from that of the ground state. The real part of the noniinear refractive

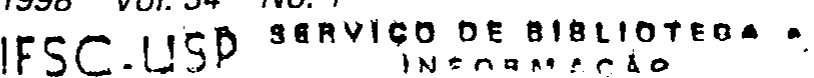


Inaex is proportional to the polarisability difference $\Delta \alpha$ between excited and ground states; the imaginary part is proportional to the absorption cross-section difference $\Delta \sigma$ between excited and ground states. We call this process the population lens effect (PL) Most of these solids present a nonlinearity whose real part is one order of magnitude greater than the imaginary part. Usually, part of the excited state decay is non-radiative so the Usually, part sample and an optical path change is established laser heats the temperature coefficient of the optical path; ds/dT, which causes the so-called thermal-lens (TL) effect. As in other materials, the Zscan technique [7] has been shown to be the best one for the study of ion-doped solids which have a study milliseconds) [1 - 3]. In fluoride glasses. $\Delta \alpha$ is usually very small and it has been observed that the PL effect is smaller than the TL effect [2]. In this Letter, we show that it is possible to temporally distinguish these two effects. and we present the first $\Delta \alpha$ measurements for $\mathrm{Nd}^{+3}$ doped fluoride glasses.

In the first-order (for $I \ll I_{s}$ ), the excited state population is given by $N_{e x}=N_{o} I / I_{s}$, where $N_{o}$ is the total ion concentration $I$ is the laser intensity. The saturation intensity is given by $\hbar \omega / \sigma \tau_{0}$, where $\hbar \omega$ is the pump phon state lifetime and $\sigma$ is the absorption energy, $\tau_{o}$ is the excited induced induced phase shift is given by $\Delta \phi_{\text {pop }}=(2 \pi \lambda) \Delta n_{\text {pop }}^{\prime} L$ and can be
written as [1]

$$
\Delta \phi_{\text {pop }}=\left(4 \pi^{2} f_{L}^{2} / \lambda n_{o}\right) \Delta \alpha L N_{o} I_{o} / I_{s}
$$

where $f_{L}=\left(n_{0}^{2}+2\right) / 3$ is the Lorenz local field correction factor $\Delta \alpha$ is the polarisability difference between excited and ground states of the dopant ion, $I_{0}=2 P / \pi w_{0}^{2}$ is the on-axis intensity Gaussian $T^{2} M_{00}$ profile, $w_{0}$ is the beam radius at focus, and $P$ is the laser power.

The thermal lens (TL) effect is caused by deposition of heat. via a non-radiative decay process after the laser energy has been absorbed by the sample. The TL effect can be treated through the calculation of the temporal evolution of the sample temperature profile $\Delta T(r, t)$, caused by a Gaussian beam. The IL transient signal amplitude is proportional to its phase shift given by [2]

$$
\Delta \phi_{t h}=\frac{P . A L}{K^{*} \lambda_{p}} \varphi \frac{d s}{d T}
$$

where $P$ is the excitation laser power. $A$ is the absorption coefficient. $K$ is the thermal conductivity, $\lambda_{n}$ is the probe beam wavelength. $L$ is the sample length and $\varphi$ is the fraction of absorbed energy converted into heat per photon. The TL signal response
time is given by

$$
t_{c}=\frac{w^{2}}{4 D}
$$

where $D=K / \rho C$ is the thermal diffusivity, $\rho$ is the density, $C$ is the specific heat and $w$ is the excitation beam radius. The propagation of a $T_{E M}$ Gaussian laser beam through a phase profile due to PL and/or TL is affected. resulting in either beam spreading with a reduction in its on-axis intensity, or beam focusing with an increase in on-axis intensity. By measuring the beam on-axis intensity in the far-field. the thermo-optical and/or nonlinear properties of the sample can be obtained. The beam behavinear properties focusing) depends on the sign of $\Delta \phi$ and the tive to focus. This is the main ides lens [2] techniques which main idea of both Z-scan [7] and therma ple, are very sensible. When the non

When the noniinearity response time is slow, due to a long-lived used in transient response measurements can be imperfections fo eiiminate parasitic linear effects (like surface imperfections, for instance), allowing measurements of very small phase shifts [3]. Assuming that at $t=0$ the laser is turned on. from the rate equations we obtain the ion excited state population $N_{e x} \propto$ $N_{0}\left(1-e^{-1 / \tau}\right)\left(I / I_{s}\right)$ with $\tau^{-1}=\tau_{o}^{-1}\left(1+I / I_{s}\right)[1]$

The experiment was performed with an $\mathrm{Ar}^{*}$ laser at $\lambda=515 \mathrm{~nm}$ in a $1 \% \mathrm{Nd}$ doped InSBZnGdN fluorindate laser at $\lambda=515 \mathrm{~nm}$ $2.5 \times 10^{5} \mathrm{~W} / \mathrm{cm}^{2}\left(\sigma=3.2 \times 10^{-21} \mathrm{~cm}^{2}\right.$ and $\tau^{2}=470 \mu$ [2], where $I_{s}=$ single beam time-resolved $Z$ - $\mathrm{scm}^{2}$ and $\left.\tau_{0}=470 \mu \mathrm{s}\right)$. Fig. 1 shows different different chopper frequencies in order to discriminate between PI and TL effects in $\mathrm{Nd}^{+3}$ doped fluorindate glass [2] nent the PL effect is faster han quencies the characteristic Z-scan curve for at high chopper frewas observed and attributed to the PL effect. The PL curve shown is already normalised by the open aperture $Z$ The PL curve shown state absorption) as is usually. done in this technique [1]. In the PL curve the computer was set to measure at $t \approx 300 \mu \mathrm{s}$ where $\Delta \phi_{\text {pop }}$ is -0.4 of its stationary value given by eqn. 1 .

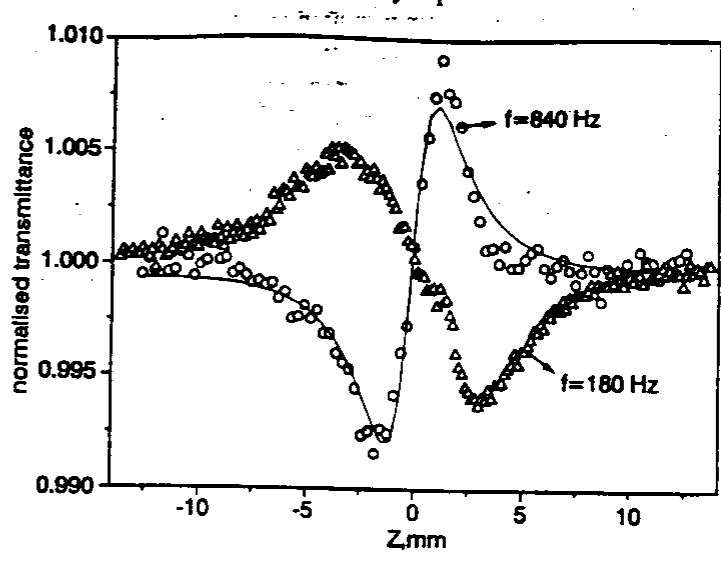

Fig. 1 Time-resolved $\mathrm{Z}$-scan of $\mathrm{Nd}^{+3}$ doped fluorindate glass

$\triangle$ Chopper frequency $f=90 \mathrm{~Hz}, P=0.23 \mathrm{~W}$
$Q f=840 \mathrm{~Hz}, P=0.187 \mathrm{~W}$

At lower chopper frequencies (indicated by PL in Fig. 1) the curve is inverted, indicating that $\Delta n<0$ and consequently that $d s /$ $d T<0$, as usually observed in fluoride glasses. The distance between the peak and the valley is doubled $\left(\Delta T_{p-v} \simeq 3.4 z_{o}\right)$ as expected for a TL Z-scan [2]

We also measured $\mathrm{Nd}^{+3}$ doped ZBLAN glass. However, in this sample, the electronic nonilinearity is too small compared to the thermal one, so we could only estimate $\Delta \alpha<0.2 \times 10^{-26} \mathrm{~cm}^{3}$.

Using a four-wave-mixing technique. Powell et al. [6] measured $\Delta \alpha$ in several kinds of host material (crystal and glasses). They observed that in fluoride crystals and fluorophosphate glasses. $\Delta \alpha$ is up to one order of magnitude smaller than in oxides. From our PL data. we calculated $\Delta \alpha \sim 1.6 \times 10^{-26} \mathrm{~cm}^{3}$, which is similar to the values found for fluoride crystals and fluorophosphate glasses. Most of the active ion doped solids simultaneously present both $\mathrm{PL}$ and TL effects [5]. Ion doped fluorides usually have low $\Delta \alpha$ and. consequently a low PL effect. We have shown that even when $\Delta \phi_{\mathrm{ih}}>\Delta \phi_{\mathrm{p}}$, these two effects can be distinguished temporally by measuring with appropriate chopper frequencies. The same procedure has been used before to distinguish the nonlinear effect of the two different sites of $\mathrm{Cr}^{-3}$ in alexandrite [1].

Acknowledgments: This work was supported by FAPESP. CAPES and $\mathrm{CNPq}$.

(C) IEE 1998

Electronics Letters Online No: 19980056

13 October 1997

A.A. Andrade. T. Catunda, R. Lebullenger and A.C. Hernandes (Instituto de Física de Süo Paulo. Universidade de São Carlos. C.P. 369

M.L. Baesso (Departamento de Fisica. Universidade Estadual de Maringá, Av. Colombo 5790. 87020-900, Maringá, PR. Bra=il)

\section{References}

1 PILla. $V$. IMPINNISI. P.R. and CATUNDA. T.: 'Measurement of saturation intensity in ion doped solids by transient noninear refraction', Appl. Phy.s. Lett., 1997, 70, pp. 817-819

2 CATUNDA. T., BAESSO, M.L.. MESSADDEO. Y. and AEGERTER. M.A 'Time-resolved Z-scan and thermal lens measurements in $\mathrm{Er}^{+3}$ and $\mathrm{Nd}^{+3}$ doped giasses'. J. Non-Cryst. Solids. 1997. 213\&214. pp. 225-

3 OLIVEIRA. L.C., and ZiLIO. S.C.: 'Single-beam time-resolved Z-scan measurements of slow absorbers', Appl. Phy.s. Lett. 1994 65. Pp. $2121-2123$

4 SADOWSKI. R.M., DIGONNET. M.J., PANTELL. R.H., and SHAW. H.J. 'Microsecond optical-optical switching in a neodymium-doped

HOMMERICH. U. OPI. Lell. 1993. 11. pp. 927-929

induced lensing effects in NRAUSS.E., and YEN. W.M.: 'Optically induced lensing effects in $\mathrm{Nd}^{+3}$ doped laser glass measured by

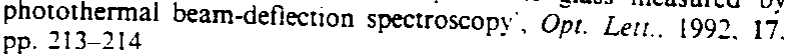


of $\mathrm{Nd}^{+3}$ doped crystals and glasses?. Phys. Rev. B, 1990, 41, pp. 8593-8602

7 SHEIK-BAHAE, M. SAID. A.A., WEI. T. MAGAN, D., and STRYLAND. E.W.V.: 'Sensitive measurement of optical nonlinearities using a single beam', IEEE J. Quantum Electron., 1990, QE-26, pp. $760-769$

\section{$550 \mathrm{GHz}$ bandwidth photodetector on low- temperature grown molecular-beam epitaxial GaAs}

\section{P. Kordoš, A. Förster, M. Marso and F. Rüders}

The authors demonstrate that a $550 \mathrm{GHz}$ bandwidth photodetector can be fabricated on low-temperature grown MBE GaAs. The pulse response shows 0.4 and $0.6 \mathrm{ps}$ rise and fall times, respectively. The bandwidth is in agreement with a value calculated using a carrier lifetime of $0.2 \mathrm{ps}$, measured by calculated using a carrier lifetime of $0.2 \mathrm{ps}$, measured by
femtosecond time-resolved reflectivity, and a capacitance of $0.014 \mathrm{fF} / \mathrm{um}^{2}$. determined from microwave measurements. The device bandwidth is RC limited.

Introduction: Molecular-beam epitaxial GaAs grown at a low substrate temperature $\left(150-350^{\circ} \mathrm{C}\right.$, called LT GaAs. is nonstoichiometric ( $\leq 1.5$ at $\%$ of excess arsenic) and highly resistive (s $10^{7} \Omega \mathrm{cm}$ ) [1]. LT GaAs is useful in microelectronics, as a buffer to substantially reduce shor-channel effects in MESFETs, or as an insulator in MISFETs. The I-V product of $16.8 \mathrm{~W} / \mathrm{mm}$ was obtained on LT GaAs based MISFETs [2]. Owing to an extremely high density of defects (up to $10^{18} \mathrm{~cm}^{-3}$ ) the LT GaAs should be favourable material for high-speed optoelectronic applications [3] and metal-semiconductor-metal (MSM) photoconductive switches with sub-picoseconds rise time, and a few picoseconds FWHM were reported $[4,5]$. However, in these devices. after a fast initial decay of the pulse response, an additional slower decay is observed. which degrades the bandwidth of a photodetector (PD) This effect is attributed to the different trapping and recombination times in LT GaAs [6]. The properties of LT GaAs are critically dependent on growth and annealing conditions. The main parameter here is the substrate temperature. The density of defects increases strongly if the substrate temperature decreases. In addition, growth should be limited to a thickness less than the critical thickness for the formation of the pyramidal defects and polycrystalline material [1]. The critical thickness decreases strongly along with decrease in the substrate temperature. For example. for $T_{3}=$ $200^{\circ} \mathrm{C}$ the critical thickness is only $0.6 \mu \mathrm{m}$. Conversely, the absorption length in $\mathrm{GaAs}$ is $1 / \alpha=1 \mu \mathrm{m}$. where $\alpha$ is the absorption coefficient at $0.8 \mu \mathrm{m}$ wavelength. Therefore. in order to optimise the properties of LT GaAs PDs. detailed analysis of all parameters which can influence the material [7], as well as the device behaviour, needs to be performed.

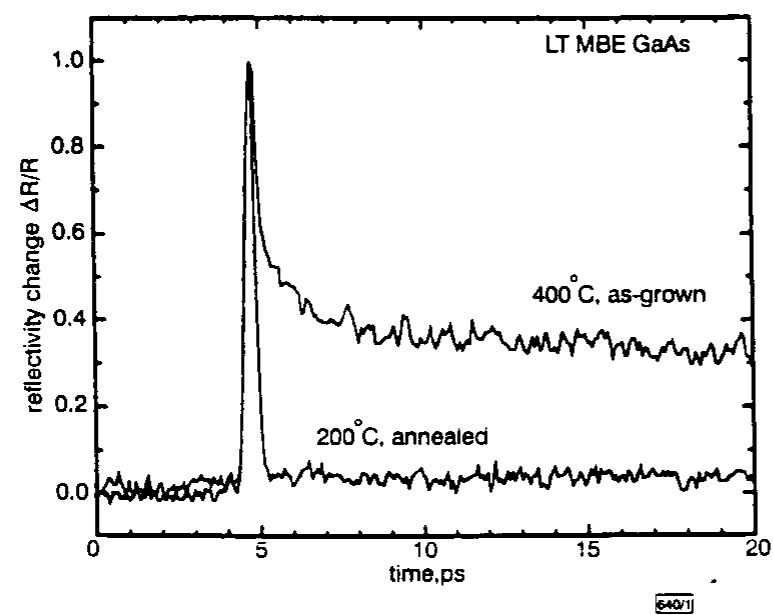

Fig. 1 Reflectivur change (normalised) in LT GaAs grown at different substrate temperatures hat has faster puise response (rise and fall times of 0.4 and $0.6 \mathrm{ps}$, ousty reported. The observed bandwidth is in agrement with value calculated using measured data about carrier lifetime and the microwave capacitance.

Device fabrication: The LT GaAs layers were grown in a Varian Mod GEN II MBE system on semi-insulating GaAs wafers. An As $/ \mathrm{Ga}$ beam-equivalent pressure ratio of 19 , and a growth rate of lum were used to orow 2 um thick GaAs layers. Wafers with a GaAs layer were cleaved and a part was annealed at $600^{\circ} \mathrm{C}$ for $10 \mathrm{~min}$ by rapid thermal processing under local As overpressure. PD fabrication consisted of optical lithography, metal evaporation and lift-off processes. Interdigitated finger electrodes with fingerspacing $s$ and -width $w$ of $1-3 \mu \mathrm{m}(s+w=3,3.5$ and $4 \mu \mathrm{m})$ were used. The devices had an area of $20 \times 20$ and $50 \times 50 \mu \mathrm{m}^{2}$.

Results and discussion: At first the lifetime of photogenerated carriers in LT GaAs was studied using femtosecond time-resolved reflectivity measurements. A $\mathrm{Ti}: \mathrm{Al}_{2} \mathrm{O}_{3}$ laser, producing $100 \mathrm{fs}$ FWHM pulses at $700 \mathrm{~nm}(1.77 \mathrm{eV})$ with a repetition rate of $76 \mathrm{MHz}$ in the pump-probe arrangement, was used. As-grown and annealed $\mathrm{LT}$ GaAs layers grown at the substrate temperature $T_{\mathrm{s}}=$ $200-400^{\circ} \mathrm{C}$ were used. The carrier lifetime was evaluated as an l'edecay of the measured signal. Two limiting results of the timeresolved reflectivity (normalised), for as-grown LT GaAs grown at $T_{s}=400^{\circ} \mathrm{C}$ and annealed LT GaAs grown at $T_{s}=200^{\circ} \mathrm{C}$, are shown in Fig. 1. The change of the reflectivity in the as-grown sample exhibits two time constants: an initial fast decav and an additional much slower tail. Time constants of 0.2 and $32 \mathrm{ps}$ follow from the fitting of the reflectivity change by two exponentiaj decays. Conversely, the annealed sample shows extremely last reflectivity decay, and a carrier lifetime of $0.20 \mathrm{ps}$ was found. Similar values of $0.17-0.24 \mathrm{ps}$ were obtained on annealed samples prepared at higher substrate temperatures. From this it follows tha for an intrinsically limited PD, i.e. if the RC time constant is smaller than the carrier lifetime, a bandwidth of $\sim 640 \mathrm{GHz}$ might be expected.

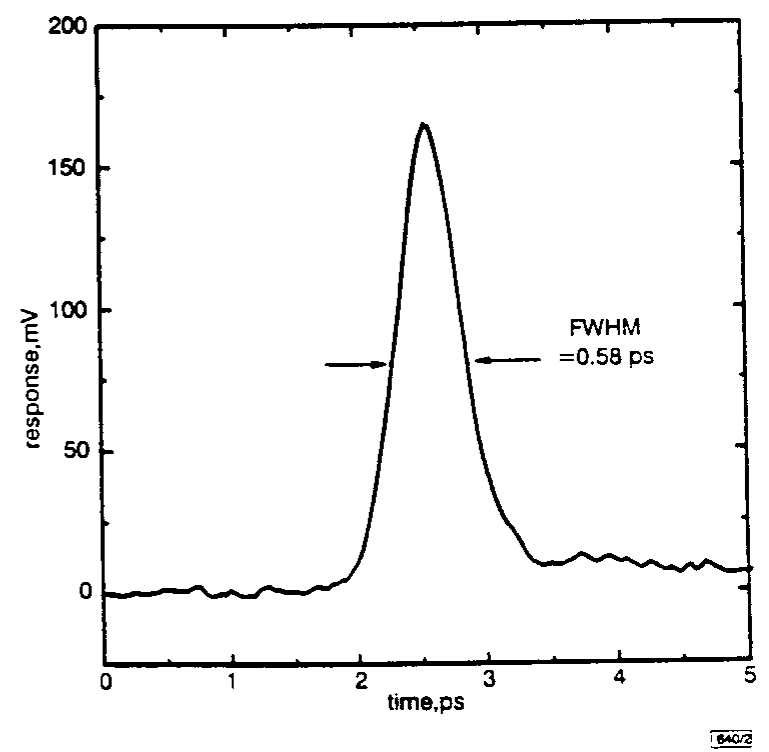

Fig. 2 Time-resolved response of LT GaAs MSM photodetector $\lambda=820 \mathrm{~nm} . T=300 \mathrm{~K}, U_{b}=10 \mathrm{~V}$

Transient behaviour of LT GaAs MSM PDs was studied by measurements of the photoresponse on the femtosecond pump pulse from a sapphire laser $(\hat{\lambda}=820 \mathrm{~nm})$. Fig. 2 shows a typical time-resolved photoresponse measured on devices with an active area of $20 \times 20 \mu^{2}$. interdigitated electrodes with a finger-width it $=1 \mu \mathrm{m}$ and finger-spacing $s=2 \mu \mathrm{m}$. and $10 \mathrm{~V}$ bias voltage. The measured signal was nearly symmetric. i.e. without any tail. and the rise and fall times $(10-90)$ are 0.4 and $0.6 \mathrm{ps}$. respectively. The FWHM is $0.6 \mathrm{ps}$. This is. according to our knowiedge. the fastest response reported on a semiconductor photodetector. Fig. 3 show's

No. 1 


\title{
Absolute thermal lens method to determine fluorescence quantum efficiency and concentration quenching of solids
}

M. L. Baesso and A. C. Bento

Departamento de Física. Universidade Estadual de Maringá, Av. Colombo 5790, 87020-900, Maringá PR, Brazil

A. A. Andrade, J. A. Sampaio, E. Pecoraro, L. A. O. Nunes, and T. Carunda

instituto de Física de Sāo Carlos, Grupo de Espectroscopia de Sólidos, Universidade de São Paulo, Av. Dr. Carlos Botelho 1465, CEP 13560-250, São Carlos SP, Brazil

\author{
S. Gama \\ Instituto de Física Gleb Wataghin, Universidade Estadual de Campinas, 13083-970. Campinas SP, Brazil
}

(Received 1 December 1997)

\begin{abstract}
An absolute thermal lens method to determine fluorescence quantum efficiency and concentration quenching of solids is described in this work. The quantum efficiency of low silica calcium aluminate giasses doped with different concentrations of neodymium dioxide and melted under vacuum conditions to remove water, has been measured by using mode-mismatched thermal lens spectrometry. It has been shown that the thermal lens signal amplitude is lineariy dependent on neodymium concentrations up to $4.0 \mathrm{wt} \%$, changing significantly from 4.5 to $5.0 \mathrm{wt} \%$. indicating that there was quenching of the fluorescence only above $4.0 \mathrm{wt} \%$ neodymium dioxide. The quantitative trearment for the thermal lens effect provided the absolute value of the samples fluorescence quanum efficiency. The technique is simple to perform and can be applied for a wide range of fluorescent materials. [S0163-1829(98)00714-0]
\end{abstract}

PACS number(s): $78.40 . \mathrm{Ha}, 42.65 \mathrm{Jx}, 42.70 . \mathrm{Hj}$

\section{INTRODUCTION}

Fluorescence quantum efficiency $(\phi)$ is one of the most important optical properties of fluorescent materials. The determination of its absolute value, especially for solid samples, has been shown to be difficult. Controversial results in the literatare are due to the limitations of the employed experimental methods. ${ }^{1-7}$ First, pure optical measurements such as the integrating sphere method, demand calibration of the detector. The fluorescent light is polychromatic, therefore introducing inaccuracy in the results. The use of fluorescence lifetime is also inadequate, especially for multilevel energy systems, because it depends on both the value of the nonradiative lifetime and the furorescence decay. These do not obey a single exponential shape. The introduction of photothermal techniques to measure the auantum efficiency in the last decade has brought new perspectives to obtaining more accurate values for this parameter. These methods are based on the determination of the nonradiative quantum efficiency, and are therefore compiementary to the purely optical procedures. ${ }^{124.5}$ A good review on the subject can be found in Ref. 1, which also presents the use of noncontact quadraare photopyroelectric spectroscopy to measure quantum efficiency of Ti:sapphire laser crystals.

In 1978. Brannon and Magde ${ }^{8}$ introduced the thermal lens spectrometry (TLS) to measure fluorescence quanrum efficiency. Since then, the technique has been used to determine this parameter in fuorescent solutions ${ }^{9}$ and fivorescent polymers. ${ }^{10}$ In those experiments, single beam or modematched configuration have been used. and the data were anaiyzed through the parabolic model. However, it has been shown that this model is not realistic. since it cannot predict the rings observed in the far field during the experiments.
Furthermore, the use of mode-mismatched experimental configuration has increased the sensitivity of the technique.

TLS is a noncontacting technique and can be performed in a low-frequency range (time resoived measurements) and in the steady-state mode. In these procedures, the ratio of the absorbed energy that is converted into heat and into fluorescence can be determined. This technique has received special attention in the last few years, particularly its dual-beam mode-mismatched connguration, which has been shown to be very sensitive and inerefore appropriate for measurements in highly transparent materiais. In this arrangement. thi sample is positioned at the waist of the excitation beam, where the power density is maximum, and at the confocal position of the probe beam. By using this arrangement optical absorption coefficient as low as $10^{-7} \mathrm{~cm}^{-1}$ can be measured. The thermal lens effect is created when the excitation laser beam passes through the sample and the absorbed energy is converted into heat, changing the optical path length $s$ and producing a lensiike optical element at the sample. The propagation of the proie beam through the IL will result in either a spreading or a focusing of the beam center, depending on the temperature coefficient of the thermal expansion and electronic polarizability of the sample. "The theoretical model for thermal lens in the mode-mismatched configuration was developed by taking into account the absorbed en. ergy that is converted into heat. This model considers both the aberrant nature of tice thermal lens and the whole change in the optical path lengit induced by the laser beam. It pro. vides a simple analytical expression to interpret the experimental results. This model has been applied successfully to measure quantitativel: the thermooptical parameters of transparent materials. ${ }^{\text {i..12 }}$

Rare-earth doped giasses are interesting for many optical 
applications, including active medium for glass lasers. The fluorescence quantum efficiency of these glasses decreases with the increase in the doping concentration. The fall in its value is highly dependent on the glass composition and structure. For silicate glasses the reduction in the radiatuve quantum efficiency becomes significant over $2 \%$ of $\mathrm{Nd}_{2} \mathrm{O}_{3}$, while in phosphate glasses with $\mathrm{Nd}_{2} \mathrm{O}_{3}$ concentration up to $6 \%$ the decrease in $\phi$ is very small. ${ }^{13}$ Usually the quenching can be observed by monitoring the fiuorescence lifetime ( $\tau$ ) of the sample. A decrease in $\tau$ value would indicate interaction between metai-metal and multiphonon relaxation processes, inducing a reduction in the fluorescence intensity. However, for quantitative measurements this is a limited procedure, since, as mentioned before, the fluorescence decay usually does not obey a single exponential shape.

It is desirable, therefore, to have a quantitarive and accurate method to determine concentration quenching of doped samples without using lifetime measurements.

The effects induced by the introduction of large amounts of rare earth on both the glass structure and the fluorescence quanum efficiency have not yet been investigated in low silica calcium aluminate glasses. These glasses are transparent in the infrared spectral range up to $6 \mu \mathrm{m}$, for samples melted under vacuum counditions. Low silica calcium aluminate glasses are interesting because they car be obtained without using the traditional network former. ${ }^{14-16}$ Their excellent qualities, good chemical durability and high transformation temperatures, allow their use in hostile environments, ${ }^{14}$ such as the cavity of solid-state lasers. The addition of rare-earth oxides to these glasses demands the determination of their quantum efficiency $(\phi)$ for the different doping concentration.

The addition of a rare earth increases the thermal lens effect. The absorbed incident light is converted into heat and fluorescence, and the ratio between the two depends on the temperature induced optical path length change $(d s / d T)$ in the sample. The theoretical trearment for the modemismatched TL effect has been used quantitatively to determine $d s / d T$ of silicate glasses by using an analytical expression for the thermal lens signal which permits evaluation of the fraction of energy that is converted into heat. "The advantage of this method compared with lifetime measurements is that the absorbed energy that is converted into heat is taken into account quantitatively. Furthermore, the method is very sensitive and simple to perform.

The aim of this work is to present the mode-mismatched TL as a quantitative method to determine absolute fluorescence quantum efficiency and the concentration quenching of solids. The experiments have been performed in low silica calcium aluminate glasses prepared with different concentrations of neodymium dioxide. For comparison, the quantum efficiency values were aiso obtained through the Judd-Ofelt model.

\section{EXPERIMENTAL}

The glasses were prepared by melting low silica calcium auminate glasses doped with different concentrations of neodymium dioxide in graphite crucibles. The sample compositions were 41.5 wt $\% \mathrm{Al}_{2} \mathrm{O}_{3}-X, 47.4 \mathrm{wt} \% \mathrm{CaO}, 7 \mathrm{wt} \%$ $\mathrm{SiO}_{2}, 4.1$ wt $\% \mathrm{MgO}$, where $X=0.5,1.0,1.5,2.0,2.5,3.0$,

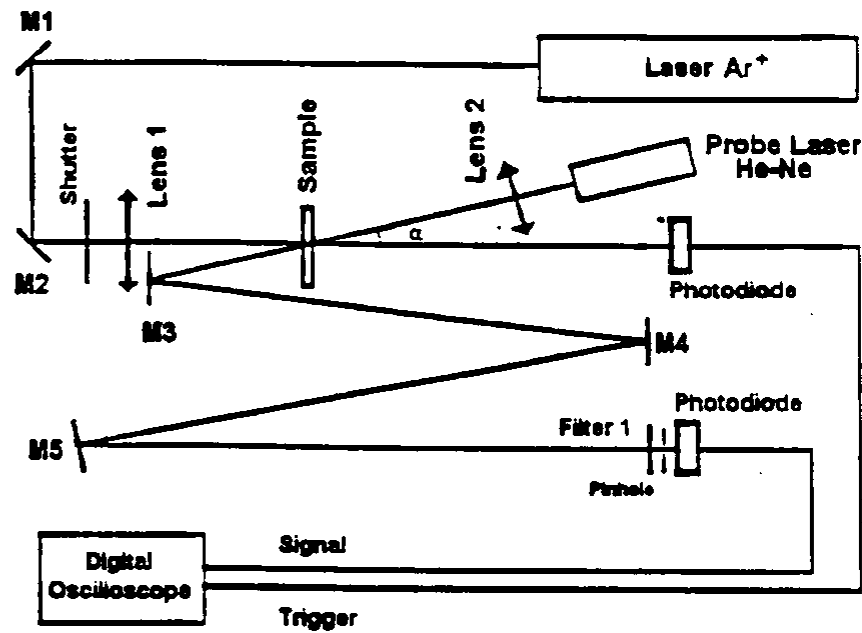

FIG. 1. Mode-mismatched thermal lens experimental setup.

3.5, 4.0, 4.5, and $5.0 \mathrm{wt} \%$ of $\mathrm{Nd}_{2} \mathrm{O}_{3}$. The mixnure was melted under vacrum conditions at $1500^{\circ} \mathrm{C}$. After two hours of meling the heater was switched off and the moved to a cooled chamber close to room temper:samples obtained were cut and polished, resulting in $\mathrm{i}$. approximately $1 \mathrm{~cm}$ in diameter and $3 \mathrm{~mm}$ in thickness. In were analyzed using both $\mathrm{x}$-ray diffraction and optical microscopy in order to investigate their homogeneity. All of them presented good optical quality with a low level of scattering and no evidence of devitrification. The doping concentration for all samples was verified by measuring the optical absorption coefficient in the peak of the absorption band at $590 \mathrm{~nm}$.

The mode-mismatched thermal lens experimental seaup is shown in Fig. 1. The excitation laser used was an argon ion laser (Coherent innova 90 Plus) at $514.5 \mathrm{~nm}$ and a $\mathrm{He}-\mathrm{Ne}$ laser as the probe beam at $632.8 \mathrm{~nm}$. The sample was placed at the waist of the excitation beam and at the confocal position of the probe beam. The probe beam and excitation beam spot sizes at the sample were $236 \mu \mathrm{m}$ and $64.7 \mu \mathrm{m}$. respectively. The experiments were performed by using both time resoived and steady-state methods. In the time resolved procedure the exposure of the sample to the excitation beam was controlled by a shutter. The signal was recorded for about 30 ms during the buildup of the thermai iens. For the steadystate method the thermal lens signal was recorded after $2 \mathrm{~s}$ of illumination. For each sample, the signal was obtained as a function of the excitation laser beam power.

The optical absorption coefficients were determined using the same experimental configuration applied for the TI measurements. The transmitted light of the argon laser at 514.5 nm was recorded for different incident power. The reflections at the two sample surfaces were taken into account.

The fluorescence quanum efficiency value was also obtained by using the Judd-Ofelt model by following the same procedure described in Refs. 17 and 18.

\section{THEORY}

In the aberrant theoretical model for the mode mismatcied TL configuration. the thermal iens effect is treated as an optical path iength change to the probe lase: beam. which can be expressed as an additional prase shift on 
the probe beam wave front after its passing through the sample. The variation of the intensity in the center of the probe beam at the detector caused by the thermal lens can be expressed as ${ }^{11.12}$

$$
\begin{gathered}
I(t)=I(0)\left[1-\frac{\theta}{2}\right. \\
\left.X \operatorname{Lur}^{-1} \cdot *\left(\frac{2 m v}{\left[(1+2 m)^{2}+v^{2}\right] \frac{t_{c}}{2 t}+1+2 m+v^{2}}\right)\right]^{2},
\end{gathered}
$$

where

$$
\begin{gathered}
m=\left(\frac{\omega_{1 p}}{\omega_{e}}\right)^{2} ; \quad v=\frac{Z_{1}}{Z_{c}} \text { when } Z_{c} \gg Z_{2} ; \quad t_{c}=\frac{\omega_{e}^{2}}{4 D} ; \\
\theta=-\frac{P_{e} A_{e} l_{0}}{\sum_{\lambda_{p}}^{\prime}} \frac{d s}{d T} .
\end{gathered}
$$

Here, $t_{c}$ is the characteristic thermal lens time constant, $\omega_{e}$ is the excitation laser beam radius at the sample, $D$ is the sample thermal diffusivity $\left(\mathrm{cm}^{2} / \mathrm{s}\right), K$ is thermal conductivity $\left(J \mathrm{~s} \mathrm{~s}^{-1} \mathrm{~cm}^{-1} \mathrm{~K}^{-1}\right), P_{e}$ is excitation laser beam power (mW), $A_{e}$ is the optical absorption coefficient at the excitation beam wavelength $\left(\mathrm{cm}^{-1}\right), l_{0}$ is the sample thickness (cm), $Z_{c}$ is the confocal distance of the probe beam, $Z_{1}$ is the distance between the probe beam waist and the sample, $Z_{2}$ is the distance between the sample and the detector, $\omega_{1 p}$ is the probe beam radius at the sample, $\theta$ is approximately the phase difference of the probe beam at $r=0$ and $r=2^{1 / 2} \omega_{c}$ induced by the thermal lens, $\lambda_{p}$ is the probe beam wavelength and $I(0)$ is the value of $I(t)$ when the transient time $t$ or $\theta$ is zero.

The above expressions are valid when all the absorbed energy is converted into heat. For fluorescent samples a factor must be introduced in order to take into account the energy emitted. This can be accomplished rewriting the equation for $\theta$ as

$$
\theta=-\frac{P_{e} l_{0}}{K_{\lambda_{p}}} \frac{d s}{d T}\left[A_{0}+\left(A_{D}-A_{0}\right)\left(1-\phi \frac{\lambda_{e}}{\left\langle\lambda_{e m}\right\rangle}\right)\right]
$$

where $\phi$ is the sample radiative quantum efficiency, $\lambda_{e}$ is the excitation beam waveiength and $\left\langle\lambda_{e m}\right\rangle$ is the average wavelength of the fluorescence.

Fron these data the quantum efficiency can be calculated by taking the ratio of Eq. (3) for the doped and undoped samples as follows:

$$
\frac{\theta_{D}}{\theta_{0}}=\frac{A_{0}+\left(A_{D}-A_{0}\right)\left(1-\phi \frac{\lambda_{e}}{\left\langle\lambda_{e m}\right\rangle}\right)}{A_{0}} .
$$

Here, $\theta_{0}$ and $\theta_{D}$ are the probe beam phase shift for the undoped and doped samples, respectively (normalized to the excitation beam power and sample thickness) and $A_{0}$ and $A_{D}$ their optical absorption coefficients. The doped sample ab-

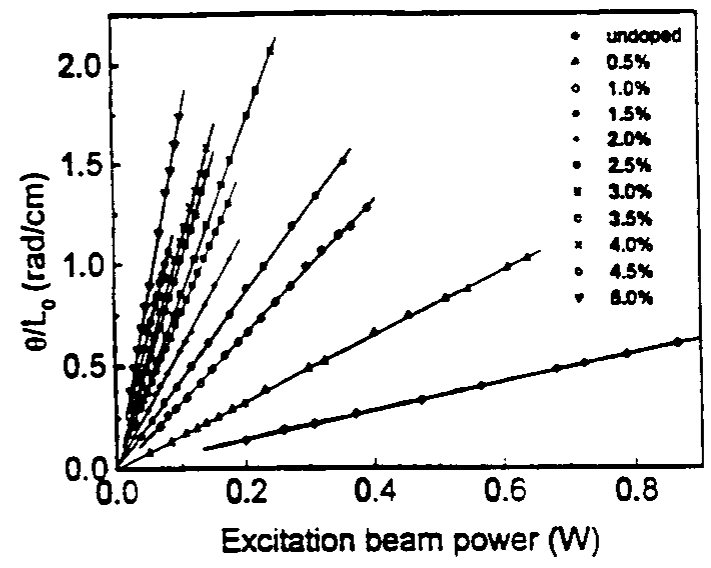

FIG. 2. Normalized thermal lens signal for different excitation laser beam oower at $514.5 \mathrm{~nm}$. The probe beam wavelength is 632.8 $\mathrm{nm}$.

sorption coefficient is considered to be comprised of two contributions coming from the doping and the sample itself, such as

$$
A_{D}=A_{0}+A_{\text {Nd }} \text {. }
$$

where $A_{\mathrm{No}}$ is associated to the neodymium optical absorption coefficient.

Therefore, by measuring the sample optical absorption coefficient and taking the average wavelength of the fluorescence $\left\langle\lambda_{e m}\right\rangle$ for neodymium as approximately $1.054 \mu \mathrm{m}$, the values of the quanum efficiency $\phi$ can be obtained.

\section{RESULTS AND DISCUSSION}

In Fig. 2 the steady-state thermal lens results are shown for different excitation beam power for the samples doped with $\mathrm{Nd}_{2} \mathrm{O}_{3}$. From the linear curve fiting, the values of $\theta$ (normalized to the sample thickness and excitation beam power) were determined for each sampie and represented in Fig. 3. The time resolved measurements provided similar results for the $\theta$ values, as expected. It can be seen that the thermal lens signal increased linearly with neodymium concentration up to $4 \%$ wt $\mathrm{Nd}_{2} \mathrm{O}_{3}$. Above $4 \%$ wt $\mathrm{Nd}_{2} \mathrm{O}_{3}$, the

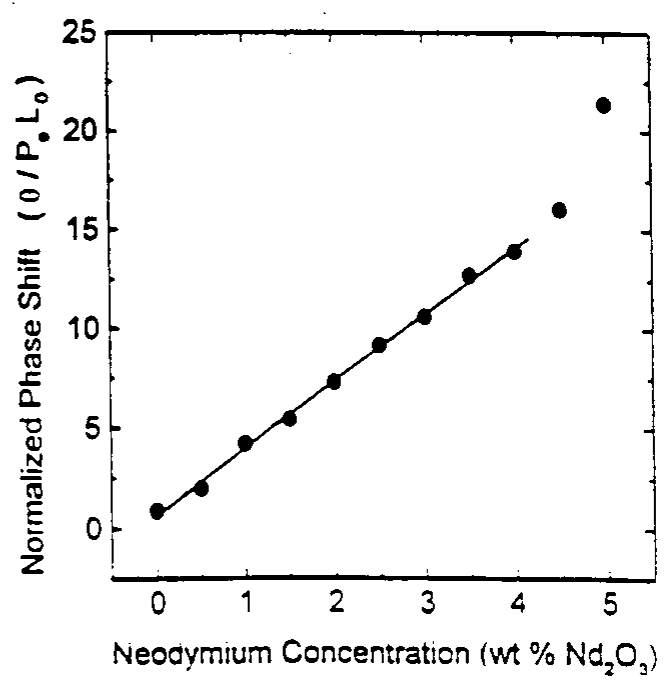

FIG. 3. Normaiized thermal lens signai vs different $\mathrm{Nd}_{2} \mathrm{O}_{3}$ cor: centration in low silica calcium aluminate glasses. 
thermal lens signal increased faster than observed for low doping concentration. This result suggests that the fluorescence rate is constant up to $4 \%$ wt $\mathrm{Nd}_{2} \mathrm{O}_{3}$ and that there was quenching of the fluorescence only for the samples with $4.5 \%$ wt and $5.0 \%$ wt of $\mathrm{Nd}_{2} \mathrm{O}_{3}$.

From these data the quanum efficiency was calculated by performing the linear curve fitting shown in Fig. 3 for the $\theta$ values of the undoped and doped samples with neodymium concentrations up to $4 \%$. Taking the linear angular coefficient, the optical absorption coefficient of the sample and by using Eq. (3), the fluorescence quanaum efficiency was determined to be $0.92 \pm 0.05$. For the samples with $4.5 \%$ wt and $5.0 \%$ wt of $\mathrm{Nd}_{2} \mathrm{O}_{3}$, the values of $\phi$ were $0.86 \pm 0.04$ and $0.67 \pm 0.04$, respectively. The standard deviation of the results was observed to be controlled by the measurements of the optical absorption coefficient, which was about $3 \%$ for all measured samples. The results shown in Figs. 2 and 3 were repeated 5 times and the deviations found for each measurement sequence were always below $1.5 \%$. The standard deviation of $\phi$ values provided by thermal lens in this work are smaller than those accepted in the literature for the JuddOfelt model, which are higher than $10 \% .^{19}$

Uhimann et al. ${ }^{20}$ analyzing four different compositions of calcium aluminate glasses doped with $0.5 \%$ wt of $\mathrm{Nd}_{2} \mathrm{O}_{3}$, have found that the value of the quantum efficiency was around 0.83 , higher than those of silicates, attributing this difference to the lower phonon energy of aluminates. Our results of 0.92 are even higher than those of the authors, and may be associated to the fact that our samples are melted under vacuum conditions, removing the water, and therefore reducing the contribution of the $\mathrm{OH}$ to fluorescence quenching.

In Fig. 4 the values of the quantum efficiency obtained by IL are compared with those determined through the JuddOfelt model in the same samples. If we consider $10 \%$ as the standard deviation of the data obtained through the JuddOfelt model, the results provided by the two methods are in good agreement for doping concentrations up to $2.0 \%$ wt of $\mathrm{Nd}_{2} \mathrm{O}_{3}$. In contrast, for higher doping concentrations, the Judd-Ofelt model, which considers the experimental radiative lifetime, showed quenching of the fuorescence at lower concentrations than those from TL results. It is well known that the irerease in ic:. concentration induess interactions between the pairs of ions, depopulating the ${ }^{4} F_{3 / 2}$ level. The lifetime of this level thus is reduced with a consequent decrease in its emission. The depopulation of the ${ }^{4} F_{3 / 2}$ level has been attributed to the following cross relaxation process: $\left({ }^{4} F_{3 / 2},{ }^{4} I_{9 / 2}\right) \rightarrow\left({ }^{5} I_{15 / 2},{ }^{4} I_{15 / 2}\right)$, followed by a nonradiative decay to ground state, generating heating. ${ }^{21}$ Our results indicate that an additional process, not taken into account by the Judd-Ofelt theory, might be involved. A fraction of those

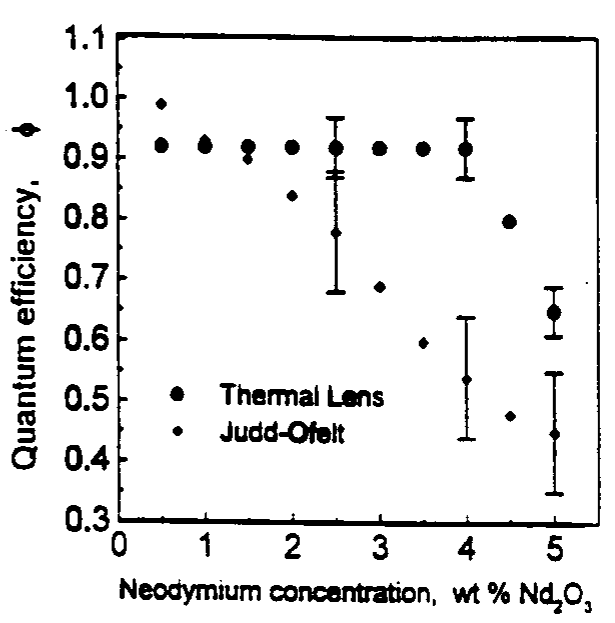

FIG. 4. Fluorescence quantum efficiency vs different $\mathrm{Nd}_{2} \mathrm{O}_{3}$ concentration determined by thermal lens and Judd-Ofelt methods.

photons would populate higher energy levels, with consequent emission in the visible or UV, with no generation of heat or TL signal. The latter mechanism may explain the difference observed between the two methods. The TI method provides therefore the total fiuorescence, while the Judd-Ofelt model determines the emission from the ${ }^{4} F_{3 / 2}$.

In conclusion, our results provided quantitative information about the thermal lens effect in low silica calcium aluminate glasses doped with neodymium. Fluorescence rates appear to be constant for the investigated samples with concentrations up to $4.0 \mathrm{wt} \%$ of $\mathrm{Nd}_{2} \mathrm{O}_{3}$. Quenching of the fluorescence was significant only for the samples doped with $4.5 \%$ wt and $5 \%$ wt of $\mathrm{Nd}_{2} \mathrm{O}_{3}$. The experimental configuration used is very sensitive and we have presented a new method to determine the sample quanum efficiency :therefore, to evaluate the fraction of the radiative 2 : diative processes in these glasses. The method $n_{2}$. shown to be useful to investigate concentration effects on neodymium fluorescence. The technique is simple to perform, is noncontacting and its high sensitivity indicates that it can be applied for a wide range of fluorescent materials. Furthermore, our results have shown that by melting the glasses under vacuum conditions the fuorescence quantum efficiency ot the doping is increased, indicatung that calcium aluminate glasses are strong candidates to be used as an active medium for solid-state lasers.

\section{ACKNOWLEDGMENTS}

We are thankful to the Brazilian National Council (CNPq and to FAPESP for the financial support of this work.
${ }^{1}$ A. Mandelis, J. Vanniasinikan, and S. Budhudu, Phys. Rev. B 48, 6808 (1993).

'A. Rosencwaig and E. A. Hildum, Pnys. Rev. B 23, 3301 (1981).

${ }^{3}$ I. M. Tomas, S. A. Payne. and G. D. Wilke. J. Non-Cryst. Solids 151, 183 (1992).

${ }^{4}$ R. S. Quimby and W. M. Yen, Opt. Lett. 3, 181 (1978).
${ }^{5}$ R. C. Powell, D. P. Neikirk, and D. Sariar, J. Opt. 486 (1980).

${ }^{6}$ A. J. Ramponi and J. A. Caird. J. Appl. Phys. 63, 547t

'E. M. Dianor, A. Ya. Karasik, V. B. Neustruev, Prokhorov, and I. A. Shemerbakov, Sov. Phys. Dok!. 20, (1976). 
${ }^{8}$ J. H. Brannon and D. Magcie, J. Phys. Chem. 82, 705 (1979)

${ }^{9}$ J. Shen and R. D. Snook. Chem. Phys. Lett. 155, 583 (1989)

${ }^{10} \mathrm{M}$. L. Lesiecki and I. M. Drake, Appl. Opt. 21. 557 (1982).

${ }^{11}$ M. L. Baesso, J. Shen, and R. D. Snook. J. Appl. Phys. 75, 3232 (1994).

${ }^{12}$ J. Shen, R. D. Lowe, and R. D. Snook. Chem. Phys. 165, 385 (1992).

${ }^{13}$ O. Deutschbein, M. Faulstich, W. Jahn, G. Krolla, and N. Neuroth. Appl. Opt. 17. 2228 (1978).

${ }^{14} \mathrm{~J}$. E. Shelby, J. Am. Ceram. Soc. 68, 155 (1985).
${ }^{15}$ W. A. King and J. E. Shelby. Phys. Chem. Glasses 37. 1 (1996)

${ }^{16}$ J. R. Dawy, Glass Technol. 19, 32 (1978)

${ }^{17}$ B. R. Judd, Phys. Rev. 127, 750 (1962).

${ }^{18}$ G. S. Felt J. Chem. Phys. 37, 511 (1962).

${ }^{19}$ M. J. F. Digonnet. Rare Earth Doped Fiber Lasers and Amplifiers (Marcel Dekker. New York, 1993).

${ }^{20}$ E. V. Uhimann, M. C. Weinberg. N. J. Kreidl, L. L. Burgner, R. Zanoni, and K. H. Church, J. Non-Cryst. Solids 178, 15 (1994).

${ }^{21}$ H. G. Danielmeyer, M. Blatre, and P. Balmer, Appl. Phys. 1. 269 (1973). 


\title{
Neodymium concentration dependence of thermo-optical properties in low silica calcium aluminate glasses
}

\author{
M.L. Baesso ${ }^{\text {a. }}$, A.C. Bento ${ }^{a}$, A.A. Andrade ${ }^{b}$, T. Catunda ${ }^{\text {b }}$, J.A. Sampaio ${ }^{c}$. \\ S. Gama ${ }^{c}$ \\ ¿ Departamento de Física. Unicersidade Estadual de Maringái. Al. Colombo 5790. 87020-400. Maringá PR. Bra-il \\ "Instinuo de Fisica de São Carlos. Grupo de Espectroscopia de Sólidos. Unitersidade de São Paulo. Al. Dr. Carlos Botelho 1465. \\ CEP 13560-250. Sãil Carlos / SP. Bra-il \\ "Intituto de Fisica Gleb Wataghin. Unitersidade Estadual de Campinas. Unicamp. 13083-970. Campinas SP. Bra-il
}

\begin{abstract}
Thermal lens spectrometry of thermo-optical properties of low silica calcium aluminate glasses doped with different concentration of neodymium dioxide was conducted. Thermal lens signal amplitude presented a linear dependence for neodymium concentrations up to $2.0 \mathrm{wt} \%$. indicating that there was no fluorescence quenching. The quantitative treatment for the thermal lens effect also allowed determination of the absolute value of quantum efficiency. 0.87 . for all samples. Thermal diffusivity is around $5.5 \times 10^{-3} \mathrm{~cm}^{2} / \mathrm{s}$ with some dependence on Nd concentration. 1997 Elsevier Science B.V.
\end{abstract}

\section{Introduction}

Low silica calcium aluminate glasses are transparent in the infrared (IR) to $5 \mu \mathrm{m}$. Several matrix compositions and preparing conditions have been developed in order to understand their optical and structural properties $[1-7]$. These glasses are interesting not only because of their good chemical durability and high transformation temperatures. but also because they can be obtained without using the traditional network former [3.6]. It has been shown that the introduction of small amounts of silica and magnesium oxide (or barium oxide) in binary calcium aluminate glass improves the glass forming region in the phase diagram without significant IR transmission [2] loss. Transmission in the infrared can also be

\footnotetext{
Corresponding author. Fax: +55-16 272 2218: e-mail: tomaz (ঐifsc.sc.usp.br.
}

affected by water which can be avoided by melting under vacuum conditions. With this procedure Davy [2] has achieved IR transmission as high as that of sapphirc.

It has been shown that addition of rare earth oxides to glasses induces structural changes. modifying thermal expansion coefficient. density and moiar volume [8-10]. Rare earth oxides can act either as a network formers for fluorozirconate glasses or as modifiers for others known tluorides and oxides glasses which contain smaller network formers [8$10]$. In the latter they induce fluorescence broadening. These rare earth doped glasses are interesting for many optical applications. including active media glass lasers. It is desirable. therefore. to introduce large amounts of rare earth and investigate the changes in the glass structure and properties. The great limitations of working close to the limit of the phase diagram. as in the case of low silica calcium 
aluminate glasses, is that the introduction of a small amount of rare earth in the glass composition introduces devitrification and, therefore, significant changes in the glass.

Another problem to consider is that the quantum efficiency decreases with increase in the doping concentration. For phosphate glasses with $\mathrm{Nd}_{2} \mathrm{O}_{3}$ concentration up to $6 \%$ reduction in radiative quantum efficiency is small [11]. Usually the quenching can be observed by monitoring the fluorescence lifetime of the sample. A fall in its value would indicate interaction between metal-metal and multiphonon relaxation processes, inducing reduction in the fluorescence intensity. The introduction of rare earth is therefore controlled by both the limit of vitrification and fluorescence [11-13] quenching.

Addition of rare earth also increases the thermal lens effect (TL) when the glass is illuminated by a laser beam. Absorbed incident light is converted into heat and fluorescence. and the ratio between the two depends on the temperature induced optical path length change $(\mathrm{d} s / \mathrm{d} T)$ in the sample. Recently, the TL effect has been used in a quantitative way to determine $\mathrm{d} s / \mathrm{d} T$ of silicate glasses by using an analytical expression for the TL signal which permits evaluation of the fraction of energy which is converted into heat [14]. The advantage of this method compared with lifetime measurement is that the absorbed energy is taken into account quantitatively.

Neodymium is the most studied rare earth element in glasses and its properties are well known for several glass compositions. In this works $41.5 \mathrm{wt} \%$ $\mathrm{Al}_{2} \mathrm{O}_{3}, 47.4 \mathrm{wt} \% \mathrm{CaO} .7 \mathrm{wt} \% \mathrm{SiO}_{2}, 4.1 \mathrm{wt} \% \mathrm{MgO}$ glasses were prepared with neodymium dioxide $(0.5$ wt $\%, 1.0 \mathrm{wt} \%, 1.5 \mathrm{wt} \%$ and $2 \mathrm{wt} \%)$ under vacuum melting conditions. Since this composition stands close to the edge of the phase diagram. our interest is to investigate the ratio between fluorescence and thermal heating of the samples as a function of doping concentration. To do so the mode mismatched thermal lens spectrometry was employed. In addition. a new method to determine the quantum efficiency will be presented.

\section{Experimental}

Glasses were prepared by melting low silica calcium aluminate glasses with neodymium dioxide of
$0.5 \mathrm{wt} \%, 1.0 \mathrm{wt} \%, 1.5 \mathrm{wt} \%$ and $2 \mathrm{wt} \%$ of $\mathrm{Nd}_{2} \mathrm{O}_{3}$ in graphite crucibles. The basic sample composition was $41.5 \mathrm{wt} \% \mathrm{Al}_{2} \mathrm{O}_{3}, 47.4 \mathrm{wt} \% \mathrm{CaO} .7 \mathrm{wt} \% \mathrm{SiO}_{2}$, $4.1 \mathrm{wt} \% \mathrm{MgO}$. This concentration range $(0.5-2 \mathrm{wt} \%)$ is equivalent to $(0.65-2.6) \times 10^{20} \mathrm{Nd}^{3+} / \mathrm{cm}^{3}$. The mixture was melted under vacuum conditions at $1500^{\circ} \mathrm{C}$. After $2 \mathrm{~h}$ of melting the heater was switched off and the crucible moved to a cooled chamber near room temperature. Samples obtained were cut and polished into $1 \mathrm{~cm}$ diameter and around $3 \mathrm{~mm}$ thick disks and analyzed using both $\mathrm{X}$-ray diffraction and a $\mathrm{He}-\mathrm{Ne}$ laser in order to investigate their homogeneity. They presented good optical quality with low scattering and no observed devitrification.

In the mode mismatched thermal lens measurement the sample is illuminated by a $\mathrm{TM}_{00}$ Gaussian laser beam (excitation beam). and a temperature rise is produced by non-radiative decay processes following the optical energy absorption. The change in refractive index with temperature produces a lens like optical element at the sample. called thermal lens. Propagation of a weak probe beam through the thermal lens will be affected. resulting in a variation of its intensity at the beam center in the far field. By measuring these changes. information of the thermo-optical properties of the samples can be obtained. In the theoretical model for the mode mismatched configuration. the variation of intensity in the center of the probe beam caused by the thermal lens can be expressed as [14.15]

I(t)

$$
\begin{aligned}
= & l(0) \\
& \times\left\{1-\frac{\theta}{2} \operatorname{atan}\left[\frac{2 m v^{\prime}}{\left[(1+2 m)^{2}-v^{2}\right] t+2 t+1+2 m+v^{2}}\right]\right\}^{2} .
\end{aligned}
$$

where

$$
\begin{aligned}
& m=\left(\frac{\omega_{1 \mathrm{p}}}{\omega_{\mathrm{c}}}\right)^{2}: \quad V=\frac{Z_{\mathrm{l}}}{Z_{\mathrm{c}}} \text { when } Z_{i} \gg Z_{i}: \\
& t_{\mathrm{c}}=\frac{\omega_{i}^{2}}{4 D}: \quad \theta=-\frac{P_{\mathrm{e}} A_{\mathrm{c}} l_{\mathrm{n}}}{K \lambda_{\mathrm{p}}} \frac{\mathrm{d} s}{\mathrm{~d} T} .
\end{aligned}
$$

Here. $t_{c}$ is the characteristic thermal lens time constant. $\omega_{\mathrm{e}}$ excitation laser beam radius at the sample. 
$D$ is the sample thermal diffusivity $\left(\mathrm{cm}^{2} / \mathrm{s}\right), K$ is thermal conductivity $\left(\mathrm{J} \mathrm{s}{ }^{-1} \mathrm{~cm}^{-1} \mathrm{~K}^{-1}\right), P_{\mathrm{c}}$ is excitation laser beam power $(W), A_{e}$ is the optical absorption coefficient at the excitation beam wavelength $\left(\mathrm{cm}^{-1}\right), l_{0}$ is the sample thickness $(\mathrm{cm}), Z_{\mathrm{c}}$ is the confocal distance of the probe beam. $Z_{1}$ is the distance between probe beam waist and sample, $Z_{2}$ is the distance between the sample and the detector. $\omega_{1 \mathrm{p}}$ is probe beam radius at the saniple. $\theta$ is approximately the phase difference of the probe beam at $r=0$ and $r=2^{1 / 2} \omega_{\mathrm{e}}$ induced by the thermal lens. $\lambda_{\mathrm{p}}$ is the probe beam wavelength and $I(0)$ is the value of $I(t)$ when the transient time. $t$. or $\theta$ is zero.

The above expressions are valid when all absorbed energy is converted into heat. For fluorescent samples. a factor should be introduced in order to take into account the energy emitted. For a doped sample we consider that $A_{\mathrm{NJ}}=A_{\mathrm{D}}-A_{0}$ is the ion contribution to the absorption. where $A_{0}$ and $A_{0}$ are the absorption coefficients at $\lambda_{c}$ of the doped and undoped samples respectively. In this case the expression for $\theta$ in Eq. (2) should be rewritten as

$$
\begin{aligned}
\theta= & -\frac{P_{\mathrm{e}} l_{t 1}}{K \lambda_{\mathrm{r}}} \frac{\mathrm{d} s}{\mathrm{~d} T} \\
& \times\left[A_{11}+\left(A_{\mathrm{D}}-A_{01}\right)\left(1-\phi \frac{\lambda_{\mathrm{c}}}{\left\langle\lambda_{\mathrm{cm}}\right\rangle}\right)\right] .
\end{aligned}
$$

where $\phi$ is the sample radiative quantum efficiency. $\lambda_{c}$ is the excitation beam wavelength and $\left\langle\lambda_{\mathrm{cm}}\right\rangle$ is the average wavelength of the fluorescence. In the case of the $\mathrm{Nd}^{-3}$ ion. when a green photon $\left(\lambda_{c}=\right.$ $514.5 \mathrm{~nm}$ or $\left.-1.94 \times 10^{+} \mathrm{cm}^{-1}\right)$ is absorbed. the system rapidly decays to the metastable ${ }^{+} F_{3,2}$ level (at $-1.1 \times 10^{-4} \mathrm{~cm}^{-1}$ ). The ${ }^{4} F_{3 / 3}$ level can decay radiatively to the states: ${ }^{4} I_{5}, .{ }^{+} I_{3}, .{ }^{4} I_{11}$, and ${ }^{t} I_{9}$ : , with emission at $1880,1350.1060$ and 880 $\mathrm{nm}$. respectively. It is also possible nonradiative decay ${ }^{+} F_{3,2} \rightarrow{ }^{+} I_{15: 2}$ (with the emission of phonons). In this work the fluorescent quantum efficiency $\phi$ is the total radiative probability from ${ }^{4} F_{3}$,

Apparatus for mode mismatched thermal lens measurements is shown elsewhere [14.15]. The excitation laser used was an argon ion laser at $514.5 \mathrm{~nm}$ and a $\mathrm{He}-\mathrm{Ne}$ laser as the probe beam at $632.8 \mathrm{~nm}$. The sample was placed at the waist of the excitation beam and at the contocal position of the probe beam.
The probe beam and excitation beam spot sizes at the sample were $236 \mu \mathrm{m}$ and $61 \mu \mathrm{m}$ respectively. Exposure of the sample to the excitation beam was controlled by means of a shutter. The signal was recorded for about $100 \mathrm{~ms}$ during the build up of the thermal lens.

Optical absorption spectra were determined by using a Perkin-Elmer Lambda-9 Spectrophotometer. The absorption coefficients $A_{0}$ and $A_{\mathrm{D}}$, at $\lambda_{\mathrm{e}}=$ $514.5 \mathrm{~nm}$. were measured with the $\mathrm{Ar}^{+}$laser. The reflections in the two surfaces were taken into account. The lifetime measurements was performed following the same procedure as described by Snoeks et al. [16.17]. Here. an Argon laser was used at 514.5 $\mathrm{nm}$ and the fluorescent signal was recorded by using a fast photodiode.

\section{Results}

In Table 1 the values of all parameters obtained in this work are presented. Fig. I shows a normalized time resolved thermal lens signal for the $0.5 \mathrm{wt}$ \% $\mathrm{Nd}_{2} \mathrm{O}_{3}$ doped sample. where the excitation beam power is $610 \mathrm{~mW}$. From the curve fitting using $\mathrm{Eq}$. (1) we found $H=-(0.239 \pm 0.002)$ and $t_{c}=(1.68$ $\pm 0.01) \mathrm{ms}$. Lising this $t_{\mathrm{s}}$ and Eq. (2) we calculated the thermal diffusivity $D=5.5 \times 10^{-3} \mathrm{~cm}^{2} / \mathrm{s}$ and the same procedure was used to obtain the $D$ values presented in Table 1. The thermal diffusivity was

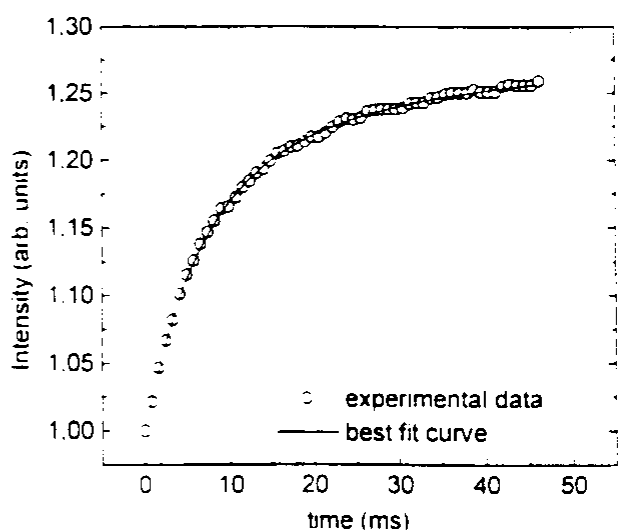

Fig. 1. Time resolsed experimental data and their hest fill curse for the $0.5 \mathrm{ml} ; \mathrm{Vid}, \mathrm{O}$. Joped alnnice. The power of excitation heam is $610 \mathrm{mlll}$ at $51+5$ nm: the prohe beam is at $6.32 .8 \mathrm{~nm}$. 
Table 1

Thermo-optical experimental parameters of the investigated giasses: $A$. absorption coefficient at $\lambda_{e}=515 \mathrm{~nm}$. thermal diffusivity $D$ and fluorescence lifetime $\tau$

\begin{tabular}{llll}
\hline Sample & $\begin{array}{l}\text { Absorption coefficient } \\
\left(\mathrm{cm}^{-1}\right)\end{array}$ & $\begin{array}{l}D \\
\left(\mathrm{~cm}^{2} / \mathrm{s}\right)\end{array}$ & $\begin{array}{l}\tau \\
(\mu \mathrm{s})\end{array}$ \\
\hline Undoped & $0.073 \pm 0.003$ & $5.5 \pm 0.05$ & \\
$0.5 \mathrm{wt} \% \mathrm{Nd}_{2} \mathrm{O}_{3}$ & $0.19 \pm 0.01$ & $5.6 \pm 0.05$ & $325 \pm 6$ \\
$1.0 \mathrm{wt} \% \mathrm{Nd}_{2} \mathrm{O}_{2}$ & $0.57 \pm 0.01$ & $5.8 \pm 0.05$ & $317 \pm 6$ \\
$1.5 \mathrm{wt} \% \mathrm{Nd}_{2} \mathrm{O}_{3}$ & $0.78 \pm 0.01$ & $6.0 \pm 0.05$ & $293 \pm 6$ \\
$2.0 \mathrm{wt} \% \mathrm{Nd}_{2} \mathrm{O}_{3}$ & $0.96 \pm 0.01$ & $5.5 \pm 0.03$ & $279 \pm 6$ \\
\hline
\end{tabular}

shown to increase with the concentration up to 1.5 wt $\%$, from $5.5 \times 10^{-3} \mathrm{~cm}^{2} / \mathrm{s}$ for the uriloped sample. to $6.0 \times 10^{-3} \mathrm{~cm}^{2} / \mathrm{s}$ for $1.5 \mathrm{wt} \%$ of $\mathrm{Nd}_{2} \mathrm{O}_{3}$. However the sample with $2 \mathrm{wt} \%$ presented smaller diffusivity $D=5.5 \times 10^{-3} \mathrm{~cm}^{2} / \mathrm{s}$.

Measurements of the fluorescence lifetime showed small fluorescence quenching effects in the concentration range studied: $\tau$ varies between $325 \mu$ s $(0.5$ $\mathrm{wt} \%)$ to $279 \mu \mathrm{s}(2 \mathrm{wt} \%)$.

The quantum efficiency can be calculated by taking the ratio of Eq. (3) for the doped and undoped sampies as follows:

$\frac{\theta_{\mathrm{D}}}{\theta_{0}}=1+\frac{\left(A_{\mathrm{D}}-A_{03}\right)}{A_{0}}\left(1-\phi \frac{\lambda_{\mathrm{c}}}{\left\langle\lambda_{\mathrm{cm}}\right\rangle}\right)$.

Here. $\theta_{0}$ and $\theta_{\mathrm{D}}$ are the probe beam phase shift for the undoped and doped samples respectively (normalized with respect to the excitation beam power

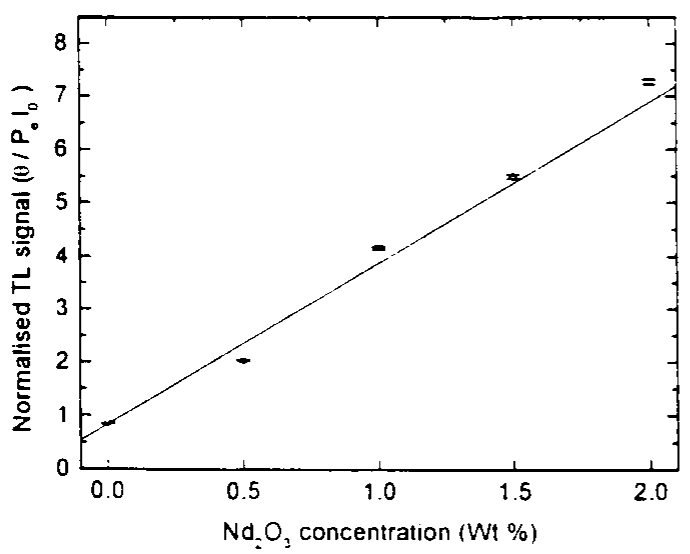

Fig. 2. Normalized thermat signal versus different $\mathrm{Nd}_{2} \mathrm{O}$, concentration in low silica calcium aluminate glasses. and sample thickness), $\lambda_{\mathrm{e}}=515 \mathrm{~nm}$ and $\left\langle\lambda_{\mathrm{em}}\right\rangle \sim$ $1.01 \mathrm{~nm}$. In Fig. 2, the results normalized to the sample thickness and laser beam power $\left(\theta / P_{e} l_{0}\right)$ are shown for different samples. It can be observed that the thermal lens effect increases linearly with neodymium concentrations. The linear coefficient obtained in the fit of Fig. 2 has an uncertainty of $\sim 4 \%$ indicating a good linear behavior. Once $\left(A_{D}\right.$ $-A_{0}$ ) increases linearly with the concentration. from Eq. (4) the behavior on Fig. 2 suggests that. in this concentration range. the quantum efficiency is approximately constant and $\phi=(0.87 \pm 0.1)$.

\section{Discussion}

The quantitative result obtained by the TL technique shows a direct way to investigate the ratio between radiative and nonradiative processes in neodymium doped low silica calcium aluminate glasses. It is a new method to investigate quantum efficiency in glasses and has been shown to be useful for investigations of concentration effects in doped fluorescent glasses. The source of errors in this method are related to the determination of the optical absorption coefficient through conventional measurement. which can introduce some deviations in the results. Here an approximation was made considering that the ratio between $\mathrm{d} s / \mathrm{d} T$ and thermal conductivity remained constant for the different samples. This can be done because the parameter $\mathrm{d} s / \mathrm{d} T$ is associated with the probe wavelength. i.e.. $632.8 \mathrm{~nm}$. where the absorption coefficient does not present significant changes for the different samples investigated. For studies using the probe beam wavelength in the absorption bands. this is not valid since the value for this parameter can change drastically for doped samples [14]

The result $\phi=(0.87 \pm 0.1)$ is in reasonable agreement with $\phi$ between 0.75 to 0.83 calculated using Judd-Ofeit model for similar glasses [18]. and with $\phi \sim(0.9 \pm 0.08)$ measured in silicate glasses with photoacoustics [19].

\section{Conclusions}

Results provided quantitative information about thermal lens effect in low silica calcium aluminate 
glasses doped with neodymium. The experimental configuration used is very sensitive and we have presented a new method to determine the sample quantum efficiency $\phi$ and. therefore, to evaluate the fraction of the radiative and nonradiative processes in these glasses. Fluorescence rates presented small quenching effect up to $2.0 \mathrm{wt} \%$ of $\mathrm{Nd}_{2} \mathrm{O}_{3}$, in good agreement with the constant $\phi$ obtained by thermal lens. We also determined the thermal diffusivity of these glasses. The method appears to be useful to investigate concentration effects on neodymium fluorescence.

\section{Acknowledgements}

We are thankful to the Brazilian National Council $(\mathrm{CNPq})$ and to FAPESP for the financial support of this work.

\section{References}

[1] H.C. Halner. N.J. Kreidl. R.A. Weidel. J. Am. Ceram. Soc $+1(1958) 315$
[2] J.R. Dawy. Glass Technol. 19 (1978) 32

[3] J.E. Shelby. J. Am. Ceram. Soc. 68 (1985) 155

[4] P.L. Higby, R.J. Ginther. I.D. Aggarwal. E.J. Friebeie. J. Non-Cryst. Solids $126(1990) 209$

[5] D.A. Dutt. P.L. Higby. D.L. Griscom. Phys. Chem. Glasse 33 (1992) 51

[6] W.A. King. J.E. Shelby. Phys. Chem. Glasses 37 (1996)

[7] C. Oprea. D. Togan. C. Popescu. Thermochim. Acta 194 (1992) 165.

[8] L. Lucas. M. Chanthanasinh. M. Poulain. M. Pouiain. P. Brun. M.J. Weber. J. Non-Cryst. Solids 27 (1978) 273.

[9] J.T. Kohli. J.E. Shelby. Phys. Chem. Giasses 32 (1991) 67

[10] J.E. Shelby. J.T. Kohli. J. Am. Ceram. Soc. 73 (1990) 39

[11] O. Deutschbein. M. Faulstich. W. Jahn. G. Krolla. .N. Neu roth. Appl. Opt. 17 (1978) 2228.

[12] J. Fernandes. R. Balda. M.A. Arriandiaga. Opt. Mater. + (1994) 91.

[13] H. Ebendorff-Heidepriem. W. Seeber. D. Ehrt. J. Non-Cryst Solids 183 (1995) 191

[14] M.L. Baesso. J. Shen. R.D. Snook. J. Appl. Phys. 75 (1994) 3232.

[15] J. Shen. R.D. Lowe. R.D. Snook. Chem. Phys. 165 (1992) 385.

[16] E. Snoeks. P.G. Kik. A. Polman. Opt. Mater. 5 (199h) 159

[17] W. Koechner. Solid State Laser Engineering. th Ed. (Springer. Berlin. 1996) p. 55

[18] E.V. Uhimann. M.C. Weinberg. N.J. Kreidel. L.L. Burgner R. Zanoni. K.H. Church. J. Non-Cryst Solids $178(199+)$ is

[19] A. Rosencwaig. E.A. Hildum. Phys. Rev. B23 (1981) 3301 